Research Article

\title{
On a Partial q-Analog of a Singularly Perturbed Problem with Fuchsian and Irregular Time Singularities
}

\author{
Stephane Malek ii \\ University of Lille, Laboratoire Paul Painlevé, 59655 Villeneuve d'Ascq cedex, France \\ Correspondence should be addressed to Stephane Malek; stephane.malek@univ-lille.fr
}

Received 28 May 2019; Accepted 19 October 2019; Published 10 January 2020

Academic Editor: Luigi Rodino

Copyright (C) 2020 Stephane Malek. This is an open access article distributed under the Creative Commons Attribution License, which permits unrestricted use, distribution, and reproduction in any medium, provided the original work is properly cited.

A family of linear singularly perturbed difference differential equations is examined. These equations stand for an analog of singularly perturbed PDEs with irregular and Fuchsian singularities in the complex domain recently investigated by A. Lastra and the author. A finite set of sectorial holomorphic solutions is constructed by means of an enhanced version of a classical multisummability procedure due to $\mathrm{W}$. Balser. These functions share a common asymptotic expansion in the perturbation parameter, which is shown to carry a double scale structure, which pairs $q$-Gevrey and Gevrey bounds.

\section{Introduction}

In this work, we focus on singularly perturbed linear partial $q-$ difference differential equations which couple two categories of operators acting both on the time variable, so-called $q$ difference operators of irregular type and Fuchsian differential operators. As a seminal reference concerning analytic and algebraic aspects of $q$-difference equations with irregular type, refer [1], and for a far reaching investigation of Fuchsian ordinary and partial differential equations, refer [2].

Our equations are presented in the following manner

$$
\begin{aligned}
Q\left(\partial_{z}\right) u(t, z, \epsilon)= & R_{D}\left(\partial_{z}\right) \epsilon^{\delta_{D} k m_{D}}\left(t^{\delta_{D} k} \sigma_{q ; t}^{\delta_{D}} t \partial_{t}\right)^{m_{D}} u(t, z, \epsilon) \\
& +P\left(t, z, \epsilon, t^{k} \sigma_{q ; t}, t \partial_{t}, \partial_{z}\right) u(t, z, \epsilon) \\
& +f(t, z, \epsilon),
\end{aligned}
$$

for vanishing initial data $u(0, z, \epsilon) \equiv 0$, where $k, \delta_{D}, m_{D} \geq 1$ are integers, $\sigma_{q ; t}$ represents the dilation map $t \longrightarrow q t$ acting on time $t$ for some real number $q>1$, and $Q(X), R_{D}(X)$ stand for polynomials in $\mathbb{C}[X]$. The main block $P(t, z, \epsilon$, $\left.V_{1}, V_{2}, V_{3}\right)$ is polynomial in the arguments $t, V_{1}, V_{2}, V_{3}$, holomorphic in the perturbation parameter $\epsilon$ on a disc
$D\left(0, \epsilon_{0}\right) \subset \mathbb{C}$ centered at 0 and in the space variable $z$ on a horizontal strip of the form $H_{\beta}=\{z \in \mathbb{C} /|\operatorname{Im}(z)|<\beta\}$, for some $\beta>0$. The forcing term $f(t, z, \epsilon)$ is analytic relatively to $(z, \epsilon) \in H_{\beta} \times D\left(0, \epsilon_{0}\right)$ and defines an entire function w.r.t $t$ in $\mathbb{C}$ with (at most) $q$-exponential growth (see (60), for precise bounds).

This paper is a natural continuation of the study [3] by Lastra and Malek and will share the same spine structure. Indeed, in [3], we aimed attention at the next problem

$$
\begin{aligned}
Q\left(\partial_{z}\right) y(t, z, \epsilon)= & R_{D}\left(\partial_{z}\right) \epsilon^{k \delta_{D}}\left(t^{k+1} \partial_{t}\right)^{\delta_{D}}\left(t \partial_{t}\right)^{m_{D}} y(t, z, \epsilon) \\
& +H\left(z, \epsilon, t^{k+1} \partial_{t}, t \partial_{t}, \partial_{z}\right) y(t, z, \epsilon) \\
& +h(t, z, \epsilon),
\end{aligned}
$$

for vanishing initial data $y(0, z, \epsilon) \equiv 0$, where $Q(X), R_{D}(X)$, $H\left(z, \epsilon, V_{1}, V_{2}, V_{3}\right)$ stand for polynomials in their arguments $X, V_{1}, V_{2}, V_{3}$ as above and $h(t, z, \epsilon)$ is like the forcing term $f(t, z, \epsilon)$ but with (at most) exponential growth in $t$. Under convenient conditions put on the shape of (2), we are able to construct a set of genuine bounded holomorphic solutions expressed as a Laplace transform of order $k$ along a halfline $L_{\gamma_{p}}=\mathbb{R}_{+} \exp \left(\sqrt{-1} \gamma_{p}\right)$ and Fourier inverse integral in space $z$ : 


$$
\begin{aligned}
y_{p}(t, z, \epsilon)= & \frac{k}{(2 \pi)^{1 / 2}} \int_{-\infty}^{+\infty} \int_{L_{\gamma_{p}}} V_{p}(\tau, m, \epsilon) \\
& \cdot \exp \left(-\left(\frac{\tau}{\epsilon t}\right)^{k}\right) e^{i z m} \frac{\mathrm{d} \tau}{\tau} \mathrm{d} m,
\end{aligned}
$$

where the Borel/Fourier map $V_{p}(\tau, m, \epsilon)$ is itself set forth as a Laplace transform of order $k^{\prime}=k \delta_{D} / m_{D}$ :

$$
V_{p}(\tau, m, \epsilon)=k^{\prime} \int_{L_{\gamma_{p}}} W_{p}(u, m, \epsilon) \exp \left(-\left(\frac{u}{\tau}\right)^{k^{\prime}}\right) \frac{\mathrm{d} u}{u},
$$

where $W_{p}(u, m, \epsilon)$ has (at most) exponential growth along $L_{\gamma_{p}}$ and exponential decay in phase $m$ on $\mathbb{R}$. The resulting maps $y_{p}(t, z, \epsilon)$ are therefore expressed as iterated Laplace transforms following a so-called multisummability procedure introduced by Balser, see [4]. These functions define bounded holomorphic functions on domains $\mathscr{T} \times H_{\beta} \times \mathscr{E}_{p}$ for a well-selected bounded sector $\mathscr{T}$ at 0 and where $\underline{\mathscr{E}}=$ $\left\{\mathscr{E}_{p}\right\}_{0 \leq p \leq \varsigma^{-1}}$ is a set of sectors which covers a full neighborhood of 0 and is called a good covering (cf. Definition 6). Additionally, the partial maps $\epsilon \longmapsto y_{p}(t, z, \epsilon)$ share on $\mathscr{E}_{p}$ a common asymptotic expansion $\hat{y}(t, z, \epsilon)=\sum_{n \geq 0} y_{n}(t, z) \epsilon^{n}$ with bounded holomorphic coefficients $y_{n}(t, z)$ on $\mathscr{T} \times H_{\beta}$. This asymptotic expansion turns out to be (at most) of Gevrey order $1 / \kappa$ with $\kappa=k k^{\prime} /\left(k+k^{\prime}\right)$, meaning that we can single out two constants $C_{p}, M_{p}>0$ such that

$$
\sup _{t \in \mathscr{T}, z \in H_{\beta}}\left|y_{p}(t, z, \epsilon)-\sum_{m=0}^{n-1} y_{m}(t, z) \epsilon^{m}\right| \leq C_{p} M_{p}^{n} \Gamma\left(1+\frac{n}{\kappa}\right)|\epsilon|^{n},
$$

for all $n \geq 1$, all $\epsilon \in \mathscr{E}_{p}$.

We plan to obtain a similar statement for the problem under study (1). Namely, we will construct a set of genuine sectorial solutions to (1) and describe their asymptotic expansions as $\epsilon$ borders the origin. We first notice that our main problem (1) can be seen as a $q$-analog of (2), where the irregular differential operator $t^{k+1} \partial_{t}$ is replaced by the discrete operator $t^{k} \sigma_{q: t}$. This terminology originates from the basic observation that the expression $f(q t)-f(t) /(q t-t)$ approaches the derivative $f^{\prime}(t)$ as $q$ tends to 1 . Here, as mentioned in the title, we qualify the $q$-analogy as partial since the Fuchsian operator $t \partial_{t}$ is not discretized in the process. This suggests that, in the building procedure of the solutions (that will follow the same guideline as in [3]), the classical Laplace transform of order $k$ shall be supplanted by a $q$-Laplace transform of order $k$ as it was the case in the previous work [5] of the author where a similar problem was handled. However, due to presence of the Fuchsian operator $t \partial_{t}$, we will see that a single $q$-Laplace transform is not enough to construct true solutions and that a new mechanism of iterated $q$-Laplace and classical Laplace transforms is required. Furthermore, we witness that this enhanced multisummability procedure has a forthright effect on their asymptotic expression w.r.t $\epsilon$. Namely, the expansions in the perturbation parameter are neither of classical Gevrey order as displayed in (3) nor of $q$-Gevrey order $1 / k$ as in [5] (meaning that $\Gamma(1+(n / \kappa))$ has to be replaced by $q^{n^{2} / 2 k}$ in the control term of (3)). The asymptotic expansions we exhibit present a double scale structure which has a $q$-Gevrey leading part with order $1 / k$ and a subdominant tail of Gevrey order $1 / k \delta_{D}$, that we call Gevrey asymptotic expansion of mixed order $\left(1 / k \delta_{D} ;(q, 1 / k)\right)$ (cf. Definition 8$)$. Such a coupled asymptotic structure has already been observed in another setting by Lastra et al. in [6]. Indeed, we considered linear $q$-difference differential Cauchy problems with the shape

$$
\left(t \sigma_{q ; t}\right)^{r_{2}}\left(z \partial_{z}\right)^{r_{1}} \partial_{z}^{S} X(t, z)=B\left(z, t \sigma_{q ; t}, \sigma_{q^{-1} ; z}, \partial_{z}\right) X(t, z),
$$

for suitably chosen analytic Cauchy data

$$
\left(\partial_{z}^{j} X\right)(t, 0)=\varphi_{j}(t), \quad 0 \leq j \leq S-1,
$$

and properly selected complex number $q \in \mathbb{C}^{*}$ with $|q|>1$, where $r_{1} \geq 0$ and $r_{2}, S \geq 1$ are integers and $B$ stands for a polynomial. When $r_{1} \geq 1$, the Fuchsian operator $\left(z \partial_{z}\right)^{r_{1}}$ is responsible of the classical Gevrey part of the asymptotic expansion $\widehat{X}(t, z)=\sum_{n \geq 0} X_{n}(z) t^{n}$ of the true solution $X(t, z)$ which is shown to be of mixed order $\left(r_{1} / r_{2} ;(q, 1)\right)$ (in the sense of Definition 8 ) outside some $q$-spiral $\lambda q^{\mathbb{Z}}$ for some $\lambda \in \mathbb{C}^{*}$ w.r.t $t$ near 0 , uniformly in $z$ in the vicinity of the origin. Here, the solutions are expressed through a single $q$-Laplace transform and the $\Gamma\left(\left(r_{1} / r_{2}\right) n\right)$ contribution in the asymptotics emerges from a discrete set of singularities that accumulates at 0 in the Borel plane.

It is worthwhile mentioning that the approach which consists in building solutions by means of iterated $q$-Laplace and Laplace transforms stems from a new work by Yamazawa. In [7], he examines linear $q$-difference differential equations of the form

$$
L\left(t, \sigma_{q ; t}, \partial_{x}\right) u(t, x)=f(t, x),
$$

for the given holomorphic forcing term $f(t, x)$ near the origin and where $L\left(t, V_{1}, V_{2}\right)$ is a polynomial in $V_{1}, V_{2}$ with holomorphic coefficients w.r.t $t$ near 0 . Under special conditions on the structure of (4), he is able to construct a genuine solution $u(t, x)$ obtained as a small perturbation of iterated truncated $q$-Laplace and Laplace of order 1 transforms of the iterated Borel and $q$-Borel transforms of a formal solution $\widehat{u}(t, x)=\sum_{k \geq 1} u_{k}(x) t^{k}$ of (4). Furthermore, he gets in particular that $u(t, x)$ has $\widehat{u}(t, x)$ as asymptotic expansion of mixed order $(1 ;(q, 1))$ w.r.t $t$, uniformly in $x$ near 0 .

Notice that in our paper, the solutions are built up as complete iterated $q$-Laplace and classical Laplace transforms that are shown to be exact solutions of our problem (1). This is why the process we follow can actually be understood as an enhanced version of the multisummation mechanism introduced by Balser, see [4].

In a larger framework, this work is a contribution to the promising and fruitful realm of research in $q$-difference and $q$-difference-differential equations in the complex domain. For recent important advances in this area, we mention in particular the works by Tahara and Yamazawa [8-10]. Notice that the fields of applications of $q$-difference equations have also encountered a rapid growth in the last years. 
Some forefront studies in this respect are given, for instance, by [11-13] and references therein.

Now, we describe a little more precisely our main results obtained in Theorems 1 and 3. Namely, under convenient restrictions on the shape of (1) detailed in the statement of Theorem 1, we can manufacture a family of bounded holomorphic solutions $u_{p}(t, z, \epsilon)$ on domains $\mathscr{T} \times H_{\beta} \times \mathscr{E}_{p}$ for a suitable bounded sector $\mathscr{T}$ at $0, H_{\beta}$ a strip of width $\beta>0$, and $\mathscr{E}_{p}$ belonging to a good covering in $\mathbb{C}^{*}$, which can be displayed as a $q$-Laplace transform of order $k$ along a halfline $L_{\gamma_{p}}=\mathbb{R}_{+} \exp \left(\sqrt{-1} \gamma_{p}\right)$ and Fourier integral:

$$
\begin{aligned}
u_{p}(t, z, \epsilon)= & \frac{1}{\pi_{q^{1 / k}}(2 \pi)^{1 / 2}} \int_{-\infty}^{+\infty} \int_{L_{\gamma_{p}}} W^{d p}(u, m, \epsilon) \frac{1}{\Theta_{q^{1 / k}}(u / \epsilon t)} \\
& \cdot e^{i z m} \frac{\mathrm{d} u}{u} \mathrm{~d} m .
\end{aligned}
$$

The $q$-Borel/Fourier map $W^{d} p(u, m, \epsilon)$ is itself shaped as a classical Laplace transform of order $k \delta_{D}$ along $L_{\gamma_{p}}$ :

$$
W^{d_{p}}(u, m, \epsilon)=k \delta_{D} \int_{L_{\gamma_{p}}} w^{d_{p}}(h, m, \epsilon) \exp \left(-\left(\frac{h}{u}\right)^{k \delta_{D}}\right) \frac{\mathrm{d} h}{h},
$$

where $w^{d p}(h, m, \epsilon)$ has (at most) q-exponential growth of some order $0<k_{1}<k$ along $L_{\gamma_{p}}($ see $(180))$ and exponential decay in phase $m \in \mathbb{R}$. In Theorem 3 , we explain the reason for which all the partial functions $\epsilon \longmapsto u_{p}(t, z, \epsilon)$ share a common asymptotic expansion $\widehat{u}(t, z, \epsilon)=\sum_{m \geq 0} h_{m}(t, z) \epsilon^{m}$ on $\mathscr{E}_{p}$ with bounded holomorphic coefficients $h_{m}(t, z)$ on $\mathscr{T} \times H_{\beta}$, which turns out to be of mixed order $\left(1 / k \delta_{D}\right.$; $(q, 1 / k))$. This last result leans on a new version of the classical Ramis-Sibuya theorem fitting the above asymptotics, which is fully expounded in Theorem 2 .

Our paper is arranged as follows.

In Section 2, we recall the definition of the classical Laplace transform and its $q$-analog. We also put forward some classical identities for the Fourier transform acting on functions spaces with exponential decay.

In Section 3, we set forth our main problem (33) and we discuss the formal steps leading to its resolution. Namely, a first part is devoted to the inquiry of solutions among $q$ Laplace transforms of order $k$ and Fourier inverse integrals of Borel maps $W$ with $q$-exponential growth on unbounded sectors and exponential decay in phase leading to the first main integrodifferential $q$-difference equation (69) that $W$ is asked to fulfill. A second undertaking suggests to seek for $W$, as a classical Laplace transform of suitable order $k \delta_{D}$ of a second Borel map $w$ with again appropriate behaviour. The expression $w$ is then contrived to solve a second principal integro $q$-difference equation (81).

In Section 4 , bounds for linear convolution and $q$-difference operators acting on Banach spaces of functions with $q$-exponential growth are displayed. The second key equation (81) is then solved within these spaces at the hand of a fixed point argument.
In Section 5, genuine holomorphic solutions $W$ of the first principal auxiliary equation (69) are built up and sharp estimates for their growth are provided (cf. (146) and (147)).

In Section 6, we achieve our goal in finding a set of true holomorphic solutions (176) to our initial problem (33).

In Section 7, the existence of a common asymptotic expansion of the Gevrey type with mixed order $\left(1 / k \delta_{D} ;(q, 1 / k)\right)$ is established for the solutions set up in Section 6. The decisive technical tool for its construction is detailed in Theorem 2.

\section{Laplace Transforms of Order $k^{\prime}, \boldsymbol{q}$-Laplace Transforms of Order $k$, and Fourier Inverse Maps}

Let $k^{\prime} \geq 1$ be an integer. We remind the reader the definition of the Laplace transform of order $k^{\prime}$ as introduced in [14].

Definition 1. We set $S_{d, \delta}=\left\{\tau \in \mathbb{C}^{*}:|d-\arg (\tau)|<\delta\right\}$ as some unbounded sector with bisecting direction $d \in \mathbb{R}$ and aperture $2 \delta>0$ and $D(0, \rho)$ as a disc centered at 0 with radius $\rho>0$. Consider a holomorphic function $w: S_{d, \delta} \cup$ $D(0, \rho) \longrightarrow \mathbb{C}$ that vanishes at 0 and withstands the bounds. There exist $C>0$ and $K>0$ such that

$$
|w(\tau)| \leq C|\tau| \exp \left(K|\tau|^{k^{\prime}}\right)
$$

for all $\tau \in S_{d, \delta}$. We define the Laplace transform of $w$ of order $k^{\prime}$ in the direction $d$ as the integral transform

$$
\mathscr{L}_{k^{\prime}}^{d}(w)(T)=k^{\prime} \int_{L_{\gamma}} w(u) \exp \left(-\left(\frac{u}{T}\right)^{k^{\prime}}\right) \frac{\mathrm{d} u}{u},
$$

along a halfline $L_{\gamma}=\mathbb{R}_{+} e^{\sqrt{-1} \gamma} \subset S_{d, \delta} \cup\{0\}$, where $\gamma$ depends on Tand is chosen in such a way that $\cos \left(k^{\prime}(\gamma-\arg (T))\right) \geq \delta_{1}$, for some fixed real number $\delta_{1}>0$. The function $\mathscr{L}_{k^{\prime}}^{d}(w)(T)$ is well defined, holomorphic, and bounded on any sector:

$$
S_{d, \theta, R^{1 / k^{\prime}}}=\left\{T \in \mathbb{C}^{*}:|T|<R^{1 / k^{\prime}},|d-\arg (T)|<\frac{\theta}{2}\right\},
$$

where $0<\theta<\left(\pi / k^{\prime}\right)+2 \delta$ and $0<R<\delta_{1} / K$.

If one sets $w(\tau)=\sum_{n \geq 1} w_{n} \tau^{n}$, the Taylor expansion of $w$, which converges on the disc $D(0, \rho / 2)$, the Laplace transform $\mathscr{L}_{k^{\prime}}^{d}(w)(T)$ has the formal series

$$
\widehat{X}(T)=\sum_{n \geq 1} w_{n} \Gamma\left(\frac{n}{k^{\prime}}\right) T^{n},
$$

as Gevrey asymptotic expansion of order $1 / k^{\prime}$. This means that for all $0<\theta_{1}<\theta$, two constants $C, M>0$ can be selected with the bounds:

$$
\left|\mathscr{L}_{k^{\prime}}^{d}(w)(T)-\sum_{p=1}^{n-1} w_{p} \Gamma\left(\frac{p}{k^{\prime}}\right) T^{p}\right| \leq C M^{n} \Gamma\left(1+\frac{n}{k^{\prime}}\right)|T|^{n},
$$

for all $n \geq 2$, all $T \in S_{d, \theta_{1}, R^{1 / k^{\prime}}}$.

In particular, if $w(\tau)$ represents an entire function w.r.t $\tau \in \mathbb{C}$ with bound (11), its Laplace transform $\mathscr{L}_{k^{\prime}}^{d}(w)(T)$ does not depend on the direction $d$ in $\mathbb{R}$ and represents a 
bounded holomorphic function on $D\left(0, R^{1 / k^{\prime}}\right)$, whose Taylor expansion is represented by the convergent series $X(T)=$ $\sum_{n \geq 1} w_{n} \Gamma\left(n / k^{\prime}\right) T^{n}$ on $D\left(0, R^{1 / k^{\prime}}\right)$.

Let $k \geq 1$ be an integer and $q>1$ be a real number. At the next stage, we display the definition of the $q$-Laplace transform of order $k$ which was used in a former work of Malek [5].

Let us first recall some essential properties of the Jacobi Theta function of order $k$ defined as the Laurent series:

$$
\Theta_{q^{1 / k}}(x)=\sum_{n \in \mathbb{Z}} q^{-(n(n-1) / 2 k)} x^{n}
$$

for all $x \in \mathbb{C}^{*}$. This analytic function can be factorized as a product known as Jacobi's triple product formula:

$$
\Theta_{q^{1 / k}}(x)=\prod_{n \geq 0}\left(1-q^{(-n-1) / k}\right)\left(1+x q^{-(n / k)}\right)\left(1+\frac{q^{(-n-1) / k}}{x}\right),
$$

for all $x \in \mathbb{C}^{*}$, from which we deduce that its zeros are the set of real numbers $\left\{-q^{m / k} / m \in \mathbb{Z}\right\}$. We recall the next lower bound estimates on a domain, bypassing the set of zeroes of $\Theta_{q^{1 / k}}(x)$, from [5] Lemma 3, which are crucial in the sequel.

Lemma 1. Let $\Delta>0$. There exists a constant $C_{q, k}>0$ depending on $q, k$ and independent of $\Delta$ such that

$$
\left|\Theta_{q^{1 / k}}(x)\right| \geq C_{q, k} \Delta \exp \left(\frac{k}{2} \frac{\log ^{2}(|x|)}{\log (q)}\right)|x|^{1 / 2},
$$

for all $x \in \mathbb{C}^{*}$ satisfying $\left|1+x q^{m / k}\right|>\Delta$, for all $m \in \mathbb{Z}$.

Definition 2. Let $\rho>0$ be a real number and $S_{d}$ be an unbounded sector centered at 0 with bisecting direction $d \in \mathbb{R}$. Let $f: D(0, \rho) \cup S_{d} \longrightarrow \mathbb{C}$ be a holomorphic function, continuous on the adherence $\overline{D(0, \rho)}$, such that there exist constants $K, \alpha>0$ and $\delta>1$ with

$$
|f(x)| \leq K|x| \exp \left(\frac{k}{2} \frac{\log ^{2}(|x|+\delta)}{\log (q)}+\alpha \log (|x|+\delta)\right),
$$

for all $x \in S_{d} \cup D(0, \rho)$. Let $\gamma \in \mathbb{R}$ with $e^{\sqrt{-1} \gamma} \in S_{d}$. We put $\pi_{q^{1 / k}}=\log (q) / k$. We define the $q$-Laplace transform of order $k$ of $f$ in direction $\gamma$ as

$$
\mathscr{L}_{q ; 1 / k}^{\gamma}(f(x))(T)=\frac{1}{\pi_{q^{1 / k}}} \int_{L_{\gamma}} \frac{f(u)}{\Theta_{q^{1 / k}}(u / T)} \frac{\mathrm{d} u}{u},
$$

where $L_{\gamma}=\mathbb{R}_{+} e^{\sqrt{-1} \gamma}$ is a halfline in the direction $\gamma$.

The following lemma is a slightly modified version of Lemma 4 from [5].

Lemma 2. Let $\Delta>0$ chosen as in Lemma 1 above. The integral transform $\mathscr{L}_{q ; 1 / k}^{\gamma}(f(x))(T)$ defines a bounded holomorphic function on the domain $\mathscr{R}_{\gamma, \Delta} \cap D\left(0, r_{1}\right)$ for any radius $0<r_{1} \leq q^{-(1 / k)(\alpha+1)} / 2$, where

$$
\mathscr{R}_{\gamma, \Delta}=\left\{T \in \frac{\mathbb{C}^{*}}{\left|1+\left(e^{\sqrt{-1}} \gamma / T\right) r\right|}>\Delta \text {, for all } r \geq 0\right\} .
$$

Notice that the value $\mathscr{L}_{q ; 1 / k}^{\gamma}(f(x))(T)$ does not depend on $\gamma \in \mathbb{R}$ such that $e^{\sqrt{-1} \gamma} \in S_{d}$ due to the Cauchy formula.

The next lemma describes conditions under which the qLaplace transform defines a convergent series near the origin.

Lemma 3. Let $f: \mathbb{C} \longrightarrow \mathbb{C}$ be an entire function with Taylor expansion $f(x)=\sum_{n \geq 1} f_{n} x^{n}$ fulfilling bound (19) for all $x \in \mathbb{C}$. Then, its $q$-Laplace transform of order $k$, $\mathscr{L}_{q ; 1 / k}^{d}(f)(T)$, does not depend on the direction $d \in \mathbb{R}$ and represents a bounded holomorphic function on $D\left(0, r_{1}\right)$ with the restriction $0<r_{1} \leq q^{-(1 / k)(\alpha+1)} / 2$ whose Taylor expansion is given by the convergent series $Y(T)=\sum_{n \geq 1} f_{n} q^{n(n-1) / 2 k} T^{n}$.

Proof. The proof is a direct consequence of the next formulas:

$$
\frac{1}{\pi_{q^{1 / k}}} \int_{L_{\gamma}} \frac{u^{n-1}}{\Theta_{q^{1 / k}}(u / T)} \mathrm{d} u=T^{n} \frac{1}{\pi_{q^{1 / k}}} \int_{L_{\gamma^{\prime}}} \frac{v^{n-1}}{\Theta_{q^{1 / k}}(v)} \mathrm{d} v=T^{n} q^{n(n-1) / 2 k},
$$

whenever $T \in \mathscr{R}_{\gamma, \delta}$ and $\gamma^{\prime}=\gamma-\arg (T)$, where the last equality follows (for instance) from identity (4.7) from [15], for all $n \geq 1$.

We restate the definition of some family of Banach spaces mentioned in [14].

Definition 3. Let $\beta, \mu \in \mathbb{R}$. We set $E_{(\beta, \mu)}$ as the vector space of continuous functions $h: \mathbb{R} \longrightarrow \mathbb{C}$ such that

$$
\|h(m)\|_{(\beta, \mu)}=\sup _{m \in \mathbb{R}}(1+|m|)^{\mu} \exp (\beta|m|)|h(m)|,
$$

is finite. The space $E_{(\beta, \mu)}$ endowed with the norm $\|\cdot\|_{(\beta, \mu)}$ becomes a Banach space.

Finally, we remind the reader the definition of the inverse Fourier transform acting on the latter Banach spaces and some of its handy formulas relative to derivation and convolution product as stated in [14].

Definition 4. Let $f \in E_{(\beta, \mu)}$ with $\beta>0$ and $\mu>1$. The inverse Fourier transform $f$ is given by

$$
\mathscr{F}^{-1}(f)(x)=\frac{1}{(2 \pi)^{1 / 2}} \int_{-\infty}^{+\infty} f(m) \exp (\text { ixm }) \mathrm{d} m,
$$

for all $x \in \mathbb{R}$. The function $\mathscr{F}^{-1}(f)$ extends to an analytic bounded function on the strips

$$
H_{\beta^{\prime}}=\left\{z \in \frac{\mathbb{C}}{|\operatorname{Im}(z)|}<\beta^{\prime}\right\},
$$

for all given $0<\beta^{\prime}<\beta$.

(a) Define the function $m \longmapsto \phi(m)=\operatorname{imf}(m)$ which belongs to the space $E_{(\beta, \mu-1)}$. Then, the next identity

$$
\partial_{z} \mathscr{F}^{-1}(f)(z)=\mathscr{F}^{-1}(\phi)(z),
$$

occurs.

(b) Take $g \in E_{(\beta, \mu)}$ and set

$$
\psi(m)=\frac{1}{(2 \pi)^{1 / 2}} \int_{-\infty}^{+\infty} f\left(m-m_{1}\right) g\left(m_{1}\right) \mathrm{d} m_{1},
$$


as the convolution product of $f$ and $g$. Then, $\psi$ belongs to $E_{(\beta, \mu)}$, and moreover,

$$
\mathscr{F}^{-1}(f)(z) \mathscr{F}^{-1}(g)(z)=\mathscr{F}^{-1}(\psi)(z),
$$

for all $z \in H_{\beta}$.

\section{Layout of the Principal Initial Value Problem and Associated Auxiliary Problems}

We set $k \geq 1$ as an integer. Let $m_{D}, \delta_{D} \geq 1$ be integers. We set

$$
k^{\prime}=k \delta_{D} \text {. }
$$

We consider a finite set $I$ of $\mathbb{N}^{3}$ that fulfills the next feature,

$$
l_{0} \geq 1+l_{2} k^{\prime}
$$

whenever $\left(l_{0}, l_{1}, l_{2}\right) \in I$ and we set nonnegative integers $\Delta_{l} \geq 0$ with

$$
\Delta_{l}-l_{0} \geq 0
$$

for all $l=\left(l_{0}, l_{1}, l_{2}\right) \in I$.

Let $Q(X), R_{D}(X), R_{l}(X) \in \mathbb{C}[X]$, and $l \in I$ be polynomials such that

$$
\begin{aligned}
\operatorname{deg}(Q) & \geq \operatorname{deg}\left(R_{D}\right) \geq \operatorname{deg}\left(R_{l}\right), \\
Q(i m) & \neq 0, \\
R_{D}(i m) & \neq 0,
\end{aligned}
$$

for all $m \in \mathbb{R}$, all $l \in I$.

We consider a family of linear singularly perturbed initial value problems

$$
\begin{aligned}
& Q\left(\partial_{z}\right) u(t, z, \epsilon)=R_{D}\left(\partial_{z}\right) \epsilon^{\delta_{D} k m_{D}}\left(t^{\delta_{D} k} \sigma_{q ; t}^{\delta_{D}} t \partial_{t}\right)^{m_{D}} u(t, z, \epsilon) \\
& \quad+\sum_{l=\left(l_{0}, l_{1}, l_{2}\right) \in I} \epsilon^{\Delta_{l}} c_{l}(z, \epsilon) R_{l}\left(\partial_{z}\right) t^{l_{0}} \sigma_{q ; t}^{l_{1}}\left(t \partial_{t}\right)^{l_{2}} u(t, z, \epsilon)+f(t, z, \epsilon),
\end{aligned}
$$

for vanishing initial data $u(0, z, \epsilon) \equiv 0$. Here, $q>1$ stands for a real number and the operator $\sigma_{q ; t}$ is defined as the dilation by $q$ acting on the variable $t$ through $\sigma_{q ; t} u(t, z, \epsilon)=$ $u(q t, z, \epsilon)$.

The coefficients $c_{l}(z, \epsilon)$ are built in the following manner. For each $l \in I$, we consider a function $m \longmapsto C_{l}(m, \epsilon)$ that belongs to the Banach space $E_{(\beta, \mu)}$ for some $\beta, \mu>0$, which depends holomorphically on the parameter $\epsilon$ on some disc $D\left(0, \epsilon_{0}\right)$ with radius $\epsilon_{0}>0$ and for which one can find a constant $C_{l}>0$ with

$$
\sup _{\epsilon \in D\left(0, \epsilon_{0}\right)}\left\|C_{l}(m, \epsilon)\right\|_{(\beta, \mu)} \leq C_{l} .
$$

We construct

$$
c_{l}(z, \epsilon)=\frac{1}{(2 \pi)^{1 / 2}} \int_{-\infty}^{+\infty} C_{l}(m, \epsilon) e^{i z m} \mathrm{~d} m,
$$

as the inverse Fourier transform of the map $C_{l}(m, \epsilon)$ for all $l \in I$. As a result, $c_{l}(z, \epsilon)$ is bounded holomorphically w.r.t $\epsilon$ on $D\left(0, \epsilon_{0}\right)$ and w.r.t $z$ on any strip $H_{\beta^{\prime}}$ for $0<\beta^{\prime}<\beta$ in view of Definition 4.
The presentation of the forcing term requires some preliminary groundwork. We consider a sequence of functions $m \longmapsto \psi_{n}(m, \epsilon)$, for $n \geq 1$, that belongs to the Banach space $E_{(\beta, \mu)}$ with the parameters $\beta, \mu>0$ given above and which relies analytically and is bounded w.r.t $\epsilon$ on the disc $D\left(0, \epsilon_{0}\right)$. We assume that the next bounds,

$$
\sup _{\epsilon \in D\left(0, \epsilon_{0}\right)}\left\|\psi_{n}(m, \epsilon)\right\|_{(\beta, \mu)} \leq K_{0}\left(\frac{1}{T_{0}}\right)^{n},
$$

hold for all $n \geq 1$ and given constants $K_{0}, T_{0}>0$. We define the formal series

$$
\psi(\tau, m, \epsilon)=\sum_{n \geq 1} \psi_{n}(m, \epsilon) \frac{\tau^{n}}{\left(q^{1 / k_{1}}\right)^{n(n-1) / 2}},
$$

for some real number $0<k_{1}<k$. We introduce the next Banach space.

Definition 5. Let $k_{1}, \beta, \mu, r, \alpha>0$ and $q, \delta>1$ be real numbers. Let $U_{d}$ be an open unbounded sector with bisecting direction $d \in \mathbb{R}$ centered at 0 in $\mathbb{C}$. We denote $\operatorname{Exp}_{\left(k_{1}, \beta, \mu, \alpha, r\right)}^{q}$ the vector space of complex valued continuous functions $(u, m) \longmapsto h(u, m)$ on the adherence $\overline{U_{d}} \cup \overline{D(0, r)} \times \mathbb{R}$, which are holomorphic w.r.t u on $U_{d} \cup D(0, r)$ and such that the norm

$$
\begin{aligned}
\|h(u, m)\|_{\left(k_{1}, \beta, \mu, \alpha, r\right)}= & \sup _{u \in \bar{U}_{d}} \frac{\sin _{(0, r), m \in \mathbb{R}}}{}(1+|m|)^{\mu} e^{\beta|m|} \frac{1}{|u|} \\
& \times \exp \left(-\frac{k_{1}}{2} \frac{\log ^{2}(|u|+\delta)}{\log (q)}-\alpha \log (|u|+\delta)\right) \\
& \cdot|h(u, m)|,
\end{aligned}
$$

is finite. One can check that the normed space $\left(\operatorname{Exp}_{\left(k_{1}, \beta, \mu, \alpha, r\right)}^{q},\|\cdot\|_{\left(k_{1}, \beta, \mu, \alpha, r\right)}\right)$ represents a Banach space.

Remark 1. The spaces above are faint modifications of the Banach spaces already introduced in the works of Dreyfus and Lastra [16-18].

The next lemma is a proper adjustment of Lemma 5 out of [5] to the new Banach spaces from Definition 5.

Lemma 4. Let $T_{0}$ be fixed as in (36). We take a number $\alpha>0$ such that

$$
T_{0}>\frac{q^{1 / 2 k_{1}}}{q^{(\alpha+1) / k_{1}}} .
$$

Let $k_{1}, \beta, \mu$ be chosen as above. Then, the function $(u, m) \longmapsto \psi(u, m, \epsilon)$ belongs to the Banach space $\operatorname{Exp}_{\left(k_{1}, \beta, \mu, \alpha, r\right)}^{q}$ for any unbounded sector $U_{d}$, any disc $D(0, r)$. Moreover, one can find a constant $C_{1}>0$ (depending on $\left.q, k_{1}, \alpha, T_{0}\right)$ with

$$
\sup _{\epsilon \in D\left(0, \epsilon_{0}\right)}\|\psi(u, m, \epsilon)\|_{\left(k_{1}, \beta, \mu, \alpha, r\right)} \leq K_{0} C_{1} .
$$

Proof. ound (36) implies that 


$$
\begin{aligned}
\|\psi(u, m, \epsilon)\|_{\left(k_{1}, \beta, \mu, \alpha, r\right)} & \leq \sum_{n \geq 1}\left\|\psi_{n}(m, \epsilon) \frac{u}{\left(q^{1 / k_{1}}\right)^{n(n-1) / 2}}\right\|_{\left(k_{1}, \beta, \mu, \alpha, r\right)} \\
& \leq \sum_{n \geq 1} K_{0}\left(\frac{1}{T_{0}}\right)^{n} \frac{1}{\left(q^{1 / k_{1}}\right)^{n(n-1) / 2}} \sup _{u \in \overline{U_{d}} \cup \frac{1}{D(0, r)}}|u|^{n-1} \exp \left(-\frac{k_{1}}{2} \frac{\log ^{2}(|u|+\delta)}{\log (q)}-\alpha \log (|u|+\delta)\right) .
\end{aligned}
$$

According to the elementary fact that the polynomial $h(x)=x(n-1-\alpha)-\left(k_{1} / 2\right)\left(x^{2} / \log (q)\right)$ admits its maximum value $\left(\log (q) / 2 k_{1}\right)(n-1-\alpha)^{2}$ at $x=\left(\log (q) / k_{1}\right)$ $(n-1-\alpha)$, we deduce by means of the change of variable $x=\log (|u|+\delta)$ that

$$
\begin{gathered}
\sup _{u \in \overline{U_{d}} \cup \overline{D(0, r)}}|u|^{n-1} \exp \left(-\frac{k_{1}}{2} \frac{\log ^{2}(|u|+\delta)}{\log (q)}-\alpha \log (|u|+\delta)\right) \\
\leq \sup _{x \in \mathbb{R}} \exp \left(x(n-1-\alpha)-\frac{k_{1} x^{2}}{2 \log (q)}\right) \leq q^{(n-1-\alpha)^{2} / 2 k_{1}},
\end{gathered}
$$

for all $n \geq 1$. Therefore, we deduce that

$$
\|\psi(u, m, \epsilon)\|_{\left(k_{1}, \beta, \mu, \alpha, r\right)} \leq K_{0} q^{(1+\alpha)^{2} / 2 k_{1}} \sum_{n \geq 1}\left(\frac{q^{1 / 2 k_{1}}}{T_{0} q^{(\alpha+1) / k_{1}}}\right)^{n},
$$

which converges, provided that (39) holds, whenever $\epsilon \in D\left(0, \epsilon_{0}\right)$.

We define

$$
\Psi_{d}(\tau, m, \epsilon)=k^{\prime} \int_{L_{d}} \psi(u, m, \epsilon) \exp \left(-\left(\frac{u}{\tau}\right)^{k^{\prime}}\right) \frac{\mathrm{d} u}{u},
$$

as the Laplace transform of $\psi(u, m, \epsilon)$ w.r.t $u$ of order $k^{\prime}$ in direction $d \in \mathbb{R}$. Notice that two constants $K_{1}, K_{2}>0$ (depending on $k_{1}, q, \alpha, \delta, k^{\prime}$ ) can be found such that

$$
\frac{k_{1}}{2} \frac{\log ^{2}(|u|+\delta)}{\log (q)}+\alpha \log (|u|+\delta) \leq K_{1}|u|^{k^{\prime}}+K_{2},
$$

for all $u \in \mathbb{C}$. As a result, owing to bound (40) and the last part of Definition 1, we deduce that $\Psi_{d}$ does not depend on the direction $d$ and can be written as a convergent series:

$$
\Psi_{d}(\tau, m, \epsilon)=\sum_{n \geq 1} \psi_{n}(m, \epsilon) \frac{\Gamma\left(n / k^{\prime}\right)}{\left(q^{1 / k_{1}}\right)^{n(n-1) / 2}} \tau^{n},
$$

w.r.t $\tau$ near the origin. Now, we fix some real number $k_{2}$ such that $0<k_{1}<k_{2}<k$. Then, one can sort a constant $C_{2}$ (depending on $q, k_{1}, k_{2}, k^{\prime}$ ) such that

$$
\frac{\Gamma\left(n / k^{\prime}\right)}{\left(q^{1 / k_{1}}\right)^{n(n-1) / 2}} \leq C_{2} \frac{1}{\left(q^{1 / k_{2}}\right)^{n(n-1) / 2}},
$$

for all $n \geq 1$. This inequality is a consequence of the Stirling formula, which states that

$$
\Gamma(x) \sim \sqrt{2 \pi} e^{-x} x^{-1 / 2} x^{x},
$$

as $x$ tends to $+\infty$ and from the existence of a constant $K_{3}>0$ (depending on $q, k_{1}, k_{2}, k^{\prime}$ ) with

$$
-\frac{n}{k^{\prime}}+\frac{n}{k^{\prime}} \log \left(\frac{n}{k^{\prime}}\right)+\frac{n(n-1) \log (q)}{2 k_{2}} \leq \frac{n(n-1) \log (q)}{2 k_{1}}+K_{3} \text {, }
$$

for all $n \geq 1$. Consequently, it turns out that $\Psi_{d}(\tau, m, \epsilon)$ represents an entire function w.r.t $\tau$ such that

$$
\left|\Psi_{d}(\tau, m, \epsilon)\right| \leq K_{0} C_{2}(1+|m|)^{-\mu} e^{-\beta|m|} \sum_{n \geq 1} \frac{\left(|\tau| / T_{0}\right)^{n}}{\left(q^{1 / k_{2}}\right)^{n(n-1) / 2}},
$$

for all $\tau \in \mathbb{C}$. Furthermore, owing to bound (42), we know that

$$
|\tau|^{n} \leq q^{(n-\alpha)^{2} / 2 k_{2}} \exp \left(\frac{k_{2}}{2} \log ^{2}(|\tau|+\delta)+\alpha \log (|\tau|+\delta)\right),
$$

for all $n \geq 1$, all $\tau \in \mathbb{C}$. Henceforth, we get the next global bounds

$$
\begin{aligned}
\left|\Psi_{d}(\tau, m, \epsilon)\right| \leq & K_{0} C_{2} q^{\alpha^{2} / 2 k_{2}} \sum_{n \geq 1}\left(\frac{q^{(1-2 \alpha) / 2 k_{2}}}{T_{0}}\right)^{n}(1+|m|)^{-\mu} e^{-\beta|m|} \\
& \times \exp \left(\frac{k_{2}}{2} \log ^{2}(|\tau|+\delta)+\alpha \log (|\tau|+\delta)\right),
\end{aligned}
$$

provided that

$$
T_{0}>q^{(1-2 \alpha) / 2 k_{2}}
$$

for all $\tau \in \mathbb{C}, m \in \mathbb{R}$, and $\epsilon \in D\left(0, \epsilon_{0}\right)$.

Next, we set

$$
\begin{aligned}
F_{d}(T, z, \epsilon)= & \frac{1}{\pi_{q^{1 / k}}(2 \pi)^{1 / 2}} \int_{-\infty}^{+\infty} \int_{L_{d}} \Psi_{d}(u, m, \epsilon) \\
& \cdot \frac{1}{\Theta_{q^{1 / k}}(u / T)} e^{i z m} \frac{\mathrm{d} u}{u} \mathrm{~d} m,
\end{aligned}
$$

as the $q$-Laplace transform of $\Psi_{d}(u, m, \epsilon)$ w.r.t $u$ of order $k$ in direction $d$ and Fourier inverse integral w.r.t $m$. We put

$$
F_{n}(z, \epsilon)=\frac{1}{(2 \pi)^{1 / 2}} \int_{-\infty}^{+\infty} \psi_{n}(m, \epsilon) e^{i z m} \mathrm{~d} m
$$

for all $n \geq 1$. We first provide bounds for this sequence of functions. Namely, we can get a constant $C_{\mu, \beta, \beta^{\prime}}>0$ (relying on $\mu, \beta, \beta^{\prime}$ ) with 


$$
\begin{aligned}
\left|F_{n}(z, \epsilon)\right| & \leq \frac{K_{0}}{(2 \pi)^{1 / 2}}\left(\frac{1}{T_{0}}\right)^{n} \int_{-\infty}^{+\infty}(1+|m|)^{-\mu} e^{-\beta|m|} e^{-\operatorname{Im}(z) m} \mathrm{~d} m \\
& \leq \frac{K_{0}}{(2 \pi)^{1 / 2}}\left(\frac{1}{T_{0}}\right)^{n} \int_{-\infty}^{+\infty}(1+|m|)^{-\mu} e^{\left(\beta^{\prime}-\beta\right)|m|} \mathrm{d} m \\
& \leq \frac{C_{\mu, \beta, \beta^{\prime}} K_{0}}{(2 \pi)^{1 / 2}}\left(\frac{1}{T_{0}}\right)^{n}
\end{aligned}
$$

for all $n \geq 1$, whenever $\epsilon \in D\left(0, \epsilon_{0}\right)$ and $z$ belongs to the horizontal strip $H_{\beta^{\prime}}$ for some $0<\beta^{\prime}<\beta$ (see Definition 4). Owing to Lemma 3 , we deduce that the function $F_{d}(T, z, \epsilon)$ converges near the origin w.r.t $T$, where it carries the next Taylor expansion:

$$
F_{d}(T, z, \epsilon)=\sum_{n \geq 1} F_{n}(z, \epsilon) \Gamma\left(\frac{n}{k^{\prime}}\right)\left(q^{(1 / k)-\left(1 / k_{1}\right)}\right)^{n(n-1) / 2} T^{n},
$$

for all $\epsilon \in D\left(0, \epsilon_{0}\right)$ and $z \in H_{\beta^{\prime}}$. In particular, the function $F_{d}$ is independent of the direction $d$ chosen.

We now show that $F_{d}(T, z, \epsilon)$ represents an entire function w.r.t $T$ and supply explicit upper bounds. Namely, in accordance with (47), we obtain

$$
\left|F_{d}(T, z, \epsilon)\right| \leq \sum_{n \geq 1} \frac{K_{0} C_{\mu, \beta, \beta^{\prime}} C_{2}}{(2 \pi)^{1 / 2}} \frac{1}{\left(q^{\left(1 / k_{2}\right)-(1 / k)}\right)^{n(n-1) / 2}}\left(\frac{|T|}{T_{0}}\right)^{n} .
$$

Again, estimate (42) yields

$$
|T|^{n} \leq q^{\left(1 / 2 \kappa_{2}\right)(n-\alpha)^{2}} \exp \left(\frac{\kappa_{2}}{2} \frac{\log ^{2}(|T|+\delta)}{\log (q)}+\alpha \log (|T|+\delta)\right),
$$

for all $T \in \mathbb{C}$, all $n \geq 1$, where $\kappa_{2}>0$ is defined by $1 / \kappa_{2}=\left(1 / k_{2}\right)-(1 / k)$. By gathering the two last above inequalities, the next global estimates can be figured out:

$$
\begin{aligned}
\left|F_{d}(T, z, \epsilon)\right| \leq & \frac{K_{0} C_{\mu, \beta, \beta^{\prime}} C_{2}}{(2 \pi)^{1 / 2}} q^{\alpha^{2} / 2 \kappa_{2}} \sum_{n \geq 1}\left(\frac{q^{(1-2 \alpha) / 2 \kappa_{2}}}{T_{0}}\right)^{n} \\
& \cdot \exp \left(\frac{\kappa_{2}}{2} \frac{\log ^{2}(|T|+\delta)}{\log (q)}+\alpha \log (|T|+\delta)\right),
\end{aligned}
$$

for all $T \in \mathbb{C}$, all $z \in H_{\beta^{\prime}}$ and $\epsilon \in D\left(0, \epsilon_{0}\right)$, provided that

$$
T_{0}>q^{(1-2 \alpha) / 2 \kappa_{2}} .
$$

Lastly, we define the forcing term $f$ as a time rescaled version of $F_{d}$,

$$
f(t, z, \epsilon)=F_{d}(\epsilon t, z, \epsilon),
$$

that represents a bounded holomorphic function w.r.t $z \in H_{\beta^{\prime}}$ and $\epsilon \in D\left(0, \epsilon_{0}\right)$ and an entire function w.r.t $t$ with $q$-exponential growth of order $\kappa_{2}$.
Throughout this paper, we are looking for time rescaled solutions of (33) of the form

$$
u(t, z, \epsilon)=U(\epsilon t, z, \epsilon) .
$$

As a consequence, the expression $U(T, z, \epsilon)$, through the change of variable $T=\epsilon t$, is asked to solve the next singular problem:

$$
\begin{aligned}
Q\left(\partial_{z}\right) U(T, z, \epsilon)= & R_{D}\left(\partial_{z}\right)\left(T^{\delta_{D} k} \sigma_{q ; T}^{\delta_{D}} T \partial_{T}\right)^{m_{D}} U(T, z, \epsilon) \\
& +\sum_{l=\left(l_{0}, l_{1}, l_{2}\right) \in I} \epsilon^{\Delta_{l}-l_{0}} c_{l}(z, \epsilon) R_{l}\left(\partial_{z}\right) \\
& \cdot T^{l_{0}} \sigma_{q ; T}^{l_{1}}\left(T \partial_{T}\right)^{l_{2}} U(T, z, \epsilon)+F_{d}(T, z, \epsilon) .
\end{aligned}
$$

At the onset, we seek for a solution $U(T, z, \epsilon)$ that can be expressed as an integral representation via a $q$-Laplace transform of order $k$ and Fourier inverse integral:

$U_{\gamma}(T, z, \epsilon)=\frac{1}{\pi_{q^{1 / k}}(2 \pi)^{1 / 2}} \int_{-\infty}^{+\infty} \int_{L_{\gamma}} W(u, m, \epsilon) \frac{1}{\Theta_{q^{1 / k}}(u / T)} e^{i z m} \frac{\mathrm{d} u}{u} \mathrm{~d} m$,

where the inner integration is performed along a halfline $L_{\gamma}=$ $\mathbb{R}_{+} e^{\sqrt{-1} \gamma}$ in direction $\gamma \in \mathbb{R}$. Overall this section, we assume that the partial functions $u \longmapsto W(u, m, \epsilon)$ have at most $q$ exponential growth of order $k$ on some unbounded sector $S_{d}$ centered 0 with bisecting direction $d$ and $m \longmapsto W(u, m, \epsilon)$ belong to the Banach space $E_{(\beta, \mu)}$ mentioned in Definition 3, whenever $\epsilon \in D\left(0, \epsilon_{0}\right)$. Precise bounds will be given later in Section 5. Here, we assume that $L_{\gamma} \subset S_{d} \cup\{0\}$.

Our aim is now the presentation of a related problem fulfilled by the expression $W(u, m, \epsilon)$. We first need to state two identities which concern the action of $q$-difference and Fuchsian operators on $q$-Laplace tranforms.

Lemma 5. The actions of the q-difference operators $T^{l_{0}} \sigma_{q ; T}^{l_{1}}$ for integers $l_{0}, l_{1} \geq 0$ and the Fuchsian differential operator $T \partial_{T}$ are given by

$$
\begin{aligned}
T^{l_{0}} \sigma_{q ; T}^{l_{1}} U_{\gamma}(T, z, \epsilon)= & \frac{1}{\pi_{q^{1 / k}}(2 \pi)^{1 / 2}} \int_{-\infty}^{+\infty} \int_{L_{\gamma}} \frac{u^{l_{0}}}{\left(q^{1 / k}\right)^{l_{0}\left(l_{0}-1\right) / 2}} \\
& \times \sigma_{q ; u}^{l_{1}-\left(l_{0} / k\right)} W(u, m, \epsilon) \frac{1}{\Theta_{q^{1 / k}}(u / T)} e^{i z m} \frac{\mathrm{d} u}{u} \mathrm{~d} m,
\end{aligned}
$$

$$
\begin{aligned}
T \partial_{T} U_{\gamma}(T, z, \epsilon)= & \frac{1}{\pi_{q^{1 / k}}(2 \pi)^{1 / 2}} \int_{-\infty}^{+\infty} \int_{L_{\gamma}} u \partial_{u} W(u, m, \epsilon) \\
& \cdot \frac{1}{\Theta_{q^{1 / k}}(u / T)} e^{i z m} \frac{\mathrm{d} u}{u} \mathrm{~d} m .
\end{aligned}
$$

Proof. The first identity is a direct consequence of the commutation formula (76) displayed in Proposition 6 from [5]. For the second, a derivation under the integral followed by an integration by parts implies the sequence of equalities: 


$$
\begin{aligned}
T \partial_{T} U_{\gamma}(T, z, \epsilon)= & \frac{1}{\pi_{q^{1 / k}}(2 \pi)^{1 / 2}} \int_{-\infty}^{+\infty} \int_{L_{\gamma}}-\frac{1}{T} W(u, m, \epsilon)\left(\frac{1}{\Theta_{q^{1 / k}}}\right)^{\prime}(u / T) e^{i z m} \mathrm{~d} u \mathrm{~d} m \\
= & \frac{-1}{\pi_{q^{1 / k}}(2 \pi)^{1 / 2}} \int_{-\infty}^{+\infty} \int_{L_{\gamma}} W(u, m, \epsilon) \partial_{u}\left(\left(\frac{1}{\Theta_{q^{1 / k}}}\right)(u / T)\right) e^{i z m} \mathrm{~d} u \mathrm{~d} m \\
= & \frac{-1}{\pi_{q^{1 / k}}(2 \pi)^{1 / 2}} \int_{-\infty}^{+\infty}\left[W(u, m, \epsilon)\left(\frac{1}{\Theta_{q^{1 / k}}}\right)(u / T)\right]_{u=0}^{u=\infty} e^{i z m} \mathrm{~d} m \\
& +\frac{1}{\pi_{q^{1 / k}}(2 \pi)^{1 / 2}} \int_{-\infty}^{+\infty} \int_{L_{\gamma}} \partial_{u} W(u, m, \epsilon)\left(\frac{1}{\Theta_{q^{1 / k}}}\right)(u / T) e^{i z m} \mathrm{~d} u \mathrm{~d} m,
\end{aligned}
$$

from which the forecast formula follows since the map $u \longmapsto W(u, m, \epsilon)$ is assumed to possess a growth of $q$-exponential order $k$ and vanishes at $u=0$.
The application of the above identities (66) and (67) in a row with (26) and (28) leads to the first integrodifferential $q$ difference equation fulfilled by the expression $W(u, m, \epsilon)$ as long as $U_{\gamma}(T, z, \epsilon)$ solves (64):

$$
\begin{aligned}
Q(i m) W(\tau, m, \epsilon)= & R_{D}(i m) \frac{1}{\left(q^{1 / k}\right)^{m_{D} \delta_{D} k\left(\delta_{D} k-1\right) / 2}}\left(\tau^{\delta_{D} k+1} \partial_{\tau}\right)^{m_{D}} W(\tau, m, \epsilon) \\
& +\sum_{l=\left(l_{0}, l_{1}, l_{2}\right) \in I} \epsilon^{\Delta_{l}-l_{0}} \frac{1}{(2 \pi)^{1 / 2}} \int_{-\infty}^{+\infty} C_{l}\left(m-m_{1}, \epsilon\right) \\
& \times \frac{\tau^{l_{0}}}{\left(q^{1 / k}\right)^{l_{0}\left(l_{0}-1\right) / 2}} \sigma_{q ; \tau}^{l_{1}-\left(l_{0} / k\right)}\left(\left(\tau \partial_{\tau}\right)^{l_{2}} W\left(\tau, m_{1}, \epsilon\right)\right) R_{l}\left(i m_{1}\right) \mathrm{d} m_{1}+\Psi_{d}(\tau, m, \epsilon) .
\end{aligned}
$$

We turn now to the second stage of the procedure. Solutions of this latter equation are expected to be found in the class of Laplace transforms of order $k^{\prime}$ since by construction $\Psi_{d}(\tau, m, \epsilon)$ owns this structure after (44). Namely, we take for granted that

$$
W(\tau, m, \epsilon)=k^{\prime} \int_{L_{\gamma}} w(u, m, \epsilon) \exp \left(-\left(\frac{u}{\tau}\right)^{k^{\prime}}\right) \frac{\mathrm{d} u}{u},
$$

where $L_{\gamma}=\mathbb{R}_{+} e^{\sqrt{-1} \gamma}$ stands for a halfline with direction $\gamma \in \mathbb{R}$, which belongs to $U_{d} \cup\{0\}$ where $U_{d}$ represents an unbounded sector centered at 0 with bisecting direction $d$. Within this step, we assume that the expression $(u, m) \longmapsto$ $w(u, m, \epsilon)$ belongs to the Banach space $\operatorname{Exp}_{\left(k_{1}, \beta, \mu, \alpha, r\right)}^{q}$ introduced in Definition 5, for all $\epsilon \in D\left(0, \epsilon_{0}\right)$, where the constants $k_{1}, \beta, \mu$, and $\alpha$ are selected accordingly to the construction of the forcing term $f(t, z, \epsilon)$.

The next lemma has already been stated in our previous work [3].

Lemma 6. For all integers $l \geq 1$, positive integers $a_{q, l} \geq 1$ and $1 \leq q \leq l$ can be found such that

$$
\left(t \partial_{t}\right)^{l}=\sum_{q=1}^{l} a_{q, l} t^{q} \partial_{t}^{q}
$$

With the help of this last expansion, equation (69) can be recast in the form

$$
\begin{aligned}
Q(i m) W(\tau, m, \epsilon)= & R_{D}(i m) \frac{1}{\left(q^{1 / k}\right)^{m_{D} \delta_{D} k\left(\delta_{D} k-1\right) / 2}}\left(\tau^{\delta_{D} k+1} \partial_{\tau}\right)^{m_{D}} W(\tau, m, \epsilon) \\
& +\sum_{l=\left(l_{0}, l_{1}, l_{2}\right) \in I} \epsilon^{\Delta_{l}-l_{0}} \frac{1}{(2 \pi)^{1 / 2}} \int_{-\infty}^{+\infty} C_{\mathbf{l}}\left(m-m_{1}, \epsilon\right) R_{\mathbf{l}}\left(i m_{1}\right) \frac{1}{\left(q^{1 / k}\right)^{l_{0}\left(l_{0}-1\right) / 2}} \\
& \times \sigma_{q ; \tau}^{l_{1}-\left(l_{0} / k\right)}\left(\sum_{h=1}^{l_{2}} \frac{a_{h, l_{2}}}{\left(q^{l_{1}-\left(l_{0} / k\right)}\right)^{l_{0}}} \tau^{l_{0}+h}\left(\partial_{\tau}^{h} W\right)\left(\tau, m_{1}, \epsilon\right)\right) \mathrm{d} m_{1}+\Psi_{d}(\tau, m, \epsilon) .
\end{aligned}
$$


This last prepared shape allows us to apply the next lemma that repharses formula (8.7) p. 3630 from [19], in order to express all differential operators appearing in (72) in terms of the most basic one $\tau^{k^{\prime}+1} \partial_{\tau}$.

Lemma 7. Let $k^{\prime}, \delta \geq 1$ be integers. Then, there exist real numbers $A_{\delta, p}, 1 \leq p \leq \delta-1$, such that

$$
\tau^{\delta\left(k^{\prime}+1\right)} \partial_{\tau}^{\delta}=\left(\tau^{k^{\prime}+1} \partial_{\tau}\right)^{\delta}+\sum_{1 \leq p \leq \delta-1} A_{\delta, p} \tau^{k^{\prime}(\delta-p)}\left(\tau^{k^{\prime}+1} \partial_{\tau}\right)^{p} .
$$

By convention, we take for granted that the above sum $\sum_{1 \leq p \leq \delta-1}[.$.$] vanishes when \delta=1$.

Indeed, by construction of the finite set $I$, we can represent the next integers in a specific way:

$$
l_{0}+h=h\left(1+k^{\prime}\right)+e_{h, l_{0}}
$$

where $e_{h, l_{0}}=l_{0}-h k^{\prime} \geq 1$ for all $\left(l_{0}, l_{1}, l_{2}\right) \in I$ and $1 \leq h \leq l_{2}$. As a consequence, we can further expand the next piece of (72) in its final convenient form:

$$
\begin{aligned}
\tau^{l_{0}+h} \partial_{\tau}^{h} W\left(\tau, m_{1}, \epsilon\right) & =\tau^{e_{h, l_{0}}} \tau^{h\left(1+k^{\prime}\right)} \partial_{\tau}^{h} W\left(\tau, m_{1}, \epsilon\right) \\
& =\tau^{e_{h, l_{0}}}\left(\left(\tau^{k^{\prime}+1} \partial_{\tau}\right)^{h}+\sum_{1 \leq p \leq h-1} A_{h, p} \tau^{k^{\prime}(h-p)}\left(\tau^{k^{\prime}+1} \partial_{\tau}\right)^{p}\right) W\left(\tau, m_{1}, \epsilon\right),
\end{aligned}
$$

and we can remodel equation (72) in such a way that it contains only primitive building blocs:

$$
\begin{aligned}
Q(i m) W(\tau, m, \epsilon)= & R_{D}(i m) \frac{1}{\left(q^{1 / k}\right)^{m_{D} k^{\prime}\left(k^{\prime}-1\right) / 2}}\left(\tau^{k^{\prime}+1} \partial_{\tau}\right)^{m_{D}} W(\tau, m, \epsilon) \\
& +\sum_{l=\left(l_{0}, l_{1}, l_{2}\right) \epsilon I} \epsilon^{\Delta_{l}-l_{0}} \frac{1}{(2 \pi)^{1 / 2}} \int_{-\infty}^{+\infty} C_{l}\left(m-m_{1}, \epsilon\right) R_{l}\left(i m_{1}\right) \frac{1}{\left(q^{1 / k}\right)^{l_{0}\left(l_{0}-1\right) / 2}} \\
& \times \sigma_{q ; \tau}^{l_{1}-\left(l_{0} / k\right)}\left(\sum_{h=1}^{l_{2}} \frac{a_{h, l_{2}}}{\left(q^{l_{1}-\left(l_{0} / k\right)}\right)^{l_{0}}} \tau^{e_{h, l_{0}}}\left(\left(\tau^{k^{\prime}+1} \partial_{\tau}\right)^{h}+\sum_{1 \leq p \leq h-1} A_{h, p} \tau^{k^{\prime}(h-p)}\left(\tau^{k^{\prime}+1} \partial_{\tau}\right)^{p}\right) W\left(\tau, m_{1}, \epsilon\right)\right) \mathrm{d} m_{1} \\
& +\Psi_{d}(\tau, m, \epsilon) .
\end{aligned}
$$

Similarly to our previous technical Lemma 5, we disclose some useful commutation formulas dealing with the actions of the basic irregular operator $\tau^{k^{\prime}+1} \partial_{\tau}$, multiplication by monomials $\tau^{m^{\prime}}$, and of the $q$-difference operator $\sigma_{q ; \tau}$,

\section{Lemma 8}

(1) The action of the differential operators $\tau^{k^{\prime}+1} \partial_{\tau}$ on $W(\tau, m, \epsilon)$ is given by

$$
\begin{aligned}
\tau^{k^{\prime}+1} \partial_{\tau} W(\tau, m, \epsilon)= & k^{\prime} \int_{L_{\gamma}} k^{\prime} u^{k^{\prime}} w(u, m, \epsilon) \\
& \cdot \exp \left(-\left(\frac{u}{\tau}\right)^{k^{\prime}}\right) \frac{\mathrm{d} u}{u} .
\end{aligned}
$$

(2) Let $m^{\prime} \geq 1$ be an integer. The action of the multiplication by $\tau^{m^{\prime}}$ on $W(\tau, m, \epsilon)$ is described through the next formula:

$$
\begin{aligned}
\tau^{m^{\prime}} W(\tau, m, \epsilon)= & k^{\prime} \int_{L_{\gamma}}\left(\frac{u^{k^{\prime}}}{\Gamma\left(m^{\prime} / k^{\prime}\right)} \int_{0}^{u^{k^{\prime}}}\left(u^{k^{\prime}}-s\right)^{\left(m^{\prime} / k^{\prime}\right)-1}\right. \\
& \left.\cdot w\left(s^{1 / k^{\prime}}, m, \epsilon\right) \frac{\mathrm{d} s}{s}\right) \exp \left(-\left(\frac{u}{\tau}\right)^{k^{\prime}}\right) \frac{\mathrm{d} u}{u}
\end{aligned}
$$

(3) Let $\gamma \in \mathbb{Z}$ be an integer. The action of the operator $\sigma_{q ; \tau}^{\gamma}$ is represented through the following integral transform:

$$
\sigma_{q ; \tau}^{\gamma} W(\tau, m, \epsilon)=k^{\prime} \int_{L_{\gamma}} \sigma_{q ; u}^{\gamma} w(u, m, \epsilon) \exp \left(-\left(\frac{u}{\tau}\right)^{k^{\prime}}\right) \frac{\mathrm{d} u}{u} .
$$

Proof. The first two formulas have already been given in our previous works $[3,20]$. We focus on the third equality. By definition, 


$$
\sigma_{q ; \tau}^{\gamma} W(\tau, m, \epsilon)=k^{\prime} \int_{L_{\gamma}} w(u, m, \epsilon) \exp \left(-\left(\frac{u}{q^{\gamma} \tau}\right)^{k^{\prime}}\right) \frac{\mathrm{d} u}{u}
$$

and if one deforms the path of integration $L_{\gamma}$ through $u=$ $q^{\gamma} v$ which keeps the path invariant since $q^{\gamma} \in \mathbb{R}_{+}$, we get formula (79).

Departing from the arranged equation (76) with the help of Lemma 8 , we can exhibit an ancillary problem satisfied by the expression $w(u, m, \epsilon)$,

$$
\begin{aligned}
Q(i m) w(u, m, \epsilon)= & R_{D}(i m) \frac{\left(k^{\prime}\right)^{m_{D}}}{\left(q^{1 / k}\right)^{m_{D} k^{\prime}\left(k^{\prime}-1\right) / 2}} u^{k^{\prime} m_{D}} w(u, m, \epsilon) \\
& +\sum_{l=\left(l_{0}, l_{1}, l_{2}\right) \in I} \epsilon^{\Delta_{l}-l_{0}} \frac{1}{(2 \pi)^{1 / 2}} \int_{-\infty}^{+\infty} C_{l}\left(m-m_{1}, \epsilon\right) R_{1}\left(i m_{1}\right) \frac{1}{\left(q^{1 / k}\right)^{l_{0}\left(l_{0}-1\right) / 2} \sigma_{q ; u}^{l_{1}-\left(l_{0} / k\right)}} \\
& +\left\{\sum _ { h = 1 } ^ { l _ { 2 } } \frac { a _ { h , l _ { 2 } } } { ( q ^ { l _ { 1 } - ( l _ { 0 } / k ) } ) ^ { l _ { 0 } } } \left(\frac{u^{k^{\prime}}}{\Gamma\left(e_{h, l_{0}} / k^{\prime}\right)} \int_{0}^{u^{k^{\prime}}}\left(u^{k^{\prime}}-s\right)^{\left(e_{h, l_{0}} / k^{\prime}\right)-1}\left(k^{\prime}\right)^{h} s^{h} w\left(s^{1 / k^{\prime}}, m_{1}, \epsilon\right) \frac{\mathrm{d} s}{s}\right.\right. \\
& \left.\left.+\sum_{1 \leq p \leq h-1} A_{h, p} \frac{u^{k^{\prime}}}{\Gamma\left(\left(e_{h, l_{0}}+k^{\prime}(h-p)\right) / k^{\prime}\right)} \int_{0}^{u^{k^{\prime}}}\left(u^{k^{\prime}}-s\right)^{\left(\left(e_{h, l_{0}}+k^{\prime}(h-p)\right) / k^{\prime}\right)-1}\left(k^{\prime}\right)^{p} s^{p} w\left(s^{1 / k^{\prime}}, m_{1}, \epsilon\right) \frac{\mathrm{d} s}{s}\right)\right\} \mathrm{d} m_{1} \\
& +\psi(u, m, \epsilon) .
\end{aligned}
$$

\section{An Integral q-Difference Equation with Complex Parameter}

The objective of this section is the construction of a unique solution of equation (81) just established overhead. This solution will be built among the Banach space displayed in Definition 5. Within the next three propositions, continuity of linear convolutions and $q$-difference operators acting on $\operatorname{Exp}_{\left(k_{1}, \beta, \mu, \alpha, r\right)}^{q}$ is discussed.

Proposition 1. Let $k^{\prime} \geq 1$ be an integer and $\gamma_{1}>0$ and $\gamma_{2}, \gamma_{3}$ be real numbers such that $k^{\prime}\left(\gamma_{2}+\gamma_{3}+2\right)$ is an integer that are submitted to the next constraint:

$$
\begin{aligned}
& \gamma_{2}+1>0, \\
& \gamma_{3}+\frac{1}{k^{\prime}}+1>0, \\
& \gamma_{1} \geq k^{\prime}\left(\gamma_{2}+\gamma_{3}+2\right) \geq 0 .
\end{aligned}
$$

Let $a_{\gamma_{1}}(u)$ be a continuous function on $\left(\overline{U_{d}} \cup \overline{D(0, r)}\right)$, holomorphic w.r.t $u$ on $U_{d} \cup D(0, r)$ submitted to the bound

$$
\left|a_{\gamma_{1}}(u)\right| \leq \frac{M_{\gamma_{1}}}{(1+|u|)^{\gamma_{1}}}
$$

for all $u \in \overline{U_{d}} \cup \overline{D(0, r)}$, for some constant $M_{\gamma_{1}}>0$. Then, the linear function

$$
\begin{aligned}
f \longmapsto \mathscr{C}_{k^{\prime}, \gamma_{1}, \gamma_{2}, \gamma_{3}}(f)(u, m)= & a_{\gamma_{1}}(u) u^{k^{\prime}} \int_{0}^{u^{k^{\prime}}}\left(u^{k^{\prime}}-s\right)^{\gamma_{2}} s^{\gamma_{3}} \\
& \cdot f\left(s^{1 / k^{\prime}}, m\right) \mathrm{d} s,
\end{aligned}
$$

represents a continuous map from the Banach space $\operatorname{Exp}_{\left(k_{1}, \beta, \mu, \alpha, r\right)}^{q}$ into itself. In other words, some constant $M_{1}>0$ (depending on $k^{\prime}, \gamma_{1}, \gamma_{2}, \gamma_{3}$ ) can be found with

$$
\left\|\mathscr{C}_{k^{\prime}, \gamma_{1}, \gamma_{2}, \gamma_{3}}(f)(u, m)\right\|_{\left(k_{1}, \beta, \mu, \alpha, r\right)} \leq M_{1} M_{\gamma_{1}}\|f(u, m)\|_{\left(k_{1}, \beta, \mu, \alpha, r\right)} \text {, }
$$

for all $f \in \operatorname{Exp}_{\left(k_{1}, \beta, \mu, \alpha, r\right)}^{q}$.

Proof. Let $f$ belong to $\operatorname{Exp}_{\left(k_{1}, \beta, \mu, \alpha, r\right)}^{q}$. The map $\mathscr{C}_{k^{\prime}, \gamma_{1}, \gamma_{2}, \gamma_{3}}(f)(u, m)$ can be rewritten using the parametrization $s=u^{k^{\prime}} p$ for $0 \leq p \leq 1$, namely,

$$
\begin{aligned}
\mathscr{C}_{k^{\prime}, \gamma_{1}, \gamma_{2}, \gamma_{3}}(f)(u, m)= & a_{\gamma_{1}}(u) u^{k^{\prime}\left(\gamma_{2}+\gamma_{3}+2\right)} \\
& \cdot \int_{0}^{1}(1-p)^{\gamma_{2}} p^{\gamma_{3}} f\left(u p^{1 / k^{\prime}}, m\right) \mathrm{d} p,
\end{aligned}
$$

for all $u \in U_{d} \cup D(0, r)$, whenever $m \in \mathbb{R}$. By definition, the next upper bounds

$$
\begin{aligned}
|f(u, m)| \leq & \|f(u, m)\|_{\left(k_{1}, \beta, \mu, \alpha, r\right)}|u| \\
& \cdot \exp \left(\frac{k_{1}}{2} \frac{\log ^{2}(|u|+\delta)}{\log (q)}+\alpha \log (|u|+\delta)\right) \\
& \cdot(1+|m|)^{-\mu} e^{-\beta|m|},
\end{aligned}
$$




$$
\begin{aligned}
\left|\mathscr{C}_{k^{\prime}, \gamma_{1}, \gamma_{2}, \gamma_{3}}(f)(u, m)\right| \leq & \frac{M_{\gamma_{1}}|u|^{k^{\prime}\left(\gamma_{2}+\gamma_{3}+2\right)}}{(1+|u|)^{\gamma_{1}}} \int_{0}^{1}(1-p)^{\gamma_{2}} p^{\gamma_{3}+\left(1 / k^{\prime}\right)} \mathrm{d} p\|f(u, m)\|_{\left(k_{1}, \beta, \mu, \alpha, r\right)}|u| \\
& \times \exp \left(\frac{k_{1}}{2} \frac{\log ^{2}(|u|+\delta)}{\log (q)}+\alpha \log (|u|+\delta)\right)(1+|m|)^{-\mu} e^{-\beta|m|},
\end{aligned}
$$

for all $u \in U_{d} \cup D(0, r)$, all $m \in \mathbb{R}$. Under condition (82), the expected bound (85) follows.

Proposition 2. Let $a \in \mathbb{C}$ be a complex number, $\gamma_{1} \geq 0$ be an integer, and $\gamma_{2} \geq 0$ be a real number withstanding the following condition:

$$
k_{1} \gamma_{2} \geq \gamma_{1} \text {. }
$$

Then, we can sort a constant $M_{2}>0$ (depending on $\left.\gamma_{1}, \gamma_{2}, q, \alpha, \delta, r, a\right)$ with

$$
\left\|(u+a)^{\gamma_{1}} \sigma_{q ; u}^{-\gamma_{2}} f(u, m)\right\|_{\left(k_{1}, \beta, \mu, \alpha, r\right)} \leq M_{2}\|f(u, m)\|_{\left(k_{1}, \beta, \mu, \alpha, r\right)},
$$

for all $f \in \operatorname{Exp}_{\left(k_{1}, \beta, \mu, \alpha, r\right)}^{q}$.

Proof. The proof is proximate to the one of Proposition 1 in [5] and similar to the one of Proposition 1 from [18]. We provide, however, a complete proof for the sake of a better readability.

Let $f(u, m)$ belong to $\operatorname{Exp}_{\left(k_{1}, \beta, \mu, \alpha, r\right)}^{q}$. By definition, we can perform the next factorization

$$
\begin{aligned}
\left\|(u+a)^{\gamma_{1}} \sigma_{q ; u}^{-\gamma_{2}} f(u, m)\right\|_{\left(k_{1}, \beta, \mu, \alpha, r\right)}= & \sup _{u \in \bar{U}_{d}} \cup \overline{D(0, r)}(1+|m|)^{\mu} e^{\beta|m|} \frac{1}{|u|} \\
& \times \exp \left(-\frac{k_{1}}{2} \frac{\log ^{2}(|u|+\delta)}{\log (q)}-\alpha \log (|u|+\delta)\right)|u+a|^{\gamma_{1}} \\
& \times\left\{\left|f\left(\frac{u}{q^{\gamma_{2}}}, m\right)\right|(1+|m|)^{\mu} e^{\beta|m|} \frac{1}{\left|u / q^{\gamma_{2}}\right|} \exp \left(-\frac{k_{1}}{2} \frac{\log ^{2}\left(\left|u / q^{\gamma_{2}}\right|+\delta\right)}{\log (q)}-\alpha \log \left(\left|\frac{u}{q^{\gamma_{2}} \mid}\right|+\delta\right)\right)\right\} \\
& \times\left\{(1+|m|)^{-\mu} e^{-\beta|m|} \frac{|u|}{q^{\gamma_{2}}} \exp \left(\frac{k_{1}}{2} \frac{\log ^{2}\left(\left|u / q^{\gamma_{2}}\right|+\delta\right)}{\log (q)}+\alpha \log \left(\left|\frac{u}{q^{\gamma_{2}}}\right|+\delta\right)\right)\right\} .
\end{aligned}
$$

Since the contractive map $u \longmapsto u / q^{\gamma_{2}}$ keeps the domain $\overline{U_{d}} \cup \overline{D(0, r)}$ invariant, we deduce

$$
\left\|(u+a)^{\gamma_{1}} \sigma_{q ; u}^{-\gamma_{2}} f(u, m)\right\|_{\left(k_{1}, \beta, \mu, \alpha, r\right)} \leq \tilde{M}_{2}\|f(u, m)\|_{\left(k_{1}, \beta, \mu, \alpha, r\right)},
$$

with $\tilde{M}_{2}=\left(1 / q^{\gamma_{2}}\right) \sup _{u \in \overline{U_{d}}} \mathrm{\cup} \overline{D(0, r)} \mathscr{A}(u)$, where

$$
\begin{aligned}
\mathscr{A}(u)= & |u+a|^{\gamma_{1}} \exp \left(-\frac{k_{1}}{2} \frac{\log ^{2}(|u|+\delta)}{\log (q)}-\alpha \log (|u|+\delta)\right) \\
& \times \exp \left(\frac{k_{1}}{2} \frac{\log ^{2}\left(\left|u / q^{\gamma_{2}}\right|+\delta\right)}{\log (q)}+\alpha \log \left(\left|\frac{u}{q^{\gamma_{2}}}\right|+\delta\right)\right) .
\end{aligned}
$$

We observe that

$$
\tilde{M}_{2} \leq \frac{1}{q^{\gamma_{2}}} \max \left(\tilde{M}_{2.1}, \tilde{M}_{2.2}\right),
$$

where $\tilde{M}_{2.1}=\sup _{u \in \overline{D(0, r)}} \mathscr{A}(u)$ is finite since $\mathscr{A}(u)$ is continuous on $\overline{D(0, r)}$ and $\widetilde{M}_{2.2}$ is equal to $\sup _{u \in \overline{U_{d}}}|u|>r \mathscr{A}(u)$.

In the remaining part of the proof, we show that $\tilde{M}_{2.2}$ is also finite. We first need to rearrange the pieces of $\mathscr{A}(u)$. Namely, we expand

$$
\begin{aligned}
\log ^{2}(|u|+\delta)= & \log ^{2}|u|+2 \log |u| \log \left(1+\frac{\delta}{|u|}\right) \\
& +\log ^{2}\left(1+\frac{\delta}{|u|}\right) \\
\log ^{2}\left(\left|\frac{u}{q^{\gamma_{2}}}\right|+\delta\right)= & \log ^{2}|u|-2 \gamma_{2} \log (q) \log |u|+\left(\gamma_{2} \log (q)\right)^{2} \\
& +2 \log \left|\frac{u}{q^{\gamma_{2}}}\right| \log \left(1+\frac{q^{\gamma_{2}}}{|u|} \delta\right)+\log ^{2}\left(1+\frac{q^{\gamma_{2}}}{|u|} \delta\right),
\end{aligned}
$$




$$
\begin{aligned}
\log (|u|+\delta) & =\log |u|+\log \left(1+\frac{\delta}{|u|}\right) \\
\log \left(\left|\frac{u}{q^{\gamma_{2}}}\right|+\delta\right) & =\log |u|-\gamma_{2} \log (q)+\log \left(1+\frac{q^{\gamma_{2}}}{|u|} \delta\right) .
\end{aligned}
$$

Since $\log (1+x) \sim x$ as $x \longrightarrow 0$, we get two constants $A_{1}, A_{2} \in \mathbb{R}$ (depending on $r, \delta, q, \gamma_{2}$ ) with

$$
\begin{aligned}
& A_{1} \leq 2 \log |u| \log \left(1+\frac{\delta}{|u|}\right) \leq A_{2}, \\
& A_{1} \leq 2 \log \left|\frac{u}{q^{\gamma_{2}}}\right| \log \left(1+\frac{q^{\gamma_{2}}}{|u|} \delta\right) \leq A_{2},
\end{aligned}
$$

for all $u \in \overline{U_{d}},|u|>r$. Gathering (95) and (96) with (97) gives rise to the bound

$$
\begin{aligned}
\tilde{M}_{2.2} \leq & \left(\operatorname { s u p } _ { u \in \overline { U } _ { d } , | u | > r } \frac { | u + a | ^ { \gamma _ { 1 } } } { | u | ^ { \gamma _ { 2 } k _ { 1 } } } \operatorname { e x p } \left(\frac{k_{1} \gamma_{2}^{2}}{2} \log (q)+\frac{A_{2} k_{1}}{2 \log (q)}+\frac{k_{1}}{2 \log (q)} \log ^{2}\left(1+\frac{q^{\gamma_{2}}}{r} \delta\right)\right.\right. \\
& -A_{1} \frac{k_{1}}{2 \log (q)}-\alpha \gamma_{2} \log (q)+\alpha \log \left(1+\frac{q^{\gamma_{2}}}{r} \delta\right),
\end{aligned}
$$

which is finite owing to (89).

Proposition 3. We set polynomials $Q(X), R(X) \in \mathbb{C}[X]$ such that

$$
\begin{aligned}
\operatorname{deg}(R) & \geq \operatorname{deg}(Q), \\
R(i m) & \neq 0, \\
\mu & >\operatorname{deg}(Q)+1 .
\end{aligned}
$$

Consider $(u, m) \longmapsto b(u, m)$ a continuous function on $\left(\overline{U_{d}} \cup \overline{D(0, r)}\right) \times \mathbb{R}$, holomorphic w.r.t u on $U_{d} \cup D(0, r)$ with the bound

$$
\sup _{\substack{u \in \overline{U_{d}} \cup \overline{D(0, r)} \\ m \in \mathbb{R}}}|b(u, m)| \leq M_{b}
$$

for some constant $M_{b}>0$. Then, there exists a constant $M_{3}>0$ (depending on $Q, R$, and $\mu$ ) such that

$$
\begin{aligned}
& \left\|\frac{1}{R(i m)} \int_{-\infty}^{+\infty} f\left(m-m_{1}\right) Q\left(i m_{1}\right) b(u, m) g\left(u, m_{1}\right) \mathrm{d} m_{1}\right\|_{\left(k_{1}, \beta, \mu, \alpha, r\right)} \\
& \quad \leq M_{3} M_{b}\|f(m)\|_{(\beta, \mu)}\|g(u, m)\|_{\left(k_{1}, \beta, \mu, \alpha, r\right)},
\end{aligned}
$$

whenever $f$ belongs to $E_{(\beta, \mu)}$ and $g$ belongs to $\operatorname{Exp}_{\left(k_{1}, \beta, \mu, \alpha, r\right)}^{q}$.

Proof. The proof shares the same ingredients as the one of Proposition 2 of [3]. Again, we give a thorough explanation of the result. We take $f$ inside $E_{(\beta, \mu)}$ and select $g$ belonging to $\operatorname{Exp}_{\left(k_{1}, \beta, \mu, \alpha, r\right)}^{q}$. We first recast the norm of the convolution operator as follows:

$$
\begin{aligned}
N_{2}= & \left\|\frac{1}{R(i m)} \int_{-\infty}^{+\infty} f\left(m-m_{1}\right) Q\left(i m_{1}\right) b(u, m) g\left(u, m_{1}\right) \mathrm{d} m_{1}\right\|_{\left(k_{1}, \beta, \mu, \alpha, r\right)} \\
= & \sup _{u \in \overline{U_{d}} \cup \overline{D(0, r), m \in \mathbb{R}}}(1+|m|)^{\mu} \frac{1}{|u|} e^{\beta|m|} \exp \left(-\frac{k_{1}}{2} \frac{\log ^{2}(|u|+\delta)}{\log (q)}-\alpha \log (|u|+\delta)\right) \\
& \times \mid \frac{1}{R(i m)} \int_{-\infty}^{+\infty}\left\{\left(1+\left|m-m_{1}\right|\right)^{\mu} \exp \left(\beta\left|m-m_{1}\right|\right) f\left(m-m_{1}\right)\right\} b(u, m) \\
& \times\left\{\left(1+\left|m_{1}\right|\right)^{\mu} e^{\beta\left|m_{1}\right|} \frac{1}{|u|} \exp \left(-\frac{k_{1}}{2} \frac{\log ^{2}(|u|+\delta)}{\log (q)}-\alpha \log (|u|+\delta)\right) g\left(u, m_{1}\right)\right\} \mathscr{A}\left(u, m, m_{1}\right) \mathrm{d} m_{1} \mid,
\end{aligned}
$$

where

$$
\mathscr{A}\left(u, m, m_{1}\right)=\frac{Q\left(i m_{1}\right) \exp \left(-\beta\left|m_{1}\right|\right) \exp \left(-\beta\left|m-m_{1}\right|\right)}{\left(1+\left|m_{1}\right|\right)^{\mu}\left(1+\left|m-m_{1}\right|\right)^{\mu}}|u| \exp \left(\frac{k_{1}}{2} \frac{\log ^{2}(|u|+\delta)}{\log (q)}+\alpha \log (|u|+\delta)\right) .
$$


By construction of the polynomials $Q$ and $R$, one can sort two constants $\mathbb{Q}, \mathfrak{R}>0$ with

$$
\begin{gathered}
\left|Q\left(i m_{1}\right)\right| \leq Q\left(1+\left|m_{1}\right|\right)^{\operatorname{deg}(Q)}, \\
|R(i m)| \geq \Re(1+|m|)^{\operatorname{deg}(R)},
\end{gathered}
$$

for all $m, m_{1} \in \mathbb{R}$. As a consequence of (102), (104), and (100), with the help of the triangular inequality $|m| \leq\left|m_{1}\right|+\left|m-m_{1}\right|$, we are led to the bounds

$$
N_{2} \leq M_{3} M_{b}\|f(m)\|_{(\beta, \mu)}\|g(u, m)\|_{\left(k_{1}, \beta, \mu, \alpha, r\right)},
$$

where

$$
\begin{aligned}
M_{3}= & \frac{Q}{\mathfrak{R}} \sup _{m \in \mathbb{R}}(1+|m|)^{\mu-\operatorname{deg}(R)} \\
& \cdot \int_{-\infty}^{+\infty} \frac{1}{\left(1+\left|m-m_{1}\right|\right)^{\mu}\left(1+\left|m_{1}\right|\right)^{\mu-\operatorname{deg}(Q)}} \mathrm{d} m_{1},
\end{aligned}
$$

is a finite constant under the first and last restriction of (99) according to the estimates of Lemma 2.2 from [21] or Lemma 4 of [22].

We disclose now additional assumptions on the leading polynomials $\mathrm{Q}(X)$ and $R_{D}(X)$. These requirements will be essential in the transformation of our main problem (81) into a fixed point equation, as explained later in Proposition 4.
With this respect, the guideline is close to our previous study [3]. Namely, we assume the existence of an unbounded sectorial annulus:

$$
S_{Q, R_{D}}=\left\{z \in \frac{\mathbb{C}^{*}}{|z|}>r_{Q, R_{D}},\left|\arg (z)-d_{Q, R_{D}}\right| \leq \eta_{Q, R_{D}}\right\},
$$

where direction $d_{Q, R_{D}} \in \mathbb{R}$ and aperture $\eta_{Q, R_{D}}>0$ for some given inner radius $r_{Q, R_{D}}>0$ with the feature:

$$
\left\{\frac{Q(i m) / R_{D}(i m)}{m} \in \mathbb{R}\right\} \subset S_{Q, R_{D}} \text {. }
$$

We consider the next polynomial:

$$
P_{m}(u)=Q(i m)-R_{D}(i m) \frac{\left(k^{\prime}\right)^{m_{D}}}{\left(q^{1 / k}\right)^{m_{D} k^{\prime}\left(k^{\prime}-1\right) / 2}} u^{k^{\prime} m_{D}} .
$$

In the following, we need lower bounds of the expression $P_{m}(u)$ with respect to both variables $m$ and $u$. In order to achieve this goal, we can factorize the polynomial w.r.t $u$, namely,

$$
P_{m}(u)=-R_{D}(i m) \frac{\left(k^{\prime}\right)^{m_{D}}}{\left(q^{1 / k}\right)^{m_{D} k^{\prime}\left(k^{\prime}-1\right) / 2}} \prod_{l=0}^{k^{\prime} m_{D}-1}\left(u-q_{l}(m)\right),
$$

where its roots $q_{l}(m)$ can be displayed explicitely as

$$
q_{l}(m)=\left(\frac{|Q(i m)|}{\left|R_{D}(i m)\right|\left(\left(k^{\prime}\right)^{m_{D}} /\left(q^{1 / k}\right)^{m_{D} k^{\prime}\left(k^{\prime}-1\right) / 2}\right)}\right)^{1 / k^{\prime} m_{D}} \exp \left(\frac{\sqrt{-1}}{k^{\prime} m_{D}}\left(\arg \left(\frac{Q(i m)}{R_{D}(i m)\left(\left(k^{\prime}\right)^{m_{D}} /\left(q^{1 / k}\right)^{m_{D} k^{\prime}\left(k^{\prime}-1\right) / 2}\right)}\right)+2 \pi l\right)\right),
$$

for all $0 \leq l \leq k^{\prime} m_{D}-1$, for all $m \in \mathbb{R}$.

We set an unbounded sector $U_{d}$ centered at 0 , a small disc $D(0, r)$, and we adjust the sector $S_{Q, R_{D}}$ in a way that the next condition holds. A constant $\mathscr{M}>0$ can be chosen with

$$
\left|u-q_{l}(m)\right| \geq \mathscr{M}(1+|u|),
$$

for all $0 \leq l \leq k^{\prime} m_{D}-1$, all $m \in \mathbb{R}$, provided that $u \in U_{d} \cup D(0, r)$. Indeed, inclusion (108) implies in particular that all the roots $q_{l}(m), 0 \leq l \leq k^{\prime} m_{D}-1$ remain a part of some neighborhood of the origin, i.e., satisfy $\left|q_{l}(m)\right| \geq 2 r$ for an appropriate choice of $r>0$. Furthermore, when the aperture $\eta_{Q, R_{D}}>0$ is taken close enough to 0 , all these roots $q_{l}(m)$ stay inside a union $\mathcal{U}$ of unbounded sectors centered at 0 that do not cover a full neighborhood of 0 in $\mathbb{C}^{*}$. We assign a sector $U_{d}$ with

$$
U_{d} \cap \mathscr{U}=\varnothing .
$$

By construction, the quotients $q_{l}(m) / u$ live outside some small disc centered at 1 in $\mathbb{C}$ for all $u \in U_{d}, m \in \mathbb{R}$, $0 \leq l \leq k^{\prime} m_{D}-1$. Then, (112) follows.

We are now ready to supply lower bounds for $P_{m}(u)$.
Lemma 9. A constant $C_{P}>0$ (depending on $k, k^{\prime}, m_{D}, q, \mathscr{M}$ ) can be found with

$$
\left|P_{m}(u)\right| \geq C_{P}\left|R_{D}(i m)\right|(1+|u|)^{k^{\prime} m_{D}},
$$

for $u \in U_{d} \cup D(0, r)$, all $m \in \mathbb{R}$.

Proof. Departing from factorization (110), the lower bound (112) entails

$$
\left|P_{m}(u)\right| \geq \frac{\left(k^{\prime}\right)^{m_{D}}}{\left(q^{1 / k}\right)^{m_{D} k^{\prime}\left(k^{\prime}-1\right) / 2}} \mathscr{M}^{k^{\prime} m_{D}}\left|R_{D}(i m)\right|(1+|u|)^{k^{\prime} m_{D}}
$$

for all $u \in U_{d} \cup D(0, r)$.

The next proposition discusses sufficient conditions under which a solution $w^{d}(u, m, \epsilon)$ of the main integral $q$ difference equation (81) can be built up in the space $\operatorname{Exp}_{\left(k_{1}, \beta, \mu, \alpha, r\right)}^{q}$.

Proposition 4. Let us assume the next extra requirements: 


$$
\begin{aligned}
\frac{l_{0}}{k}-l_{1} & \geq 0, \\
\mu & >\operatorname{deg}\left(R_{1}\right),
\end{aligned}
$$

for all $l=\left(l_{0}, l_{1}, l_{2}\right) \in I$. Furthermore, for each $l=\left(l_{0}, l_{1}, l_{2}\right) \in I$, we set an integer $p_{l_{0}, l_{1}}$ such that

$$
0 \leq p_{l_{0}, l_{1}} \leq k_{1}\left(\frac{l_{0}}{k}-l_{1}\right)
$$

and we take for granted that

$$
p_{l_{0}, l_{1}}+k^{\prime} m_{D} \geq l_{0}
$$

holds. Then, for an appropriate choice of the constants $C_{l}>0$ (see (34)) that need to be taken close enough to 0 for all $l \in I, a$ constant $₫>0$ can be singled out in a manner that equation (81) gets a unique solution $(u, m) \longmapsto w^{d}(u, m, \epsilon)$ in the space $\operatorname{Exp}_{\left(k_{1}, \beta, \mu, \alpha, r\right)}^{q}$ with the condition:

$$
\left\|w^{d}(u, m, \epsilon)\right\|_{\left(k_{1}, \beta, \mu, \alpha, r\right)} \leq \emptyset,
$$

whenever $\epsilon \in D\left(0, \epsilon_{0}\right)$, where $U_{d}, r$ are chosen as above and $k_{1}, \beta, \mu, \alpha$ are specified in Section 3 on the way to the construction of the forcing term $f(t, z, \epsilon)$.

Proof. The proof relies strongly on the next lemma which discusses contractive properties of a linear map.

Lemma 10. For all $\epsilon \in D\left(0, \epsilon_{0}\right)$, we define the map $\mathscr{H}_{\epsilon}$ as

$$
\begin{aligned}
& \mathscr{H}_{\epsilon}(w(u, m)):=\sum_{l=\left(l_{0}, l_{1}, l_{2}\right) \in I} \epsilon^{\Delta_{l}-l_{0}} \frac{1}{(2 \pi)^{1 / 2}} \\
& \cdot \int_{-\infty}^{+\infty} C_{l}\left(m-m_{1}, \epsilon\right) R_{l}\left(i m_{1}\right) \frac{1}{\left(q^{1 / k}\right)^{l_{0}\left(l_{0}-1\right) / 2}} \frac{1}{P_{m}(u)} \sigma_{q ; u}^{l_{1}-\left(l_{0} / k\right)} \\
& \left\{\sum _ { h = 1 } ^ { l _ { 2 } } \frac { a _ { h , l _ { 2 } } } { ( q ^ { l _ { 1 } - ( l _ { 0 } / k ) } ) ^ { l _ { 0 } } } \left(\frac{u^{k^{\prime}}}{\Gamma\left(e_{h, l_{0}} / k^{\prime}\right)} \int_{0}^{u^{k^{\prime}}}\left(u^{k^{\prime}}-s\right)^{\left(e_{h, l_{0}} / k^{\prime}\right)-1}\left(k^{\prime}\right)^{h} s^{h} w\left(s^{1 / k^{\prime}}, m_{1}\right) \frac{\mathrm{d} s}{s}\right.\right. \\
& \left.\left.+\sum_{1 \leq p \leq h-1} A_{h, p} \frac{u^{k^{\prime}}}{\Gamma\left(\left(e_{h, l_{0}}+k^{\prime}(h-p)\right) / k^{\prime}\right)} \int_{0}^{u^{k^{\prime}}}\left(u^{k^{\prime}}-s\right)^{\left(\left(e_{h, l_{0}}+k^{\prime}(h-p)\right) / k^{\prime}\right)-1}\left(k^{\prime}\right)^{p} s^{p} w\left(s^{1 / k^{\prime}}, m_{1}\right) \frac{\mathrm{d} s}{s}\right)\right\} \mathrm{d} m_{1} \\
& +\frac{\psi(u, m, \epsilon)}{P_{m}(u)} .
\end{aligned}
$$

Under the additional requirement (116)-(118), one can select the constants $C_{l}>0$, for $l \in I$, and a real number $\omega>0$ in a way that this map acts on some neighborhood of the origin of the space $\operatorname{Exp}_{\left(k_{1}, \beta, \mu, \alpha, r\right)}^{q}$ in the following way:

(i) The inclusion

$$
\mathscr{H}_{\epsilon}(\overline{B(0, \Phi)}) \subset \overline{B(0, \Phi)},
$$

holds, where $\overline{B(0, \Phi)}$ stands for the closed ball of radius $\Phi$ centered at 0 in $\operatorname{Exp}_{\left(k_{1}, \beta, \mu, \alpha, r\right)}^{q}$, for all $\epsilon \in D\left(0, \epsilon_{0}\right)$.

(ii) The map $\mathscr{H}_{\epsilon}$ is contractive, namely,

$$
\begin{gathered}
\left\|\mathscr{H}_{\epsilon}\left(w_{2}(u, m)\right)-\mathscr{H}_{\epsilon}\left(w_{1}(u, m)\right)\right\|_{\left(k_{1}, \beta, \mu, \alpha, r\right)} \\
\leq\left\|\frac{1}{2} w_{2}(u, m)-w_{1}(u, m)\right\|_{\left(k_{1}, \beta, \mu, \alpha, r\right)},
\end{gathered}
$$

$$
\text { whenever } w_{1}, w_{2} \in \bar{B}(0, \Phi) \text {, for all } \epsilon \in D\left(0, \epsilon_{0}\right) \text {. }
$$

Proof. We first control the forcing term. Owing to bound (76) in Lemma 4, together with (114), we can exhibit a constant $C_{1}>0$ (relying on $q, k_{1}, \alpha, T_{0}$ ) with

$$
\left\|\frac{\psi(u, m, \epsilon)}{P_{m}(u)}\right\|_{\left(k_{1}, \beta, \mu, \alpha, r\right)} \leq \frac{K_{0} C_{1}}{C_{P} \min _{m \in \mathbb{R}}\left|R_{D}(i m)\right|},
$$

where $K_{0}>0$ is a constant that is set in (36), whenever $\epsilon \in D\left(0, \epsilon_{0}\right)$.

We deal with the first property (121). Let us take $w(\tau, m)$ in $\operatorname{Exp}_{\left(k_{1}, \beta, \mu, \alpha, r\right)}^{q}$ under the constraint $\|w(\tau, m)\|_{\left(k_{1}, \beta, \mu, \alpha, r\right)} \leq \boldsymbol{\omega}$.

We fix some complex number $\omega_{d}$ such that $\omega_{d} \notin \overline{U_{d}} \cup \overline{D(0, r)}$, and we redraft the norm of the next integral expression as follows: 


$$
\begin{aligned}
I_{1}= & \left\|\int_{-\infty}^{+\infty} C_{\mathbf{l}}\left(m-m_{1}, \epsilon\right) R_{\mathbf{l}}\left(i m_{1}\right) \frac{1}{P_{m}(u)} \times \sigma_{q ; u}^{l_{1}-\left(l_{0} / k\right)}\left(u^{k^{\prime}} \int_{0}^{u^{k^{\prime}}}\left(u^{k^{\prime}}-s\right)^{\left(e_{h, l_{0}} / k^{\prime}\right)-1} s^{h-1} w\left(s^{1 / k^{\prime}}, m_{1}\right) \mathrm{d} s\right) \mathrm{d} m_{1}\right\|_{\left(k_{1}, \beta, \mu, \alpha, r\right)} \\
= & \| \frac{1}{R_{D}(i m)} \int_{-\infty}^{+\infty} C_{\mathbf{l}}\left(m-m_{1}, \epsilon\right) R_{\mathbf{l}}\left(i m_{1}\right) \mathscr{B}(u, m)\left(u-\omega_{d}\right)^{p_{l_{0}, l_{1}}} \sigma_{q ; u}^{-\left(\left(l_{0} / k\right)-l_{1}\right)}\left(\frac{u^{k^{\prime}}}{\left(u-\omega_{d}\right)^{l_{0}}}\right. \\
& \left.\times \int_{0}^{u^{k^{\prime}}}\left(u^{k^{\prime}}-s\right)^{\left(e_{h, l_{0}} / k^{\prime}\right)-1} s^{h-1} w\left(s^{1 / k^{\prime}}, m_{1}\right) \mathrm{d} s\right) \mathrm{d} m_{1} \|_{\left(k_{1}, \beta, \mu, \alpha, r\right)},
\end{aligned}
$$

for all $l \in I, 1 \leq h \leq l_{2}$, where $p_{l_{0}, l_{1}} \geq 0$ is an integer chosen as in (117) and

$$
\mathscr{B}(u, m)=\frac{\left(q^{-\left(\left(l_{0} / k\right)-l_{1}\right)} u-\omega_{d}\right)^{l_{0}} R_{D}(i m)}{\left(u-\omega_{d}\right)^{p_{l_{0}, l_{1}}} P_{m}(u)} .
$$

We observe that a constant $M_{\mathscr{B}}>0$ (depending on $\left.q, l_{0}, l_{1}, p_{l_{0}, l_{1}}, k, k^{\prime}, m_{D}, \omega_{d}, \mathscr{M}\right)$ can be picked up with

$$
|\mathscr{B}(u, m)| \leq M_{\mathscr{B}},
$$

for all $u \in U_{d} \cup D(0, r), m \in \mathbb{R}$. Indeed, from (114), we obtain

$$
|\mathscr{B}(u, m)| \leq \frac{1}{C_{P}} \sup _{u \in \overline{U_{d}}} \cup \overline{D(0, r)} \frac{\left|q^{-\left(\left(l_{0} / k\right)-l_{1}\right)} u-\omega_{d}\right|^{l_{0}}}{\left|u-\omega_{d}\right|^{p_{l_{0}, l_{1}}}(1+|u|)^{k^{\prime} m_{D}}},
$$

for all $u \in U_{d} \cup D(0, r), m \in \mathbb{R}$ where the right-hand side is finite owing to the suitable choices of $\omega_{d}$ and $p_{l_{0}, l_{1}}$ in (118).

Under requirements (32) and (116), an application of Proposition 3 yields a constant $M_{3.1}>0$ (depending on $R_{D}, R_{1}$ and $\mu$ ) such that

$$
I_{1} \leq M_{3.1} M_{\mathscr{B}}\left\|C_{l}(m, \epsilon)\right\|_{(\beta, \mu)} I_{2},
$$

where

$$
I_{2}=\left\|\left(u-\omega_{d}\right)^{p_{l_{0}, l_{1}}} \sigma_{q ; u}^{-\left(\left(l_{0} / k\right)-l_{1}\right)}\left(\frac{u^{k^{\prime}}}{\left(u-\omega_{d}\right)^{l_{0}}} \int_{0}^{u^{k^{\prime}}}\left(u^{k^{\prime}}-s\right)^{\left(e_{h, l_{0}} / k^{\prime}\right)-1} s^{h-1} w\left(s^{1 / k^{\prime}}, m\right) \mathrm{d} s\right)\right\|_{\left(k_{1}, \beta, \mu, \alpha, r\right)} .
$$

Conditions (116) and (117) allow us to call back Proposition 2 in order to get a constant $M_{2.1}>0$ (depending on $\left.l_{0}, l_{1}, p_{l_{0}, l_{1}}, k, q, \alpha, \delta, r, \omega_{d}\right)$ with

$$
I_{2} \leq M_{2.1} I_{3}
$$

where

$$
I_{3}=\left\|\frac{u^{k^{\prime}}}{\left(u-\omega_{d}\right)^{l_{0}}} \int_{0}^{u^{k^{\prime}}}\left(u^{k^{\prime}}-s\right)^{\left(e_{h, l_{0} / k^{\prime}}\right)-1} s^{h-1} w\left(s^{1 / k^{\prime}}, m\right) \mathrm{d} s\right\|_{\left(k_{1}, \beta, \mu, \alpha, r\right)} .
$$

Lastly, Proposition 1 gives rise to constants $M_{\omega_{d}, l_{0}}>0$ (depending on $\omega_{d}, l_{0}$ ) and $M_{1.1}>0$ (depending on $k^{\prime}, l_{0}, l_{2}$ ) with

$$
I_{3} \leq M_{1.1} M_{\omega_{d}, l_{0}}\|w(u, m)\|_{\left(k_{1}, \beta, \mu, \alpha, r\right)}
$$

By compiling (128)-(132), we obtain

$$
I_{1} \leq M_{1.1} M_{2.1} M_{3.1} M_{\mathscr{B}} M_{\omega_{d}, l_{0}}\left\|C_{l}(m, \epsilon)\right\|_{(\beta, \mu)}\|w(u, m)\|_{\left(k_{1}, \beta, \mu, \alpha, r\right)},
$$

for all $l \in I, 1 \leq h \leq l_{2}$.

We now turn to the second principal pieces of $\mathscr{H}_{\epsilon}$. Following the same lines of arguments as above, we obtain that

$$
\begin{aligned}
J_{1} & =\left\|\int_{-\infty}^{+\infty} C_{l}\left(m-m_{1}, \epsilon\right) R_{l}\left(i m_{1}\right) \frac{1}{P_{m}(u)} \times \sigma_{q ; u}^{l_{1}-\left(l_{0} / k\right)}\left(u^{k^{\prime}} \int_{0}^{u^{k^{\prime}}}\left(u^{k^{\prime}}-s\right){ }^{\left(\left(e_{h, l_{0}}+k^{\prime}(h-p)\right) / k^{\prime}\right)-1} s^{p-1} w\left(s^{1 / k^{\prime}}, m_{1}\right) \mathrm{d} s\right) \mathrm{d} m_{1}\right\|_{\left(k_{1}, \beta, \mu, \alpha, r\right)} \\
& \leq M_{2.1} M_{3.1} M_{\mathscr{B}}\left\|C_{l}(m, \epsilon)\right\|_{(\beta, \mu)} J_{3},
\end{aligned}
$$


where

$$
J_{3}=\left\|\frac{u^{k^{\prime}}}{\left(u-\omega_{d}\right)^{l_{0}}} \int_{0}^{u^{k^{\prime}}}\left(u^{k^{\prime}}-s\right)^{\left(\left(e_{h, l_{0}}+k^{\prime}(h-p)\right) / k^{\prime}\right)-1} s^{p-1} w\left(s^{1 / k^{\prime}}, m\right) \mathrm{d} s\right\|_{\left(k_{1}, \beta, \mu, \alpha, r\right)},
$$

for all $l \in I, 2 \leq h \leq l_{2}$ and $1 \leq p \leq h-1$. In order to give bounds for $J_{3}$, we make use of Proposition 1 which affords a constant $M_{1.2}>0$ (depending on $k^{\prime}, l_{0}, l_{2}$ ) with

$$
J_{3} \leq M_{1.2} M_{\omega_{d}, l_{0}}\|w(u, m)\|_{\left(k_{1}, \beta, \mu, \alpha, r\right)} .
$$

By combining (134) and (136), we obtain

$$
J_{1} \leq M_{1.2} M_{2.1} M_{3.1} M_{\mathscr{B}} M_{\omega_{d}, l_{0}}\left\|C_{l}(m, \epsilon)\right\|_{(\beta, \mu)}\|w(u, m)\|_{\left(k_{1}, \beta, \mu, \alpha, r\right)},
$$

for all $l \in I, 2 \leq h \leq l_{2}$ and $1 \leq p \leq h-1$.

In the next step, we impose the constants $C_{1}>0,1 \in I$, to stay close enough to 0 in order that a constant $\omega>0$ can be singled out with

$$
\begin{aligned}
& \sum_{l=\left(l_{0}, l_{1}, l_{2}\right) \in I} \epsilon_{0}^{\Delta_{l}-l_{0}} \frac{1}{(2 \pi)^{1 / 2}} \frac{1}{\left(q^{1 / k}\right)^{l_{0}\left(l_{0}-1\right) / 2}} \sum_{h=1}^{l_{2}} \frac{\left|a_{h, l_{2}}\right|}{\left(q^{l_{1}-\left(l_{0} / k\right)}\right)^{l_{0}}} \\
& \quad \times\left(\frac{\left(k^{\prime}\right)^{h}}{\Gamma\left(e_{h, l_{0}} / k^{\prime}\right)} M_{1.1} M_{2.1} M_{3.1} M_{\mathscr{B}} M_{\omega_{d}, l_{0}} C_{l} \Phi+\sum_{1 \leq p \leq h-1}\left|A_{h, p}\right| \frac{\left(k^{\prime}\right)^{p}}{\Gamma\left(\left(e_{h, l_{0}}+k^{\prime}(h-p)\right) / k^{\prime}\right)} M_{1.2} M_{2.1} M_{3.1} M_{\mathscr{B}} M_{\omega_{d}, l_{0}} C_{l} \Phi\right) \\
& +\frac{K_{0} C_{1}}{C_{P} \min _{m \in \mathbb{R}}\left|R_{D}(i m)\right|} \leq \omega .
\end{aligned}
$$

The collection of (123), 133 and (137) submitted to condition (138) yields the inclusion (121).

The next part of the proof is devoted to the explanation of the contractive property (122). Indeed, consider two functions $w_{1}(u, m)$ and $w_{2}(u, m)$ inside the ball $\overline{B(0, \Phi)} \subset \operatorname{Exp}_{\left(k_{1}, \beta, \mu, \alpha, r\right)}^{q}$. Then, an application of the two inequalities (133) and (137) overhead leads to

$$
\begin{aligned}
& \left\|\int_{-\infty}^{+\infty} C_{l}\left(m-m_{1}, \epsilon\right) R_{l}\left(i m_{1}\right) \frac{1}{P_{m}(u)} \times \sigma_{q ; u}^{l_{1}-l_{0} / k}\left(u^{k^{\prime}} \int_{0}^{u^{k^{\prime}}}\left(u^{k^{\prime}}-s\right)^{\left(e_{h, l_{0}} / k^{\prime}\right)-1} s^{h-1}\left(w_{2}\left(s^{1 / k^{\prime}}, m_{1}\right)-w_{1}\left(s^{1 / k^{\prime}}, m_{1}\right)\right) \mathrm{d} s\right) \mathrm{d} m_{1}\right\|_{\left(k_{1}, \beta, \mu, \alpha, r\right)} \\
& \quad \leq M_{1.1} M_{2.1} M_{3.1} M_{\mathscr{B}} M_{\omega_{d}, l_{0}}\left\|C_{l}(m, \epsilon)\right\|_{(\beta, \mu)}\left\|w_{2}(u, m)-w_{1}(u, m)\right\|_{\left(k_{1}, \beta, \mu, \alpha, r\right)}
\end{aligned}
$$

for all $l \in I, 1 \leq h \leq l_{2}$ and

$$
\begin{aligned}
& \left\|\int_{-\infty}^{+\infty} C_{l}\left(m-m_{1}, \epsilon\right) R_{l}\left(i m_{1}\right) \frac{1}{P_{m}(u)} \times \sigma_{q ; u}^{l_{1}-\left(l_{0} / k\right)}\left(u^{k^{\prime}} \int_{0}^{u^{k^{\prime}}}\left(u^{k^{\prime}}-s\right)^{\left(\left(e_{h, l_{0}}+k^{\prime}(h-p)\right) / k^{\prime}\right)-1} s^{p-1}\left(w_{2}\left(s^{1 / k^{\prime}}, m_{1}\right)-w_{1}\left(s^{1 / k^{\prime}}, m_{1}\right)\right) \mathrm{d} s\right) \mathrm{d} m_{1}\right\|_{\left(k_{1}, \beta, \mu, \alpha, r\right)} \\
& \leq M_{1.2} M_{2.1} M_{3.1} M_{\mathscr{B}} M_{\omega_{d}, l_{0}}\left\|C_{\mathbf{l}}(m, \epsilon)\right\|_{(\beta, \mu)}\left\|w_{2}(u, m)-w_{1}(u, m)\right\|_{\left(k_{1}, \beta, \mu, \alpha, r\right)},
\end{aligned}
$$

for all $l \in I, 2 \leq h \leq l_{2}$ and $1 \leq p \leq h-1$.

This time, we require the constants $C_{l}>0, l \in I$, to withstand the next inequality 


$$
\begin{aligned}
& \sum_{l=\left(l_{0}, l_{1}, l_{2}\right) \in I} \epsilon_{0}^{\Delta_{l}-l_{0}} \frac{1}{(2 \pi)^{1 / 2}} \frac{1}{\left(q^{1 / k}\right)^{l_{0}\left(l_{0}-1\right) / 2}} \sum_{h=1}^{l_{2}} \frac{\left|a_{h, l_{2}}\right|}{\left(q^{l_{1}-\left(l_{0} / k\right)}\right)^{l_{0}}} \\
& \quad \times\left(\frac{\left(k^{\prime}\right)^{h}}{\Gamma\left(e_{h, l_{0}} / k^{\prime}\right)} M_{1.1} M_{2.1} M_{3.1} M_{\mathscr{B}} M_{\omega_{d}, l_{0}} C_{l}+\sum_{1 \leq p \leq h-1}\left|A_{h, p}\right| \frac{\left(k^{\prime}\right)^{p}}{\Gamma\left(\left(e_{h, l_{0}}+k^{\prime}(h-p)\right) / k^{\prime}\right)} M_{1.2} M_{2.1} M_{3.1} M_{\mathscr{B}} M_{\omega_{d}, l_{0}} C_{l}\right) \\
& \leq \frac{1}{2} .
\end{aligned}
$$

Owing to (139) and (140), under demand (141), we obtain (122).

In conclusion, we choose the constants $C_{l}>0, l \in I$ in order that both (138) and (141) hold conjointly. This yield Lemma 10.

We go back to the core of Proposition 4. For $₫>0$, chosen as in the lemma above, we consider the closed ball $\overline{B(0, \Phi)} \subset \operatorname{Exp}_{\left(k_{1}, \beta, \mu, \alpha, r\right)}^{d}$ that stands for a complete metric space for the distance $d(x, y)=\|x-y\|_{\left(k_{1}, \beta, \mu, \alpha, r\right)}$. According to the same lemma, we observe that $\mathscr{H}_{\epsilon}$ induces a contractive application from $(\overline{B(0, \emptyset)}, d)$ into itself. Then, according to the classical contractive mapping theorem, the map $\mathscr{H}_{\epsilon}$ carries a unique fixed point that we set as $w^{d}(u, m, \epsilon)$; meaning that

$$
\mathscr{H}_{\epsilon}\left(w^{d}(u, m, \epsilon)\right)=w^{d}(u, m, \epsilon),
$$

that belongs to the ball $\overline{B(0, \emptyset)}$, for all $\epsilon \in D\left(0, \epsilon_{0}\right)$. Furthermore, the function $w^{d}(u, m, \epsilon)$ depends holomorphically on $\epsilon$ in $D\left(0, \epsilon_{0}\right)$. Let the term

$$
R_{D}(i m) \frac{\left(k^{\prime}\right)^{m_{D}}}{\left(q^{1 / k}\right)^{m_{D} k^{\prime}\left(k^{\prime}-1\right) / 2}} u^{k^{\prime} m_{D}} w(u, m, \epsilon),
$$

be taken from the right to the left-hand side of (81) and then divide by the polynomial $P_{m}(u)$ defined in (109). These operations allows (81) to be exactly recast into equation (142) above. Consequently, the unique fixed point $w^{d}(u, m, \epsilon)$ of $\mathscr{H}_{\epsilon}$ obtained overhead in $\bar{B}(0, \emptyset)$ precisely solves equation (81).

\section{An Integrodifferential $q$-Difference Equation with a Complex Parameter}

In this section, we build up a solution $W^{d}(\tau, m, \epsilon)$ to the integrodifferential $q$-difference equation (69) with the shape of a Laplace transform of order $k^{\prime}$ in direction $d$. Furthermore, we provide sharp bounds of this solution for large values of its $q$-Borel and Fourier variables $\tau$ and $m$.

Proposition 5. We depart from the solution $w^{d}(u, m, \epsilon)$ of the integral equation (81) that has just been constructed in Proposition 4. We define

$$
W^{d}(\tau, m, \epsilon)=k^{\prime} \int_{L_{\gamma}} w^{d}(u, m, \epsilon) \exp \left(-\left(\frac{u}{\tau}\right)^{k^{\prime}}\right) \frac{\mathrm{d} u}{u},
$$

as the Laplace transform of $w^{d}(u, m, \epsilon)$ of order $k^{\prime}$ in direction $d$, where the integration path $L_{\gamma}=\mathbb{R}_{+} e^{\sqrt{-1} \gamma}$ belongs to the sector $U_{d} \cup\{0\}$. Then, for all $\epsilon \in D\left(0, \epsilon_{0}\right)$, the map $(\tau, m, \epsilon) \longmapsto W^{d}(\tau, m, \epsilon)$ is continuous on a domain $S_{d} \times$ $\mathbb{R} \times D\left(0, \epsilon_{0}\right)$ and depends holomorphically on $(\tau, \epsilon)$ in $S_{d} \times D\left(0, \epsilon_{0}\right)$, where $S_{d}$ represents an unbounded sector with bisecting direction $d$ and opening $\theta_{k^{\prime}}$ that fulfills

$$
0<\theta_{k^{\prime}}<\frac{\pi}{k^{\prime}}+\operatorname{Ap}\left(U_{d}\right)
$$

for $A p\left(U_{d}\right)$ defined as the aperture of the sector $U_{d}$. Furthermore, the map $(\tau, m, \epsilon) \longmapsto W^{d}(\tau, m, \epsilon)$ withstands the next accurate bounds:

(1) One can single out three constants $E_{1}>0$ (depending on $\left.k^{\prime}, k_{1}, \delta, q, \alpha\right), E_{2}>1$ (depending on $k_{1}, k^{\prime}, q$ ), and $0<E_{3}<1$ (relying on $k^{\prime}, \theta_{k^{\prime}}, A p(U)$ ) such that

$$
\begin{aligned}
\left|W^{d}(\tau, m, \epsilon)\right| \leq & E_{1} \Subset(1+|m|)^{-\mu} e^{-\beta|m|} \exp \left(\frac{k_{1}}{2} \frac{\log ^{2}\left(|\tau| / E_{3}\right)}{\log (q)}+\left(\alpha+E_{2}\right) \log \left(|\tau| / E_{3}\right)\right) \\
& \times \exp \left\{\left(\frac{k_{1} \log \left(|\tau| / E_{3}\right)}{k^{\prime} \log (q)}+\frac{1}{2}\right) \log \left(\frac{k_{1} \log \left(|\tau| / E_{3}\right)}{k^{\prime} \log (q)}+1\right)\right\},
\end{aligned}
$$

for all $\tau \in S_{d}$ with $|\tau| \geq 2^{1 / k^{\prime}} E_{3} \delta, m \in \mathbb{R}$ and $\epsilon \in D\left(0, \epsilon_{0}\right)$.

(2) One can find a constant $E_{0}>0$ (depending on $\left.k^{\prime}, k_{1}, \delta, q, \alpha\right)$ with

$$
\left|W^{d}(\tau, m, \epsilon)\right| \leq E_{0} \bowtie(1+|m|)^{-\mu} e^{-\beta|m|},
$$

whenever $\tau \in S_{d}$ with $|\tau| \leq 2^{1 / k^{\prime}} E_{3} \delta$, all $m \in \mathbb{R}$, all $\epsilon \in D\left(0, \epsilon_{0}\right)$.

In particular, one can sort two constants $E_{4}, E_{5}>0$ (depending on $k^{\prime}, k_{1}, \delta, q, \alpha, \theta_{k^{\prime}}, A p\left(U_{d}\right)$ ) with 


$$
\begin{aligned}
\left|W^{d}(\tau, m, \epsilon)\right| \leq & E_{4} @(1+|m|)^{-\mu} e^{-\beta|m|} \\
& \cdot \exp \left(\frac{k_{2}}{2} \frac{\log ^{2}(|\tau|+\delta)}{\log (q)}+\left(\alpha+E_{5}\right) \log (|\tau|+\delta)\right),
\end{aligned}
$$

for all $\tau \in S_{d}, m \in \mathbb{R}$ and $\epsilon \in D\left(0, \epsilon_{0}\right)$, where $k_{1}<k_{2}<k$ was introduced in Section 3 just above (47).

Finally, $W^{d}(\tau, m, \epsilon)$ satisfies the first auxiliary integrodifferential q-difference equation (69) on the domain $S_{d} \times \mathbb{R} \times D\left(0, \epsilon_{0}\right)$.
Proof. Bound (119) in Proposition 4 can be recast as

$$
\begin{aligned}
\left|w^{d}(u, m, \epsilon)\right| \leq & (1+|m|)^{-\mu} e^{-\beta|m|}|u| \\
& \cdot \exp \left(\frac{k_{1}}{2} \frac{\log ^{2}(|u|+\delta)}{\log (q)}+\alpha \log (|u|+\delta)\right),
\end{aligned}
$$

which holds for all $(u, m, \epsilon) \in\left(U_{d} \cup D(0, r)\right) \times \mathbb{R} \times D\left(0, \epsilon_{0}\right)$. The integral representation (144) yields

$$
\begin{aligned}
\left|W^{d}(\tau, m, \epsilon)\right| & \leq k^{\prime} \int_{0}^{+\infty} \Phi(1+|m|)^{-\mu} e^{-\beta|m|} \exp \left(\frac{k_{1}}{2} \frac{\log ^{2}(r+\delta)}{\log (q)}+\alpha \log (r+\delta)\right) \times \exp \left(-\frac{r^{k^{\prime}}}{|\tau|^{k^{\prime}}} \cos \left(k^{\prime}(\gamma-\arg (\tau))\right)\right) \mathrm{d} r \\
& \leq k^{\prime} \int_{0}^{+\infty} \Phi(1+|m|)^{-\mu} e^{-\beta|m|} \times \exp \left(\frac{k_{1}}{2} \frac{\log ^{2}(r+\delta)}{\log (q)}+\alpha \log (r+\delta)\right) \exp \left(-\frac{r^{k^{\prime}}}{|\tau|^{k^{\prime}}} \delta_{2}\right) \mathrm{d} r,
\end{aligned}
$$

whenever $\tau \in S_{d}$ for a well-chosen direction $\gamma$ (that may depend on $\tau$ ) such that $\cos \left(k^{\prime}(\gamma-\arg (\tau))\right) \geq \delta_{2}$ for some fixed constant $0<\delta_{2}<1$ that exists under the requirement (145).

In the second part of the proof, we are scaled down to provide bounds for the next associated function:

$$
\mathbb{E}(x)=\int_{0}^{+\infty} \exp \left(\frac{k_{1}}{2} \frac{\log ^{2}(r+\delta)}{\log (q)}+\alpha \log (r+\delta)\right) \exp \left(-\frac{r^{k^{\prime}}}{x}\right) \mathrm{d} r
$$

when $x>0$ is chosen large enough. The next lemma holds.
Lemma 11. One can select two constants $E_{1}>0$ (depending on $k^{\prime}, k_{1}, \delta, q, \alpha$ ) and $E_{2}>1$ (relying in $k_{1}, k^{\prime}, q$ ) such that

$$
\begin{aligned}
\mathbb{E}(x) \leq & E_{1} \exp \left(\frac{k_{1}}{2} \frac{\log ^{2}\left(x^{1 / k^{\prime}}\right)}{\log (q)}+\left(\alpha+E_{2}\right) \log \left(x^{1 / k^{\prime}}\right)\right) \\
& \times \exp \left\{\left(\frac{k_{1} \log \left(x^{1 / k^{\prime}}\right)}{k^{\prime} \log (q)}+\frac{1}{2}\right) \log \left(\frac{k_{1} \log \left(x^{1 / k^{\prime}}\right)}{k^{\prime} \log (q)}+1\right)\right\},
\end{aligned}
$$

for all $x \geq 2 \delta^{k^{\prime}}$.

Proof. We first make the change of variable $\tilde{r}=r^{k^{\prime}} / x$ in the integral above:

$$
\mathbb{E}(x)=\int_{0}^{+\infty} \exp \left(\frac{k_{1}}{2} \frac{\log ^{2}\left((\widetilde{r})^{1 / k^{\prime}} x^{1 / k^{\prime}}+\delta\right)}{\log (q)}+\alpha \log \left((\widetilde{r})^{1 / k^{\prime}} x^{1 / k^{\prime}}+\delta\right)\right) e^{-\widetilde{r}(\widetilde{r})\left(1 / k^{\prime}\right)-1} x^{1 / k^{\prime}} \mathrm{d} \widetilde{r} .
$$

On the other hand, we need the next expansions:

$$
\begin{aligned}
\log ^{2}\left((\widetilde{r})^{1 / k^{\prime}} x^{1 / k^{\prime}}+\delta\right)= & \log ^{2}\left(x^{1 / k^{\prime}}\right)+2 \log \left(x^{1 / k^{\prime}}\right) \log \left((\widetilde{r})^{1 / k^{\prime}}+\frac{\delta}{x^{1 / k^{\prime}}}\right)+\log ^{2}\left((\widetilde{r})^{1 / k^{\prime}}+\frac{\delta}{x^{1 / k^{\prime}}}\right) \\
& \cdot \log \left((\widetilde{r})^{1 / k^{\prime}} x^{1 / k^{\prime}}+\delta\right)=\log \left(x^{1 / k^{\prime}}\right)+\log \left((\widetilde{r})^{1 / k^{\prime}}+\frac{\delta}{x^{1 / k^{\prime}}}\right)
\end{aligned}
$$

We cut the integral expression in two pieces:

$$
\mathbb{E}(x)=\mathbb{E}_{1}(x)+\mathbb{E}_{2}(x),
$$


where

$$
\begin{aligned}
& \mathbb{E}_{1}(x)=\int_{0}^{\left(1-\left(\delta / x^{1 / k^{\prime}}\right)\right)^{k^{\prime}}} \exp \left(\frac{k_{1}}{2} \frac{\log ^{2}\left((\widetilde{r})^{1 / k^{\prime}} x^{1 / k^{\prime}}+\delta\right)}{\log (q)}+\alpha \log \left((\widetilde{r})^{1 / k^{\prime}} x^{1 / k^{\prime}}+\delta\right)\right) e^{-\widetilde{r} \frac{(\widetilde{r})\left(1 / k^{\prime}\right)-1}{k^{\prime}} x^{1 / k^{\prime}} \mathrm{d} \widetilde{r},} \\
& \mathbb{E}_{2}(x)=\int_{\left(1-\left(\delta / x^{1 / k^{\prime}}\right)\right)^{k^{\prime}}}^{+\infty} \exp \left(\frac{k_{1}}{2} \frac{\log ^{2}\left((\widetilde{r})^{1 / k^{\prime}} x^{1 / k^{\prime}}+\delta\right)}{\log (q)}+\alpha \log \left((\widetilde{r})^{1 / k^{\prime}} x^{1 / k^{\prime}}+\delta\right)\right) e^{-\widetilde{r} \frac{(\widetilde{r})\left(1 / k^{\prime}\right)-1}{k^{\prime}} x^{1 / k^{\prime}} \mathrm{d} \widetilde{r},}
\end{aligned}
$$

provided that $x \geq 2 \delta^{k^{\prime}}$.

We control the first piece $\mathbb{E}_{1}(x)$. We observe that $\log \left((\widetilde{r})^{1 / k^{\prime}}+\left(\delta / x^{1 / k^{\prime}}\right)\right) \leq 0$ when $\widetilde{r} \in\left[0,\left(1-\left(\delta / x^{1 / k^{\prime}}\right)\right)^{k^{\prime}}\right]$. From (154), we deduce the inequalities

$$
\begin{aligned}
& \log ^{2}\left((\widetilde{r})^{1 / k^{\prime}} x^{1 / k^{\prime}}+\delta\right) \leq \log ^{2}\left(x^{1 / k^{\prime}}\right)+\log ^{2}\left((\widetilde{r})^{1 / k^{\prime}}+\frac{\delta}{x^{1 / k^{\prime}}}\right), \\
& \log \left((\widetilde{r})^{1 / k^{\prime}} x^{1 / k^{\prime}}+\delta\right) \leq \log \left(x^{1 / k^{\prime}}\right),
\end{aligned}
$$

for all $\tilde{r} \in\left[0,\left(1-\left(\delta / x^{1 / k^{\prime}}\right)\right)^{k^{\prime}}\right]$ and $x \geq 2 \delta^{k^{\prime}}$. Therefore,

$$
\mathbb{E}_{1}(x) \leq \exp \left(\frac{k_{1}}{2} \frac{\log ^{2}\left(x^{1 / k^{\prime}}\right)}{\log (q)}+\alpha \log \left(x^{1 / k^{\prime}}\right)\right) x^{1 / k^{\prime}} \mathbb{E}_{1.2}(x),
$$

where

$$
\begin{aligned}
\mathbb{E}_{1.2}(x)= & \frac{1}{k^{\prime}} \int_{0}^{\left(1-\left(\delta / x^{1 / k^{\prime}}\right)\right)^{k^{\prime}}} \exp \left(\frac{k_{1}}{2} \frac{\log ^{2}\left((\widetilde{r})^{1 / k^{\prime}}+\left(\delta / x^{1 / k^{\prime}}\right)\right)}{\log (q)}\right) \\
& \cdot e^{-\widetilde{r}}(\widetilde{r})^{\left(1 / k^{\prime}\right)-1} \mathrm{~d} \widetilde{r} .
\end{aligned}
$$

By construction, a constant $E_{1.2}>0$ (depending on $\left.k^{\prime}, k_{1}, \delta, q\right)$ can be found with

$$
\mathbb{E}_{1.2}(x) \leq E_{1.2},
$$

for all $x \geq 2 \delta^{k^{\prime}}$.

In a second step, we evaluate the part $\mathbb{E}_{2}(x)$. Expansion (154) affords us to write

$$
\mathbb{E}_{2}(x) \leq \exp \left(\frac{k_{1}}{2} \frac{\log ^{2}\left(x^{1 / k^{\prime}}\right)}{\log (q)}+\alpha \log \left(x^{1 / k^{\prime}}\right)\right) x^{1 / k^{\prime}} \mathbb{E}_{2.1}(x),
$$

where

$$
\begin{aligned}
\mathbb{E}_{2.1}(x)= & \int_{\left(1-\left(\delta / x^{1 / k^{\prime}}\right)\right)^{k^{\prime}}}^{+\infty}\left((\widetilde{r})^{1 / k^{\prime}}+\frac{\delta}{x^{1 / k^{\prime}}}\right)^{k_{1} \log \left(x^{1 / k^{\prime}}\right) / \log (q)} \\
& \times \exp \left(\frac{k_{1}}{2} \frac{\log ^{2}\left((\widetilde{r})^{1 / k^{\prime}}+\left(\delta / x^{1 / k^{\prime}}\right)\right)}{\log (q)}\right. \\
& \left.+\alpha \log \left((\widetilde{r})^{1 / k^{\prime}}+\frac{\delta}{x^{1 / k^{\prime}}}\right)\right) e^{-\widetilde{r}} \frac{1}{k^{\prime}}(\widetilde{r})^{\left(1 / k^{\prime}\right)-1} \mathrm{~d} \widetilde{r} .
\end{aligned}
$$

Besides, we can check that there exists a constant $E_{2.1}>0$ (depending on $k^{\prime}, k_{1}, \delta, q, \alpha$ ) such that

$$
\begin{aligned}
& \exp \left(\frac{k_{1}}{2} \frac{\log ^{2}\left((\widetilde{r})^{1 / k^{\prime}}+\left(\delta / x^{1 / k^{\prime}}\right)\right)}{\log (q)}+\alpha \log \left((\widetilde{r})^{1 / k^{\prime}}+\frac{\delta}{x^{1 / k^{\prime}}}\right)\right) \\
& \cdot \frac{1}{k^{\prime}}(\widetilde{r})^{\left(1 / k^{\prime}\right)-1} \leq E_{2.1} e^{\widetilde{r} / 2},
\end{aligned}
$$

provided that $\tilde{r} \geq\left(1-\left(\delta / x^{1 / k^{\prime}}\right)\right)^{k^{\prime}}$, when $x \geq 2 \delta^{k^{\prime}}$. We deduce that

$$
\begin{aligned}
\mathbb{E}_{2.1}(x) \leq & E_{2.1} \int_{\left(1-\left(\delta / x^{1 / k^{\prime}}\right)\right)^{k^{\prime}}}^{+\infty}\left((\widetilde{r})^{1 / k^{\prime}}+\frac{\delta}{x^{1 / k^{\prime}}}\right)^{k_{1} \log \left(x^{1 / k^{\prime}}\right) / \log (q)} \\
& \cdot e^{-\widetilde{r} / 2} \mathrm{~d} \widetilde{r} \leq E_{2.1} \mathbb{E}_{2.2}(x),
\end{aligned}
$$

where

$$
\mathbb{E}_{2.2}(x)=\int_{\left(1-\left(1 / 2^{1 / k^{\prime}}\right)\right)^{k^{\prime}}}^{+\infty}\left((\widetilde{r})^{1 / k^{\prime}}+\frac{1}{2^{1 / k^{\prime}}}\right)^{k_{1} \log \left(x^{1 / k^{\prime}}\right) / \log (q)} e^{-\widetilde{r} / 2} \mathrm{~d} \widetilde{r},
$$

when $x \geq 2 \delta^{k^{\prime}}$. Furthermore, one can sort a constant $E_{2.2}>1$ (depending on $k^{\prime}$ ) such that

$$
(\widetilde{r})^{1 / k^{\prime}}+\frac{1}{2^{1 / k^{\prime}}} \leq E_{2.2}(\widetilde{r})^{1 / k^{\prime}},
$$

for all $\widetilde{r} \geq\left(1-\left(1 / 2^{1 / k^{\prime}}\right)\right)^{k^{\prime}}$. Hence,

$$
\begin{aligned}
\mathbb{E}_{2.2}(x) \leq & \left.E_{2.2}^{k_{1} \log \left(x^{1 / k^{\prime}}\right) / \log (q)} \int_{\left(1-\left(1 / 2^{1 / k^{\prime}}\right)\right)^{k^{\prime}}}^{+\infty}(\widetilde{r})\left(k_{1} / k^{\prime}\right)\left(\log \left(x^{1 / k^{\prime}}\right)\right) / \log (q)\right) \\
& \cdot e^{-\widetilde{r} / 2} \mathrm{~d} \widetilde{r} \leq E_{2.2}^{k_{1} \log \left(x^{1 / k^{\prime}}\right) / \log (q)} \mathbb{E}_{2.3}(x),
\end{aligned}
$$

with

$$
\mathbb{E}_{2.3}(x)=\int_{0}^{+\infty}(\widetilde{r})\left(k_{1} / k^{\prime}\right)\left(\log \left(x^{1 / k^{\prime}}\right) / \log (q)\right) e^{-\widetilde{r} / 2} \mathrm{~d} \widetilde{r},
$$

when $x \geq 2 \delta^{k^{\prime}}$. We perform the linear change of variable $h=$ $\widetilde{r} / 2$ in this latter integral

$$
\begin{aligned}
\mathbb{E}_{2.3}(x) & =2^{\left(k_{1} \log \left(x^{1 / k^{\prime}}\right) / k^{\prime} \log (q)\right)+1} \int_{0}^{+\infty} h^{k_{1} \log \left(x^{1 / k^{\prime}}\right) / k^{\prime} \log (q)} e^{-h} \mathrm{~d} h \\
& =2^{k_{1} \log \left(x^{1 / k^{\prime}}\right) / k^{\prime} \log (q)+1} \Gamma\left(\frac{k_{1} \log \left(x^{1 / k^{\prime}}\right)}{k^{\prime} \log (q)}+1\right),
\end{aligned}
$$


in order to express it in terms of the Gamma function $\Gamma(x)$. Keeping in mind the Stirling formula (48), we get a constant $E_{2.3}>0$ (which depends on $k_{1}, k^{\prime}, q, \delta$ ) such that

$$
\begin{aligned}
\mathbb{E}_{2.3}(x) \leq & E_{2.3}\left(\frac{2}{e}\right) \\
& \cdot \exp \left(\left(\frac{k_{1} \log \left(x^{1 / k^{\prime}}\right)}{k^{\prime} \log (q)}+\frac{1}{2}\right) \log \left(\frac{\left.k_{1} \log \left(x^{1 / k^{\prime}}\right) / k^{\prime} \log (q)\right)+1}{k^{\prime} \log (q)}+1\right)\right) \\
\leq & E_{2.3} \exp \left(\left(\frac{k_{1} \log \left(x^{1 / k^{\prime}}\right)}{k^{\prime} \log (q)}+\frac{1}{2}\right) \log \left(\frac{k_{1} \log \left(x^{1 / k^{\prime}}\right)}{k^{\prime} \log (q)}+1\right)\right),
\end{aligned}
$$

for all $x \geq 2 \delta^{k^{\prime}}$.

Finally, splitting (155) together with the collection of bounds (159)-(162) and (165)-(171) gives rise to the expected bound (152).

This last lemma combined with estimate (150) yield the announced upper bounds (146) and (147). In order to deduce the particular estimate (148) from (146) and (147), we observe that for any given $\chi>0$ (even close to 0 ), we can sort a constant $A_{\chi, k, k^{\prime}, q, E_{3}, \delta}>0$ (which relies on $\chi, k, k^{\prime}, q, E_{3}, \delta$ ) such that

$$
\log \left(\frac{k_{1}}{k^{\prime} \log (q)} \log \left(\frac{z}{E_{3}}\right)+1\right) \leq \chi \log (z+\delta)+A_{\chi, k, k^{\prime}, q, E_{3}, \delta}
$$

for all $z \geq 2^{1 / k^{\prime}} E_{3} \delta$.

In the final part of the proof, the function $W^{d}(\tau, m, \epsilon)$ is shown to fulfill the second main equation (69). In this respect, we tread rearwards the construction discussed in Section 3. Indeed, according to the fact that $w^{d}(u, m, \epsilon)$

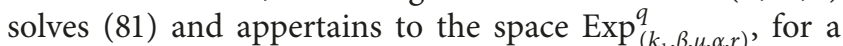
well-chosen sector $U_{d}$, the three identities of Lemma 8 can be applied in order to check that $W^{d}(\tau, m, \epsilon)$ is a genuine solution of the integrodifferential- $q$-difference equation in prepared form (76). Ultimately, a successive play of Lemma 7 followed by Lemma 6 transforms equation (76) into the expected one (69).

\section{Construction of a Finite Set of True Sectorial Solutions to the Main Initial Value Problem}

We return to the first part of the formal constructions undertaken in Section 3 in view of the gain made in solving the two auxiliary problems (81) and (69) throughout Sections 4 and 5 .

We need to state the definition of a good covering in $\mathbb{C}^{*}$, and we introduce a fitted version of a so-called associated sets of sectors to a good covering which is analog to the one proposed in our previous work [3].

Definition 6. Let $\varsigma \geq 2$ be an integer. We consider a set $\underline{\mathscr{E}}$ of open sectors $\mathscr{E}_{p}$ centered at 0 , with radius $\epsilon_{0}>0$ for all $0 \leq p \leq \varsigma-1$ for which the next three properties hold:

(i) The intersection $\mathscr{E}_{p} \cap \mathscr{E}_{p+1}$ is not empty for all $0 \leq p \leq \varsigma-1$ (with the convention that $\mathscr{E}_{\varsigma}=\mathscr{E}_{0}$ ) (ii) The intersection of any three elements of $\underline{\mathscr{E}}$ is empty

(iii) The union $\cup_{p=0}^{\varsigma-1} \mathscr{E}_{p}$ equals $\mathscr{U} \backslash\{0\}$ for some neighborhood $\mathcal{U}$ of 0 in $\mathbb{C}$ $\mathbb{C}^{*}$.

Then, the set of sectors $\underline{\mathscr{E}}$ is named a good covering of

Definition 7. We consider

(i) A good covering $\mathscr{\mathscr { E }}=\left\{\mathscr{E}_{p}\right\}_{0 \leq p \leq c_{-1}}$ of $\mathbb{C}^{*}$ whose radius $\epsilon_{0}$ satisfies $0<\epsilon_{0}<1$

(ii) A set $\underline{U}$ of unbounded sectors $U_{d p}, 0 \leq p \leq \varsigma-1$, centered at 0 with bisecting direction $d_{p} \in \mathbb{R}$ and small opening $\theta_{U_{d_{p}}}>0$

(iii) A set $\underline{S}$ of unbounded sectors $S_{d p}, 0 \leq p \leq \varsigma-1$, centered at 0 with bisecting direction $d_{p} \in \mathbb{R}$ and aperture $0<\theta_{S_{d_{p}}}<\left(\pi / k^{\prime}\right)+\theta_{U_{d_{p}}}$, for some integer
$k^{\prime} \geq 1$

(iv) A fixed bounded sector $\mathscr{T}$ centered at 0 with radius $r_{\mathscr{T}}>0$ and a disc $D(0, r)$ suitably selected in a way that the next features are conjointly satisfied:

(a) Bound (112) holds, provided that $u \in U_{d_{p}} \cup$ $D(0, r)$, for all $0 \leq p \leq \varsigma-1$

(b) The set $\underline{S}$ fulfills the next properties:

(1) The intersection $S_{d_{p}} \cap S_{d_{p+1}}$ is not empty for all $0 \leq p \leq \varsigma-1$ (with the convention that $\left.S_{d_{c}}=S_{d_{0}}\right)$

(2) The union $\cup_{p=0}^{\varsigma-1} S_{d_{p}}$ covers $\mathbb{C} \backslash\{0\}$

(c) For all $0 \leq p \leq \varsigma-1$, all $\epsilon \in \mathscr{E}_{p}$ and all $t \in \mathscr{T}$ :

$$
\epsilon t \in \mathscr{R}_{d_{p}, \Delta} \text {, }
$$

where

$$
\mathscr{R}_{d_{p}, \Delta}=\left\{T \in \frac{\mathbb{C}^{*}}{\left|1+\left(e^{\sqrt{-1} d_{p} / T}\right) r\right|}>\Delta, \quad \text { for all } \mathrm{r} \geq 0\right\},
$$

with $\Delta>0$ any fixed real number close to 0 .

When the above features are verified, we say that the set of data $\{\underline{\mathscr{E}}, \underline{U}, \underline{S}, \mathscr{T}, D(0, r)\}$ is admissible.

We settle now the first principal result of the work. We construct a set of actual holomorphic solutions to the main initial value problem (33) defined on sectors $\mathscr{E}_{p}$, $0 \leq p \leq \varsigma-1$, of a good covering in $\mathbb{C}^{*}$. Besides, we are able to monitor the difference between consecutive solutions on the intersections $\mathscr{E}_{p} \cap \mathscr{E}_{p+1}$.

Theorem 1. We ask the record of requirements (29)-(32), (34), (36), (39), (53), (61), (108), and (116)-(118) to hold. Let us distinguish an admissible set of data

$$
\begin{aligned}
& \underline{\mathscr{A}}=\left\{\underline{\mathscr{E}}=\left\{\mathscr{E}_{p}\right\}_{0 \leq p \leq \varphi^{-1}}, \underline{U}=\left\{U_{d_{p}}\right\}_{0 \leq p \leq c^{-1}},\right. \\
& \left.\underline{S}=\left\{S_{d_{p}}\right\}_{0 \leq p \leq \varsigma-1}, \mathscr{T}, D(0, r)\right\},
\end{aligned}
$$


as described in the definition above.

Then, for a suitable choice of the constants $C_{l}>0$ (c.f. (34)) close enough to 0 for all $l \in I$, a collection $\left\{u_{p}(t, z, \epsilon)\right\}_{0 \leq p \leq c^{-1}}$ of true solutions of (33) can be singled out. More precisely, each function $u_{p}(t, z, \epsilon)$ stands for a bounded holomorphic map on the product $(\mathscr{T} \cap D(0, \sigma)) \times H_{\beta^{\prime}} \times \mathscr{E}_{p}$ for any given $0<\beta^{\prime}<\beta$ and appropriate small radius $\sigma>0$. Additionally, $u_{p}(t, z, \epsilon)$ is represented as a q-Laplace transform of order $k$ and Fourier inverse integral:

$$
\begin{aligned}
u_{p}(t, z, \epsilon)= & \frac{1}{\pi_{q^{1 / k}}(2 \pi)^{1 / 2}} \int_{-\infty}^{+\infty} \int_{L_{\gamma_{p}}} W^{d_{p}}(u, m, \epsilon) \\
& \cdot \frac{1}{\Theta_{q^{1 / k}}(u / \epsilon t)} e^{i z m} \frac{\mathrm{d} u}{u} \mathrm{~d} m,
\end{aligned}
$$

where $L_{\gamma_{p}}=\mathbb{R}_{+} e^{\sqrt{-1} \gamma_{p}} \subset S_{d_{p}} \cup\{0\}$. Furthermore, the map $(\tau, m, \epsilon) \stackrel{{ }_{p}}{\longrightarrow} W^{d_{p}}(\tau, m, \epsilon)$ is itself fashioned as a Laplace transform of order $k^{\prime}$ :

$$
W^{d_{p}}(\tau, m, \epsilon)=k^{\prime} \int_{L_{\gamma_{p}^{\prime}}} w^{d_{p}}(u, m, \epsilon) \exp \left(-\left(\frac{u}{\tau}\right)^{k^{\prime}}\right) \frac{\mathrm{d} u}{u},
$$

whose integration halfline $L_{\gamma_{p}}$ is enclosed in $U_{d_{p}} \cup\{0\}$ and where $(u, m) \longmapsto w^{d_{p}}(u, m, \epsilon)$ belongs to the Banach space $\operatorname{Exp}_{\left(k_{1}, \beta, \mu, \alpha, r\right)}^{q}$ for the unbounded sector $U_{d_{p}}$, provided that $\epsilon \in D\left(0, \epsilon_{0}\right)$.

Finally, some constants $A_{p}, B_{p}>0$ can be found with

$$
\begin{aligned}
& \sup _{t \in \mathscr{T} \cap D(0, \sigma), z \in H_{\beta^{\prime}}}\left|u_{p+1}(t, z, \epsilon)-u_{p}(t, z, \epsilon)\right| \\
& \leq A_{p}\left(B_{p}\right)^{N} \Gamma\left(\frac{N+1}{k^{\prime}}\right) q^{N^{2} / 2 k}|\epsilon|^{N},
\end{aligned}
$$

for all integers $N \geq 1$, all $0 \leq p \leq \varsigma-1$, whenever $\epsilon \in \mathscr{E}_{p+1} \cap \mathscr{E}_{p}$, where by convention, we set $u_{\varsigma}(t, z, \epsilon)=u_{0}(t, z, \epsilon)$.

Proof. We first single out an admissible set of data $\mathscr{A}$. Under the requirements enounced in Theorem 1, Proposition 5 can be called in order to find a family of functions:

$$
W^{d_{p}}(\tau, m, \epsilon)=k^{\prime} \int_{L_{\gamma_{p}^{\prime}}} w^{d_{p}}(u, m, \epsilon) \exp \left(-\left(\frac{u}{\tau}\right)^{k^{\prime}}\right) \frac{\mathrm{d} u}{u},
$$

expressed as a Laplace transform of order $k^{\prime}$ in direction $\gamma_{p}^{\prime}$ such that $L_{\gamma_{p}^{\prime}}=\mathbb{R}_{+} e^{\sqrt{-1} \gamma_{p}^{\prime}} \subset U_{d_{p}} \cup\{0\}$ of a Borel map $w^{d_{p}}(\mathcal{u}$, $m, \epsilon)$ which is holomorphic w.r.t $u$ on $U_{d_{p}} \cup D(0, r)$, w.r.t $\epsilon$ on $D\left(0, \epsilon_{0}\right)$, and continuous relatively to $m \in \mathbb{R}$, coming along with a constant $\Phi^{d p}>0$ such that

$$
\begin{aligned}
\left|w^{d_{p}}(u, m, \epsilon)\right| \leq & \Phi^{d_{p}}(1+|m|)^{-\mu} e^{-\beta|m|} \\
& \cdot|u| \exp \left(\frac{k_{1}}{2} \frac{\log ^{2}(|u|+\delta)}{\log (q)}+\alpha \log (|u|+\delta)\right),
\end{aligned}
$$

for all $u \in U_{d_{p}} \cup D(0, r), m \in \mathbb{R}$, and $\epsilon \in D\left(0, \epsilon_{0}\right)$. Furthermore, the function $W^{d_{p}}(\tau, m, \epsilon)$ solves the first auxiliary integrodifferential $q$-difference equation (69) on $S_{d_{p}} \times \mathbb{R} \times$ $D\left(0, \epsilon_{0}\right)$ and suffers the bounds:

$$
\begin{aligned}
\left|W^{d_{p}}(\tau, m, \epsilon)\right| \leq & \rho_{1}^{d_{p}}(1+|m|)^{-\mu} e^{-\beta|m|} \exp \left(\frac{k_{2}}{2} \frac{\log ^{2}(|\tau|+\delta)}{\log (q)}\right. \\
& \left.+\left(\alpha+\rho_{2}^{d_{p}}\right) \log (|\tau|+\delta)\right),
\end{aligned}
$$

for some constants $\rho_{j}^{d_{p}}>0$, for $j=1,2$, provided that $\tau \in S_{d_{p}}, m \in \mathbb{R}$, and $\epsilon \in D\left(0, \epsilon_{0}\right)$.

We now revisit the first stage of the formal construction from Section 3. Namely, we set the next $q$-Laplace transform of order $k$ and Fourier inverse map

$$
\begin{aligned}
U_{\gamma_{p}}(T, z, \epsilon)= & \frac{1}{\pi_{q^{1 / k}}(2 \pi)^{1 / 2}} \int_{-\infty}^{+\infty} \int_{L_{\gamma_{p}}} W^{d_{p}}(\tau, m, \epsilon) \frac{1}{\Theta_{q^{1 / k}}(\tau / T)} \\
& \cdot e^{i z m} \frac{\mathrm{d} \tau}{\tau} \mathrm{d} m,
\end{aligned}
$$

along a halfline $L_{\gamma_{p}}=\mathbb{R}_{+} e^{\sqrt{-1}} \gamma_{p} \subset S_{d_{p}} \cup\{0\}$. Paying heed to the upper bound (181) and to Lemma 2 together with basic features about Fourier transforms discussed in Definition 4, we notice that $U_{\gamma_{p}}(T, z, \epsilon)$ stands for

(a) A bounded holomorphic function w.r.t $T$ on a domain $\mathscr{R}_{d_{p}, \Delta} \cap D\left(0, r_{0}\right)$ for some small radius $r_{0}>0$, where $\mathscr{R}_{d_{p}, \Delta}$ is described in (173)

(b) A bounded holomorphic application relatively to the couple $(z, \epsilon)$ on $H_{\beta^{\prime}} \times D\left(0, \epsilon_{0}\right)$, for any given $0<\beta^{\prime}<\beta$

Additionally, since $W^{d_{p}}(\tau, m, \epsilon)$ solves (69), Lemma 5 leads to the claim that $U_{\gamma_{p}}(T, z, \epsilon)$ must fulfill the singular equation (64) on $\left(\mathscr{R}_{d_{p}, \Delta} \cap D\left(0, r_{0}\right)\right) \times H_{\beta^{\prime}} \times D\left(0, \epsilon_{0}\right)$.

In conclusion, the function defined as

$$
u_{p}(t, z, \epsilon)=U_{\gamma_{p}}(\epsilon t, z, \epsilon),
$$

represents a bounded holomorphic function w.r.t $t$ on $\mathscr{T} \cap D(0, \sigma)$ for some $\sigma>0$ close enough to $0, \epsilon \in \mathscr{E}_{p}, z \in H_{\beta^{\prime}}$ for any given $0<\beta^{\prime}<\beta$, owing to assumption 3 of Definition 7. Moreover, $u_{p}(t, z, \epsilon)$ solves the main initial value problem (33) on the domain $(\mathscr{T} \cap D(0, \sigma)) \times H_{\beta^{\prime}} \times \mathscr{E}_{p}$, for any $0 \leq p \leq \varsigma-1$.

In the second half of the proof, we explain bound (178). Here, we follow a similar roadmap based on path deformation arguments as in our previous work [3]. Indeed, for $l=p, p+1$, the partial function

$$
\tau \longmapsto \frac{W^{d_{p}}(\tau, m, \epsilon)}{\Theta_{q^{1 / k}}(\tau / \epsilon t) \tau},
$$

is holomorphic on the sector $S_{d}$. By the Cauchy theorem, we can bend each straight halfline $L_{\gamma_{l}}, l=p, p+1$ into the union of three curves with appropriate orientation depicted as follows: 
(1) A halfline $L_{\gamma_{l}, r_{1}}=\left[r_{1},+\infty\right)$ for a given real number $r_{1}>0$

(2) An arc of circle with radius $r_{1}$ denoted $C_{r_{1}, \gamma_{l}, \gamma_{p, p+1}}$ joining the point $r_{1} \exp \left(\sqrt{-1} \gamma_{p, p+1}\right)$ which is taken inside the intersection $S_{d_{p}} \cap S_{d_{p+1}}$ (that is assumed to be nonempty, see Definition $7,2.1$ ) to the halfline $L_{\gamma_{l}, r_{1}}$

(3) A segment $L_{\gamma_{p, p+1}, 0, r_{1}}=\left[0, r_{1}\right] \exp \left(\sqrt{-1} \gamma_{p, p+1}\right)$

As a result, the difference $u_{p+1}-u_{p}$ can be decomposed into a sum of five integrals along these curves:

$$
\begin{aligned}
u_{p+1}(t, z, \epsilon)-u_{p}(t, z, \epsilon)= & \frac{1}{\pi_{q^{1 / k}}(2 \pi)^{1 / 2}} \int_{-\infty}^{+\infty} \int_{L_{\gamma_{p+1}, r_{1}}} W^{d_{p+1}}(\tau, m, \epsilon) \frac{1}{\Theta_{q^{1 / k}}(\tau / \epsilon t)} e^{i z m} \frac{\mathrm{d} \tau}{\tau} \mathrm{d} m \\
& -\frac{1}{\pi_{q^{1 / k}}(2 \pi)^{1 / 2}} \int_{-\infty}^{+\infty} \int_{L_{\gamma_{p}}, r_{1}} W^{d_{p}}(\tau, m, \epsilon) \frac{1}{\Theta_{q^{1 / k}}(\tau / \epsilon t)} e^{i z m} \frac{\mathrm{d} \tau}{\tau} \mathrm{d} m \\
& +\frac{1}{\pi_{q^{1 / k}}(2 \pi)^{1 / 2}} \int_{-\infty}^{+\infty} \int_{C_{r_{1}, \gamma_{p+1}, \gamma_{p}, p+1}} W^{d_{p+1}}(\tau, m, \epsilon) \frac{1}{\Theta_{q^{1 / k}}(\tau / \epsilon t)} e^{i z m \frac{\mathrm{d} \tau}{\tau} \mathrm{d} m} \\
& -\frac{1}{\pi_{q^{1 / k}}(2 \pi)^{1 / 2}} \int_{-\infty}^{+\infty} \int_{C_{r_{1}, \gamma_{p}, \gamma_{p, p+1}}} W^{d_{p}}(\tau, m, \epsilon) \frac{1}{\Theta_{q^{1 / k}}(\tau / \epsilon t)} e^{i z m} \frac{\mathrm{d} \tau}{\tau} \mathrm{d} m \\
& +\frac{1}{\pi_{q^{1 / k}}(2 \pi)^{1 / 2}} \int_{-\infty}^{+\infty} \int_{L_{\gamma_{p, p+1}, 0, r_{1}}}\left(W^{d_{p+1}}(\tau, m, \epsilon)-W^{d_{p}}(\tau, m, \epsilon)\right) \frac{1}{\Theta_{q^{1 / k}}(\tau / \epsilon t)} e^{i z m} \frac{\mathrm{d} \tau}{\tau} \mathrm{d} m .
\end{aligned}
$$

Bounds for the first piece,

$I_{1}=\left|\frac{1}{\pi_{q^{1 / k}}(2 \pi)^{1 / 2}} \int_{-\infty}^{+\infty} \int_{L_{\gamma_{p+1}}, r_{1}} W^{d_{p+1}}(\tau, m, \epsilon) \frac{1}{\Theta_{q^{1 / k}}(\tau / \epsilon t)} e^{i z m} \frac{\mathrm{d} \tau}{\tau} \mathrm{d} m\right|$,

are now considered. The arguments followed are proximate to the ones displayed in the proof of Theorem 1 from [5]. Owing to Lemma 1 and bound (181), we obtain

$$
\begin{aligned}
I_{1} \leq & \frac{1}{\pi_{q^{1 / k}}(2 \pi)^{1 / 2}} \int_{-\infty}^{+\infty} \int_{r_{1}}^{+\infty} \rho_{1}^{d_{p+1}}(1+|m|)^{-\mu} e^{-\beta|m|} \\
& \times \exp \left(\frac{k_{2}}{2} \frac{\log ^{2}(r+\delta)}{\log (q)}+\left(\alpha+\rho_{2}^{d_{p+1}}\right) \log (r+\delta)\right) \\
& \cdot \frac{1}{C_{q, k} \Delta\left(r^{1 / 2} /|\epsilon t|^{1 / 2}\right)} \exp \left(-\frac{k}{2} \frac{\log ^{2}(r /|\epsilon t|)}{\log (q)}\right) \\
& \times \exp (-m \operatorname{Im}(z)) \frac{\mathrm{d} r}{r},
\end{aligned}
$$

for all $\epsilon \in \mathscr{E}_{p+1} \cap \mathscr{E}_{p}, t \in \mathscr{T} \cap D(0, \sigma)$, and $z \in H_{\beta^{\prime}}$. We need the next two expansions:

$$
\begin{aligned}
\log ^{2}(r+\delta)= & \log ^{2}(r)+2 \log (r) \log \left(1+\frac{\delta}{r}\right)+\log ^{2}\left(1+\frac{\delta}{r}\right) \\
\log ^{2}\left(\frac{r}{|\epsilon t|}\right)= & \log ^{2}(r)-2 \log (r) \log (|\epsilon|)-2 \log (r) \log (|t|) \\
& +\log ^{2}(|\epsilon|)+2 \log (|\epsilon|) \log (|t|)+\log ^{2}(|t|) .
\end{aligned}
$$

Hence,

$$
\begin{aligned}
I_{1} \leq & \frac{\rho_{1}^{d_{p+1}}}{\pi_{q^{1 / k}}(2 \pi)^{1 / 2} C_{q, k} \Delta}|\epsilon t|^{1 / 2} \exp \left(-\frac{k}{2} \frac{\log ^{2}(|\epsilon|)}{\log (q)}\right) \\
& \times \int_{-\infty}^{+\infty} \exp \left(-\left(\beta-\beta^{\prime}\right)|m|\right) \mathrm{d} m \exp \left(-\frac{k}{\log (q)} \log (|\epsilon|) \log (|t|)\right) \\
& \cdot \exp \left(-\frac{k}{2 \log (q)} \log ^{2}(|t|)\right) \\
& \times \int_{r_{1}}^{+\infty} \exp \left(-\frac{\log ^{2}(r)}{2 \log (q)}\left(k-k_{2}\right)\right) \frac{1}{r^{3 / 2}} \\
& \cdot \exp \left(\frac{k_{2}}{\log (q)} \log (r) \log \left(1+\frac{\delta}{r}\right)+\frac{k_{2}}{2 \log (q)} \log ^{2}\left(1+\frac{\delta}{r}\right)\right. \\
& +\left(\alpha+\rho_{2}^{d_{p+1}}\right) \log (r+\delta)+\frac{k}{\log (q)} \log (r) \log (|t|) \\
& \left.+\frac{k}{\log (q)} \log (r) \log (|\epsilon|)\right) \mathrm{d} r,
\end{aligned}
$$

for all $\epsilon \in \mathscr{E}_{p+1} \cap \mathscr{E}_{p}, t \in \mathscr{T} \cap D(0, \sigma)$, and $z \in H_{\beta^{\prime}}$. We now specify estimates for some pieces of these last upper bounds. Namely, since $\log (1+x) \sim x$ as $x$ tends to 0 , we get a constant $A_{1.1}>0$ (depending on $r_{1}, \delta$ ) such that

$$
\log (r) \log \left(1+\frac{\delta}{r}\right) \leq A_{1.1}
$$

for all $r \geq r_{1}$. Since $0<\epsilon_{0}<1$, we also notice that 


$$
\begin{array}{r}
\exp \left(-\frac{k}{\log (q)} \log (|\epsilon|) \log (|t|)\right) \leq|\epsilon|^{-(k / \log (q)) \log \left(r_{\mathscr{T}}\right)}, \\
\quad \exp \left(\frac{k}{\log (q)} \log (r) \log (|\epsilon|)\right) \leq|\epsilon|^{(k / \log (q)) \log \left(r_{1}\right)},
\end{array}
$$

for all $t \in \mathscr{T} \cap D(0, \sigma), \quad \epsilon \in \mathscr{E}_{p+1} \cap \mathscr{E}_{p}, \quad$ and $\quad r \geq r_{1}$. Furthermore,

$$
\exp \left(\frac{k}{\log (q)} \log (r) \log (|t|)\right) \leq|t|^{(k / \log (q)) \log \left(r_{1}\right)},
$$

whenever $r_{1} \leq r \leq 1$ and $0<\sigma<1$ together with

$$
\exp \left(\frac{k}{\log (q)} \log (r) \log (|t|)\right) \leq r^{(k / \log (q)) \log (\sigma)}
$$

provided that $r \geq 1$. Finally, there exists a constant $K_{k, r_{1}, q}>0$ (depending on $k, r_{1}, q$ ) with

$$
\sup _{x>0} x^{(k / \log (q)) \log \left(r_{1}\right)} \exp \left(-\frac{k}{2 \log (q)} \log ^{2}(x)\right) \leq K_{k, r_{1}, q} \text {. }
$$

Inequality (189) together with the collection of bounds (190)-(194) yield two constants $I_{1.1}>0$ and $I_{1.2} \in \mathbb{R}$ (which rely on $\left.k, q, \Delta, k_{2}, \delta, r_{1}, \rho_{1}{ }^{p+1}, \rho_{2}{ }^{p+1}, \beta, \beta^{\prime}\right)$ such that

$$
I_{1} \leq I_{1.1}|\epsilon|^{I_{1.2}} \exp \left(-\frac{k}{2} \frac{\log ^{2}(|\epsilon|)}{\log (q)}\right)
$$

for all $\epsilon \in \mathscr{E}_{p+1} \cap \mathscr{E}_{p}, t \in \mathscr{T} \cap D(0, \sigma)$, and $z \in H_{\beta^{\prime}}$. We want to express these last bounds in terms of sequences now. The discussion hinges on the next lemma.

Lemma 12. The following inequality

$$
|\epsilon|^{-N}|\epsilon|^{I_{1.2}} \exp \left(-\frac{k}{2} \frac{\log ^{2}(|\epsilon|)}{\log (q)}\right) \leq q^{I_{1.2}^{2} / 2 k}\left(q^{-I_{1.2} / k}\right)^{N} q^{N^{2} / 2 k}
$$

holds for all $\epsilon \in \mathbb{C}^{*}$, all integers $N \geq 1$.

Proof. By performing the change of variable $x=\log (|\epsilon|)$ with the help of the computation already undertaken in Lemma 4 , we obtain

$$
\begin{aligned}
& \exp \left(\left(I_{1.2}-N\right) \log (|\epsilon|)-\frac{k}{2} \frac{\log ^{2}(|\epsilon|)}{\log (q)}\right) \\
& \quad \leq \sup _{x \in \mathbb{R}} \exp \left(\left(I_{1.2}-N\right) x-\frac{k}{2} \frac{x^{2}}{\log (q)}\right) \leq q^{\left(I_{1.2}-N\right)^{2} / 2 k} \\
& =q^{I_{1.2}^{2} / 2 k}\left(q^{-I_{1.2} / k}\right)^{N} q^{N^{2} / 2 k},
\end{aligned}
$$

for all given $\epsilon \in \mathbb{C}^{*}$ and integer $N \geq 1$.

Consequently to (195) and (196), two constants $I_{1.3}, I_{1.4}>0$ (depending on $I_{1.1}, I_{1.2}, q, k$ ) can be picked up with

$$
I_{1} \leq I_{1.3}\left(I_{1.4}\right)^{N} q^{N^{2} / 2 k}|\epsilon|^{N}
$$

whenever $\epsilon \in \mathscr{E}_{p+1} \cap \mathscr{E}_{p}, t \in \mathscr{T} \cap D(0, \sigma)$, and $z \in H_{\beta^{\prime}}$, for all integers $N \geq 1$.

With a similar discussion, we can exhibit comparable bounds for the next term:

$$
I_{2}=\left|\frac{1}{\pi_{q^{1 / k}}(2 \pi)^{1 / 2}} \int_{-\infty}^{+\infty} \int_{L_{\gamma_{p}}, r_{1}} W^{d_{p}}(\tau, m, \epsilon) \frac{1}{\Theta_{q^{1 / k}}(\tau / \epsilon t)} e^{i z m} \frac{\mathrm{d} \tau}{\tau} \mathrm{d} m\right| .
$$

Namely, two constants $I_{2.1}>0$ and $I_{2.2} \in \mathbb{R}$ (depending on $\left.k, q, \Delta, k_{2}, \delta, r_{1}, \rho_{1}^{d_{p}}, \rho_{2}^{d_{p}}, \beta, \beta^{\prime}\right)$ can be found with

$$
I_{2} \leq I_{2.1}|\epsilon|^{I_{2.2}} \exp \left(-\frac{k}{2} \frac{\log ^{2}(|\epsilon|)}{\log (q)}\right),
$$

for all $\epsilon \in \mathscr{E}_{p+1} \cap \mathscr{E}_{p}, t \in \mathscr{T} \cap D(0, \sigma)$, and $z \in H_{\beta^{\prime}}$. Furthermore, we can single out two constants $I_{2.3}, I_{2.4}>0$ (resting on $I_{2.1}, I_{2.2}, q, k$ ) such that

$$
I_{2} \leq I_{2.3}\left(I_{2.4}\right)^{N} q^{N^{2} / 2 k}|\epsilon|^{N},
$$

provided that $\epsilon \in \mathscr{E}_{p+1} \cap \mathscr{E}_{p}, t \in \mathscr{T} \cap D(0, \sigma)$, and $z \in H_{\beta^{\prime}}$, for all integers $N \geq 1$.

In the next step, we turn to the first integral along an arc of circle:

$$
\begin{aligned}
I_{3}= & \mid \frac{1}{\pi_{q^{1 / k}}(2 \pi)^{1 / 2}} \int_{-\infty}^{+\infty} \int_{C_{r_{1}, \gamma_{p+1}, \gamma_{p}, p+1}} W^{d_{p+1}}(\tau, m, \epsilon) \frac{1}{\Theta_{q^{1 / k}}(\tau / \epsilon t)} \\
& \cdot e^{i z m} \frac{\mathrm{d} \tau}{\tau} \mathrm{d} m \mid .
\end{aligned}
$$

Making use of Lemma 1 and (181), gives rise to the inequality

$$
\begin{aligned}
I_{3} \leq & \frac{1}{\pi_{q^{1 / k}}(2 \pi)^{1 / 2}} \int_{-\infty}^{+\infty} \mid \int_{\gamma_{p+1}}^{\gamma_{p, p+1}} \rho_{1}^{d_{p+1}}(1+|m|)^{-\mu} e^{-\beta|m|} \\
& \times \exp \left(\frac{k_{2}}{2} \frac{\log ^{2}\left(r_{1}+\delta\right)}{\log (q)}+\left(\alpha+\rho_{2}^{d_{p+1}}\right) \log \left(r_{1}+\delta\right)\right) \\
& \cdot \frac{1}{C_{q, k} \Delta r_{1}^{1 / 2} /|\epsilon t|^{1 / 2}} \times \exp \left(-\frac{k}{2} \frac{\log ^{2}\left(r_{1} /|\epsilon t|\right)}{\log (q)}\right) \\
& \cdot \exp (-m \operatorname{Im}(z)) \mathrm{d} \theta \mid \mathrm{d} m,
\end{aligned}
$$

for all $\epsilon \in \mathscr{E}_{p+1} \cap \mathscr{E}_{p}, t \in \mathscr{T} \cap D(0, \sigma)$, and $z \in H_{\beta^{\prime}}$. We require once more the expansion:

$$
\begin{aligned}
\log ^{2}\left(\frac{r_{1}}{|\epsilon t|}\right)= & \log ^{2}\left(r_{1}\right)-2 \log \left(r_{1}\right) \log (|\epsilon|) \\
& -2 \log \left(r_{1}\right) \log (|t|)+\log ^{2}(|\epsilon|) \\
& +2 \log (|\epsilon|) \log |t|+\log ^{2}(|t|)
\end{aligned}
$$


We deduce that

$$
\begin{aligned}
I_{3} \leq & \frac{2 \rho_{1}^{d_{p+1}}}{\pi_{q^{1 / k}}(2 \pi)^{1 / 2} C_{q, k} \Delta r_{1}^{1 / 2}\left(\beta-\beta^{\prime}\right)}\left|\gamma_{p+1}-\gamma_{p, p+1}\right| \\
& \times \exp \left(\frac{k_{2}}{2} \frac{\log ^{2}\left(r_{1}+\delta\right)}{\log (q)}+\left(\alpha+\rho_{2}^{d_{p+1}}\right) \log \left(r_{1}+\delta\right)\right) \exp \left(-\frac{k}{2} \frac{\log ^{2}\left(r_{1}\right)}{\log (q)}\right)|\epsilon t|^{1 / 2} \exp \left(-\frac{k}{2} \frac{\log ^{2}(|\epsilon|)}{\log (q)}\right) \\
& \times \exp \left(\frac{k}{\log (q)} \log \left(r_{1}\right) \log (|\epsilon|)-\frac{k}{\log (q)} \log (|\epsilon|) \log (|t|)\right) \exp \left(\frac{k}{\log (q)} \log \left(r_{1}\right) \log (|t|)\right) \\
& \times \exp \left(-\frac{k}{2} \frac{\log ^{2}(|t|)}{\log ^{2}(q)}\right) .
\end{aligned}
$$

Owing to the hypothesis $0<\epsilon_{0}<1$, we check that (191) holds and bearing in mind (194), we arrive at the existence of two constants $I_{3.1}>0$ and $I_{3.2} \in \mathbb{R}$ (depending on the constants $\left.k, q, \Delta, k_{2}, \delta, r_{1}, \rho_{1}^{d_{p+1}}, \rho_{2}^{d_{p+1}}, \beta, \beta^{\prime}\right)$ with

$$
I_{3} \leq I_{3.1}|\epsilon|^{I_{3.2}} \exp \left(-\frac{k}{2} \frac{\log ^{2}(|\epsilon|)}{\log (q)}\right),
$$

for all $\epsilon \in \mathscr{E}_{p+1} \cap \mathscr{E}_{p}, t \in \mathscr{T} \cap D(0, \sigma)$, and $z \in H_{\beta^{\prime}}$. Calling back Lemma 12 gives rise to two additional constants $I_{3.3}, I_{3.4}>0$ (subjected to $I_{3.1}, I_{3.2}, q, k$ ) with

$$
I_{3} \leq I_{3.3}\left(I_{3.4}\right)^{N} q^{N^{2} / 2 k}|\epsilon|^{N},
$$

for all $\epsilon \in \mathscr{E}_{p+1} \cap \mathscr{E}_{p}, t \in \mathscr{T} \cap D(0, \sigma)$, and $z \in H_{\beta^{\prime}}$, for all given integers $N \geq 1$.

The second integral along an arc of circle

$$
\begin{aligned}
I_{4}= & \mid \frac{1}{\pi_{q^{1 / k}}(2 \pi)^{1 / 2}} \int_{-\infty}^{+\infty} \int_{C_{r_{1}, \gamma_{p}, \gamma_{p}, p+1}} W^{d p}(\tau, m, \epsilon) \frac{1}{\Theta_{q^{1 / k}}(\tau / \epsilon t)} \\
& \cdot e^{i z m} \frac{\mathrm{d} \tau}{\tau} \mathrm{d} m \mid
\end{aligned}
$$

can be managed in the same way. Indeed, one can single out two constants $I_{4.1}>0$ and $I_{4.2} \in \mathbb{R}$ (relying on $k, q, \Delta, k_{2}$, $\left.\delta, r_{1}, \rho_{1}^{d}, \rho_{2}^{d p}, \beta, \beta^{\prime}\right)$ such that

$$
I_{4} \leq I_{4.1}|\epsilon|^{I_{4.2}} \exp \left(-\frac{k}{2} \frac{\log ^{2}(|\epsilon|)}{\log (q)}\right),
$$

provided that $\epsilon \in \mathscr{E}_{p+1} \cap \mathscr{E}_{p}, t \in \mathscr{T} \cap D(0, \sigma)$, and $z \in H_{\beta^{\prime}}$. Moreover, we can find two constants $I_{4.3}, I_{4.4}>0$ (that hinge on $\left.I_{4.1}, I_{4.2}, q, k\right)$ such that

$$
I_{4} \leq I_{4.3}\left(I_{4.4}\right)^{N} q^{N^{2} / 2 k}|\epsilon|^{N},
$$

whenever $\epsilon \in \mathscr{E}_{p+1} \cap \mathscr{E}_{p}, t \in \mathscr{T} \cap D(0, \sigma)$, and $z \in H_{\beta^{\prime}}$, for all integers $N \geq 1$.

In the remaining part of the proof, we inspect the last integral along the segment:

$$
\begin{aligned}
I_{5}= & \mid \frac{1}{\pi_{q^{1 / k}}(2 \pi)^{1 / 2}} \int_{-\infty}^{+\infty} \int_{L_{\gamma_{p, p+1}, 0, r_{1}}}\left(W^{d_{p+1}}(\tau, m, \epsilon)-W^{d_{p}}(\tau, m, \epsilon)\right) \\
& \times \frac{1}{\Theta_{q^{1 / k}}(\tau / \epsilon t)} e^{i z m} \frac{\mathrm{d} \tau}{\tau} \mathrm{d} m \mid .
\end{aligned}
$$

We require a lead-in lemma which supplies exponential flatness for the difference $W^{d_{p+1}}-W^{d p}$.

Lemma 13. For each $0 \leq p \leq \varsigma-1$, we can sort two constants $K_{p}^{W}, M_{p}^{W}>0$ such that

$$
\begin{aligned}
& \left|W^{d_{p+1}}(\tau, m, \epsilon)-W^{d_{p}}(\tau, m, \epsilon)\right| \\
& \quad \leq K_{p}^{W} \exp \left(-\frac{M_{p}^{W}}{|\tau|^{k^{\prime}}}\right)(1+|m|)^{-\mu} e^{-\beta|m|},
\end{aligned}
$$

for all $\epsilon \in D\left(0, \epsilon_{0}\right)$, all $m \in \mathbb{R}$ and all $\tau \in S_{d_{p+1}} \cap S_{d_{p}} \cap D\left(0, r_{1}\right)$, whenever it is assumed that

$$
0<r_{1} \leq\left(\frac{\delta_{1}-\delta_{2}}{\Delta_{2}}\right)^{1 / k^{\prime}}
$$

for some fixed $0<\delta_{2}<\delta_{1}$ close enough to 0 and any given positive real number $\Delta_{2}>0$, under the convention that $W^{d_{\varsigma}}=W^{d_{0}}$.

Proof. We first observe that all the maps $u \longmapsto w^{d} p(u, m, \epsilon)$, $0 \leq p \leq \varsigma-1$, are analytic continuations on the sector $U_{d_{p}}$ of a unique holomorphic function that we name $u \longmapsto w(u, m, \epsilon)$ on the disc $D(0, r)$ which suffers the same bound (180). Furthermore, the application $u \longmapsto w(u, m, \epsilon) \exp \left(-(u / \tau)^{k^{\prime}}\right) / u$ is holomorphic on $D(0, r)$ when $\tau \in S_{d_{p+1}} \cap S_{d_{p}}$, and its integral is therefore vanishing along an oriented path described as the union of

(a) A segment linking 0 to $(r / 2) \exp \left(\sqrt{-1} \gamma_{p+1}^{\prime}\right)$

(b) An arc of circle with radius $r / 2$ joining the points $(r / 2) \exp \left(\sqrt{-1} \gamma_{p+1}^{\prime}\right)$ and $(r / 2) \exp \left(\sqrt{-1} \gamma_{p}^{\prime}\right)$

(c) A segment attaching $(r / 2) \exp \left(\sqrt{-1} \gamma_{p}^{\prime}\right)$ and the origin 
As a result, taking heed of the integral representation (179) of $W^{d_{p+1}}$ and $W^{d_{p}}$, we can convert the difference $W^{d_{p+1}}-W^{d_{p}}$ into a sum of three integrals:

$$
\begin{aligned}
& W^{d_{p+1}}(\tau, m, \epsilon)-W^{d p}(\tau, m, \epsilon)=k^{\prime} \int_{L_{\gamma_{p+1}^{\prime}, r / 2}} w^{d_{p+1}}(u, m, \epsilon) \exp \left(-\left(\frac{u}{\tau}\right)^{k^{\prime}}\right) \frac{d u}{u} \\
& -k^{\prime} \int_{L_{\gamma_{p}^{\prime}, r / 2}} w^{d_{p}}(u, m, \epsilon) \exp \left(-\left(\frac{u}{\tau}\right)^{k^{\prime}}\right) \frac{\mathrm{d} u}{u}+k^{\prime} \int_{C_{r / 2, \gamma_{p}^{\prime}, \gamma_{p+1}^{\prime}}} w(u, m, \epsilon) \exp \left(-\left(\frac{u}{\tau}\right)^{k^{\prime}}\right) \frac{\mathrm{d} u}{u}
\end{aligned}
$$

where the integrations paths are two halflines and an arc of circle staying aside from the origin that are depicted as follows:

$$
\begin{aligned}
L_{\gamma_{p+1}^{\prime}, r / 2} & =\left[\frac{r}{2},+\infty\right) \exp \left(\sqrt{-1} \gamma_{p+1}^{\prime}\right), \\
L_{\gamma_{p}^{\prime}, r / 2} & =\left[\frac{r}{2},+\infty\right) \exp \left(\sqrt{-1} \gamma_{p}^{\prime}\right), \\
C_{r / 2, \gamma_{p}^{\prime} \gamma_{p+1}^{\prime}} & =\left\{\frac{r}{2} \exp (\sqrt{-1} \theta): \theta \in\left[\gamma_{p}^{\prime}, \gamma_{p+1}^{\prime}\right]\right\} .
\end{aligned}
$$

We consider the first integral along a halfline in the above splitting:

$$
J_{1}=\left|k^{\prime} \int_{L_{\gamma_{p+1}^{\prime}, r / 2}} w^{d_{p+1}}(u, m, \epsilon) \exp \left(-\left(\frac{u}{\tau}\right)^{k^{\prime}}\right) \frac{\mathrm{d} u}{u}\right| .
$$

The direction $\gamma_{p+1}{ }^{\prime}$ (which might depend on $\tau$ ) is properly chosen in order that

$$
\cos \left(k^{\prime}\left(\gamma_{p+1}^{\prime}-\arg (\tau)\right)\right) \geq \delta_{1}
$$

for all $\tau \in S_{d_{p+1}} \cap S_{d_{p}}$, for some fixed $\delta_{1}>0$. Besides, let $\Delta_{2}>0$ be any given positive real number (even close to 0 ). Then, we can find a constant $B_{1.1}>0$ (depending on $k_{1}, k^{\prime}, \delta$, $\left.q, \alpha, r, \Delta_{2}\right)$ such that

$$
\frac{k_{1}}{2} \frac{\log ^{2}(s+\delta)}{\log (q)}+\alpha \log (s+\delta) \leq \Delta_{2} s^{k^{\prime}}+B_{1.1},
$$

for all $s \geq r / 2$. According to estimate (180), we obtain that

$$
\begin{aligned}
J_{1} & \leq k^{\prime} \int_{r / 2}^{+\infty} \omega^{d_{p+1}}(1+|m|)^{-\mu} e^{-\beta|m|} s \exp \left(\frac{k_{1}}{2} \frac{\log ^{2}(s+\delta)}{\log (q)}+\alpha \log (s+\delta)\right) \times \exp \left(-\frac{\cos \left(k^{\prime}\left(\gamma_{p+1}^{\prime}-\arg (\tau)\right)\right)}{|\tau|^{k^{\prime}}} s^{k^{\prime}}\right) \frac{\mathrm{d} s}{s} \\
& \leq k^{\prime} \omega^{d_{p+1}} e^{B_{1.1}}(1+|m|)^{-\mu} e^{-\beta|m|} \int_{r / 2}^{+\infty} \exp \left(-\frac{s^{k^{\prime}}}{|\tau|^{k^{\prime}}}\left(\delta_{1}-|\tau|^{k^{\prime}} \Delta_{2}\right)\right) \mathrm{d} s \\
& \leq k^{\prime} \omega^{d_{p+1}} e^{B_{1.1}}(1+|m|)^{-\mu} e^{-\beta|m|} \int_{r / 2}^{+\infty} \exp \left(-\frac{s^{k^{\prime}}}{|\tau|^{k^{\prime}}} \delta_{2}\right) \mathrm{d} s \\
& \leq k^{\prime} \omega^{d_{p+1}} e^{B_{1.1}}(1+|m|)^{-\mu} e^{-\beta|m|} \frac{|\tau|^{k^{\prime}}}{\delta_{2}} \frac{1}{k^{\prime}(r / 2)^{k^{\prime}-1}} \int_{r / 2}^{+\infty} \frac{\delta_{2}}{|\tau|^{k^{\prime}}} k^{\prime} s^{k^{\prime}-1} \exp \left(-\frac{s^{k^{\prime}}}{|\tau|^{k^{\prime}}} \delta_{2}\right) \mathrm{d} s \\
& =k^{\prime} \omega^{d_{p+1}} e^{B_{1.1}}(1+|m|)^{-\mu} e^{-\beta|m|} \frac{|\tau|^{k^{\prime}}}{\delta_{2}} \frac{1}{k^{\prime}(r / 2)^{k^{\prime}-1}} \exp \left(-\frac{(r / 2)^{k^{\prime}}}{|\tau|^{k^{\prime}}} \delta_{2}\right)
\end{aligned}
$$

for all $\epsilon \in D\left(0, \epsilon_{0}\right)$, all $m \in \mathbb{R}$, provided that $\tau \in S_{d_{p+1}} \cap S_{d_{p}}$ with

$$
|\tau| \leq\left(\frac{\delta_{1}-\delta_{2}}{\Delta_{2}}\right)^{1 / k^{\prime}}
$$

for a given $0<\delta_{2}<\delta_{1}$.
In a similar manner, we disclose bounds for the next integral over a halfline:

$$
J_{2}=\left|k^{\prime} \int_{L_{\gamma_{p}^{\prime}, r / 2}} w^{d_{p}}(u, m, \epsilon) \exp \left(-\left(\frac{u}{\tau}\right)^{k^{\prime}}\right) \frac{\mathrm{d} u}{u}\right| .
$$


Indeed, the direction $\gamma_{p}^{\prime}$ (that relies on $\tau$ ) is properly chosen in order that

$$
\cos \left(k^{\prime}\left(\gamma_{p}^{\prime} \arg (\tau)\right)\right) \geq \delta_{1},
$$

for all $\tau \in S_{d_{p+1}} \cap S_{d_{p}}$, for some fixed $\delta_{1}>0$. The use of (180) together with a record of bounds ressembling (219) allows

$$
J_{2} \leq k^{\prime} \varpi^{d p} e^{B_{1.1}}(1+|m|)^{-\mu} e^{-\beta|m|} \frac{|\tau|^{k^{\prime}}}{\delta_{2}} \frac{1}{k^{\prime}(r / 2)^{k^{\prime}-1}} \exp \left(-\frac{(r / 2)^{k^{\prime}}}{|\tau|^{k^{\prime}}} \delta_{2}\right)
$$

to hold whenever $\epsilon \in D\left(0, \epsilon_{0}\right), m \in \mathbb{R}$, and $\tau \in S_{d_{p+1}} \cap S_{d_{p}}$ restricted to (220) for given $0<\delta_{2}<\delta_{1}$ and $\Delta_{2}>0$.
In the remaining part of the lemma, we evaluate the third integral along an arc of circle:

$$
J_{3}=\left|k^{\prime} \int_{C_{r / 2, \gamma_{p}^{\prime}, \gamma_{p+1}^{\prime}}} w(u, m, \epsilon) \exp \left(-\left(\frac{u}{\tau}\right)^{k^{\prime}}\right) \frac{\mathrm{d} u}{u}\right| .
$$

The circle $C_{r / 2, \gamma_{p}^{\prime}, \gamma_{p+1}^{\prime}}$ satisfies the lower bounds

$$
\cos \left(k^{\prime}(\theta-\arg (\tau))\right) \geq \delta_{1},
$$

for some fixed $\delta_{1}>0$, for all $\theta \in\left[\gamma_{p}^{\prime}, \gamma_{p+1}^{\prime}\right]$ (if $\gamma_{p}^{\prime}<\gamma_{p+1}^{\prime}$ ) or $\theta \in\left[\gamma_{p+1}^{\prime}, \gamma_{p}^{\prime}\right]$ (if $\gamma_{p+1}^{\prime}<\gamma_{p}^{\prime}$ ) granting that $\tau \in S_{d p+1} \cap S_{d_{p}}$. Again, estimate (180) together with (218) for $s=r / 2$ brings on

$$
\begin{aligned}
& J_{3} \leq k^{\prime} \mid \int_{\gamma_{p}^{\prime}}^{\gamma_{p+1}^{\prime}} \max \left(\omega^{d_{p}}, \omega^{d_{p+1}}\right)(1+|m|)^{-\mu} e^{-\beta|m|} \frac{r}{2} \exp \left(\frac{k_{1}}{2} \frac{\log ^{2}((r / 2)+\delta)}{\log (q)}+\alpha \log \left(\frac{r}{2}+\delta\right)\right) \\
& \times \exp \left(-\frac{\cos \left(k^{\prime}(\theta-\arg (\tau))\right)}{|\tau|^{k^{\prime}}}\left(\frac{r}{2}\right)^{k^{\prime}}\right) \mathrm{d} \theta \mid \\
& \leq k^{\prime} \max \left(\omega^{d_{p}}, \Phi^{d_{p+1}}\right)\left|\gamma_{p+1}^{\prime}-\gamma_{p}^{\prime}\right| e^{B_{1.1}} \times(1+|m|)^{-\mu} e^{-\beta|m|} \frac{r}{2} \\
& \times \exp \left(-\frac{(r / 2)^{k^{\prime}}}{|\tau|^{k^{\prime}}}\left(\delta_{1}-|\tau|^{k^{\prime}} \Delta_{2}\right)\right) \leq k^{\prime} \max \left(\omega^{d}, \omega^{d_{p+1}}\right)\left|\gamma_{p+1}^{\prime}-\gamma_{p}^{\prime}\right| e^{B_{1.1}} \\
& \times(1+|m|)^{-\mu} e^{-\beta|m|} \frac{r}{2} \times \exp \left(-\frac{(r / 2)^{k^{\prime}}}{|\tau|^{k^{\prime}}} \delta_{2}\right)
\end{aligned}
$$

for all $\epsilon \in D\left(0, \epsilon_{0}\right), m \in \mathbb{R}$ and $\tau \in S_{d_{p+1}} \cap S_{d_{p}}$ submitted to (220) for given $0<\delta_{2}<\delta_{1}$ and $\Delta_{2}>0$.

By collecting the above inequalities (219), (223), and (226) applied to the splitting of (214), we achieve the announced bounds (212).

Onwards, we take for granted that the real number $r_{1}>0$ selected in the above deformation (1), (2), and (3) suffers restriction (213) and $0<r_{1} \leq 1$. Bound (212) in a row with Lemma 1 yields:

$$
I_{5} \leq \frac{K_{p}^{W}}{\pi_{q^{1 / k}}(2 \pi)^{1 / 2} C_{q, k} \Delta} \int_{-\infty}^{+\infty} \exp \left(-\left(\beta-\beta^{\prime}\right)|m|\right) \mathrm{d} m|\epsilon t|^{1 / 2} \widetilde{I}_{5}(\epsilon t)
$$

where

$$
\widetilde{I}_{5}(\epsilon t)=\int_{0}^{r_{1}} \exp \left(-\frac{M_{p}^{W}}{r^{k^{\prime}}}\right) \exp \left(-\frac{k}{2} \frac{\log ^{2}(r /|\epsilon t|)}{\log (q)}\right) \frac{1}{r^{3 / 2}} \mathrm{~d} r,
$$

for all $\epsilon \in \mathscr{E}_{p+1} \cap \mathscr{E}_{p}$, all $t \in \mathscr{T} \cap D(0, \sigma)$ and all $z \in H_{\beta^{\prime}}$. Bound control given below in (235) are now provided for this parameter depending on last integral. The ongoing reasoning leans on the next elementary lemma.

\section{Lemma 14}

(1) The next inequality

$$
\left(\frac{1}{r}\right)^{N} \exp \left(-\frac{M_{p}^{W}}{r^{k^{\prime}}}\right) \leq C_{\Gamma, k^{\prime}} C^{N} \frac{N^{1 / 2}}{k^{\prime}} \Gamma \frac{N}{k^{\prime}}
$$

holds for all integers $N \geq 1$ and all positive real numbers $r>0$, where $C=\left(1 / M_{p}^{W}\right)^{1 / k^{\prime}}$ and $C_{\Gamma, k^{\prime}}>0$ is a constant depending on $k^{\prime}$.

(2) For all $r>0$, all $\epsilon, t \in \mathbb{C}^{*}$, the estimate

$$
\left(\frac{r}{|\epsilon t|}\right)^{N} \exp \left(-\frac{k}{2} \frac{\log ^{2}(r /|\epsilon t|)}{\log (q)}\right) \leq q^{N^{2} / 2 k}
$$

arises for all integers $N \geq 1$. 
Proof. For the first item (1), using the change of variable $x=(1 / r)^{k^{\prime}}$ we observe that

$$
\begin{aligned}
\sup _{r>0}\left(\frac{1}{r}\right)^{N} \exp \left(-\left(\frac{1}{r}\right)^{k^{\prime}} M_{p}^{W}\right) & =\sup _{x>0} x^{N / k^{\prime}} \exp \left(-x M_{p}^{W}\right) \\
& =\left(\frac{N}{k^{\prime} M_{p}^{W}}\right)^{N / k^{\prime}} \exp \left(\frac{-N}{k^{\prime}}\right),
\end{aligned}
$$

for all integers $N \geq 1$. On the other hand, from the Stirling formula (48), we get a constant $C_{\Gamma, k^{\prime}}>0$ (depending on $k^{\prime}$ ) such that

$$
e^{-x} x^{x} \leq C_{\Gamma, k^{\prime}} x^{1 / 2} \Gamma(x)
$$

for all $x \geq 1 / k^{\prime}$. Gathering (231) and (232) yields (229).
The second item (2) can be treated in a similar way through the successive changes of variables $y=r /|\epsilon t|$ and $x=\log (y)$ by using the computation already carried out in Lemma 4,

$$
\begin{aligned}
& \sup _{r>0, \epsilon, t \in \mathbb{C}^{*}}\left(\frac{r}{|\epsilon t|}\right)^{N} \exp \left(-\frac{k}{2} \frac{\log ^{2}(r /|\epsilon t|)}{\log (q)}\right) \\
& =\sup _{y>0} y^{N} \exp \left(-\frac{k}{2} \frac{\log ^{2}(y)}{\log (q)}\right) \\
& =\sup _{x \in \mathbb{R}} \exp \left(x N-\frac{k}{2 \log (q)} x^{2}\right)=q^{N^{2} / 2 k},
\end{aligned}
$$

whenever $N \geq 1$.

A consecutive application of (229) and (230) gives rise to the bounds

$$
\begin{aligned}
\widetilde{I}_{5}(\epsilon t) & \leq C_{\Gamma, k^{\prime}} C^{N+1}\left(\frac{N+1}{k^{\prime}}\right)^{1 / 2} \Gamma\left(\frac{N+1}{k^{\prime}}\right) \int_{0}^{r_{1}} r^{N+1} \exp \left(-\frac{k}{2} \frac{\log ^{2}(r /|\epsilon t|)}{\log (q)}\right) \frac{1}{r^{3 / 2}} \mathrm{~d} r \\
& =C_{\Gamma, k^{\prime}} C^{N+1}\left(\frac{N+1}{k^{\prime}}\right)^{1 / 2} \Gamma\left(\frac{N+1}{k^{\prime}}\right)|\epsilon t|^{N+1} \int_{0}^{r_{1}}\left(\frac{r}{|\epsilon t|}\right)^{N+1} \exp \left(-\frac{k}{2} \frac{\log ^{2}(r /|\epsilon t|)}{\log (q)}\right) \frac{1}{r^{3 / 2}} \mathrm{~d} r \\
& \leq C_{\Gamma, k^{\prime}} C^{N+1}\left(\frac{N+1}{k^{\prime}}\right)^{1 / 2} \Gamma\left(\frac{N+1}{k^{\prime}}\right)|\epsilon t|^{N+1} \int_{0}^{r_{1}} r^{-1 / 2} \mathrm{~d} r \frac{1}{|\epsilon t|} q^{N^{2} / 2 k} \\
& \leq 2 \sqrt{r_{1}} C_{\Gamma, k^{\prime}} C^{N+1}(\sigma)^{N}\left(\frac{N+1}{k^{\prime}}\right)^{1 / 2} \Gamma\left(\frac{N+1}{k^{\prime}}\right) q^{N^{2} / 2 k}|\epsilon|^{N}
\end{aligned}
$$

for all $\epsilon \in \mathbb{C}^{*}$, all $t \in D(0, \sigma)$, all integers $N \geq 1$. In other words, we get two constants $C_{I_{5}, 1}, C_{I_{5}, 2}>0$ (which rely on $\left.r_{1}, \sigma, k^{\prime}, M_{p}^{W}\right)$ such that

$$
\widetilde{I}_{5}(\epsilon t) \leq C_{I_{5}, 1}\left(C_{I_{5}, 2}\right)^{N} \Gamma\left(\frac{N+1}{k^{\prime}}\right) q^{N^{2} / 2 k}|\epsilon|^{N},
$$

whenever $\epsilon \in \mathbb{C}^{*}, t \in D(0, \sigma)$, for all integers $N \geq 1$.

Finally, blending (227) and (235) yields two constants $I_{5.1}, I_{5.2}>0$ (which rely on $\epsilon_{0}, \sigma, K_{p}^{W}, q, k, C_{q, k}, \Delta$, $\left.\beta, \beta^{\prime}, C_{I_{5}, 1}, C_{I_{5}, 2}\right)$ with the estimate

$$
I_{5} \leq I_{5.1}\left(I_{5.2}\right)^{N} \Gamma\left(\frac{N+1}{k^{\prime}}\right) q^{N^{2} / 2 k}|\epsilon|^{N},
$$

provided that $\epsilon \in \mathscr{E}_{p+1} \cap \mathscr{E}_{p}, t \in \mathscr{T} \cap D(0, \sigma)$, and $z \in H_{\beta^{\prime}}$, for all integers $N \geq 1$.

Lastly, the collection of estimates (198), (201), (207), (210), and (236) applied to the of splitting (185) induces the next bounds

$$
\begin{aligned}
& \sup _{t \in \mathcal{T} \cap D(0, \sigma), z \in H_{\beta^{\prime}}}\left|u_{p+1}(t, z, \epsilon)-u_{p}(t, z, \epsilon)\right| \\
& \leq\left(\sum_{d=1}^{4} I_{d .3}\left(I_{d .4}\right)^{N} q^{N^{2} / 2 k}+I_{5.1}\left(I_{5.2}\right)^{N} \Gamma\left(\frac{N+1}{k^{\prime}}\right) q^{N^{2} / 2 k}\right)|\epsilon|^{N},
\end{aligned}
$$

for all $\epsilon \in \mathscr{E}_{p+1} \cap \mathscr{E}_{p}$, all integers $N \geq 1$. Since the sequence $\left(\Gamma\left(N+1 / k^{\prime}\right)\right)_{N \geq 1}$ grows faster than any geometric sequence $\left(I^{N}\right)_{N \geq 1}$ with ratio $I>0$, the last inequality (237) warrants the forecast bounds (178).

In the next proposition, we show that difference (178) of neighboring solutions of (33) turn out to be flat functions for which accurate bounds are displayed.

Proposition 6. Let $\left\{u_{p}(t, z, \epsilon)\right\}_{0 \leq p \leq \varsigma^{-1}}$ be the set of actual solutions of (33) built up in Theorem 1. Then, we can find constants $\vartheta>0$ and $\widetilde{A}_{p}, \widetilde{B}_{p}>0$ (which rely on $\left.A_{p}, B_{p}, k, k^{\prime}, q\right)$ such that

$$
\begin{aligned}
& \sup _{t \in \mathscr{T} \cap D(0, \sigma), z \in H_{\beta^{\prime}}}\left|u_{p+1}(t, z, \epsilon)-u_{p}(t, z, \epsilon)\right| \\
& \leq \widetilde{A}_{p} \exp \left(-\frac{\log (q)}{2 k} \Psi^{2}(|\epsilon|)\right),
\end{aligned}
$$

where

$$
\Psi(x)=\frac{k}{k^{\prime} \log (q)}\left(\log \left(\frac{\widetilde{B}_{p}}{x^{k^{\prime}}}\right)-\log \left(\log \left(\frac{\widetilde{B}_{p}}{x^{k^{\prime}}}\right)\right)\right)-1,
$$


provided that $\epsilon \in \mathscr{E}_{p+1} \cap \mathscr{E}_{p}$ with $|\epsilon|<\vartheta$, for all $0 \leq p \leq \varsigma-1$, where by convention $u_{\varsigma}=u_{0}$.

Proof. Let $A_{p}, B_{p}>0$ be real numbers and $k, k^{\prime} \geq 1$ be integers. We define the function

$$
f(x)=\inf _{N \geq 1} A_{p} B_{p}^{N} \Gamma\left(\frac{N+1}{k^{\prime}}\right) q^{N^{2} / 2 k} x^{N},
$$

for all $x>0$. Keeping in mind the Stirling formula (48), we can find two constants $C, D>0$ (depending on $k^{\prime}$ ) with

$$
\Gamma\left(\frac{N+1}{k^{\prime}}\right) \leq C D^{N} \exp \left(\frac{N}{k^{\prime}} \log \left(\frac{N}{k^{\prime}}\right)\right),
$$

for all integers $N \geq 1$. Hence,

$$
f(x) \leq h(x)=\inf _{N \geq 1} A_{p, 1} B_{p, 1}^{N}\left(\frac{N}{k^{\prime}}\right)^{N / k^{\prime}} q^{N^{2} / 2 k} x^{N},
$$

where $A_{p, 1}=A_{p} C$ and $B_{p, 1}=B_{p} D$. In the next part of the proof, we exhibit explicit bounds for the function $h(x)$. We follow a similar strategy as in the recent work [7]. We select a real number $\vartheta_{1}>0$ small enough in order that for all given $x \in\left(0, \vartheta_{1}\right)$, there exists a positive real number $\tilde{N}>1$ such that

$$
\frac{\tilde{N}}{k^{\prime}} q^{\tilde{N} k^{\prime} / k}=\frac{1}{B_{p, 1}^{k^{\prime}} x^{k^{\prime}}}
$$

We focus on the integer $N=\tilde{N}$, where $x$ denotes the floor function. By construction, we have $N \leq \widetilde{N}$. Therefore,

$$
\frac{N}{k^{\prime}} q^{\tilde{N} k^{\prime} / k} \leq \frac{\tilde{N}}{k^{\prime}} q^{\tilde{N} k^{\prime} / k}=\frac{1}{B_{p, 1}^{k^{\prime}} x^{k^{\prime}}}
$$

which implies that

$$
B_{p, 1}^{N} x^{N} \leq \frac{1}{\left(N / k^{\prime}\right)^{N / k^{\prime}} q^{N^{2} / k}}
$$

and hence

$$
A_{p, 1} B_{p, 1}^{N}\left(\frac{N}{k^{\prime}}\right)^{N / k^{\prime}} q^{N^{2} / 2 k} x^{N} \leq A_{p, 1} q^{-N^{2} / 2 k} .
$$

On the other hand, we can express $\tilde{N}$ in term of the variable $x$ by means of the Lambert function. Namely, we set $W(z)$ as the principal branch of the Lambert function defined on $\left(-e^{-1},+\infty\right)$ and which solves the functional equation

$$
W(z) \exp (W(z))=z,
$$

for all $z \in\left(-e^{-1},+\infty\right)$. Since relation (243) can be recast in the form

$$
\left(\frac{\tilde{N} k^{\prime}}{k} \log (q)\right) \exp \left(\frac{\tilde{N} k^{\prime}}{k} \log (q)\right)=\frac{\left(k^{\prime}\right)^{2} \log (q)}{k B_{p, 1}^{k^{\prime}} x^{k^{\prime}}},
$$

we deduce that

$$
\tilde{N}=\frac{k}{k^{\prime} \log (q)} W\left(\frac{\left(k^{\prime}\right)^{2} \log (q)}{k B_{p, 1}^{k^{\prime}} x^{k^{\prime}}}\right)
$$

Furthermore, owing to the paper [23], the next sharp lower bounds

$$
W(z) \geq \log (z)-\log (\log (z)),
$$

hold for all $z>e$. Finally, since $\tilde{N}<N+1$, the above facts (246)-(250) give rise to the bounds

$$
A_{p, 1} B_{p, 1}^{N}\left(\frac{N}{k^{\prime}}\right)^{N / k^{\prime}} q^{N^{2} / 2 k} x^{N} \leq A_{p, 1} \exp \left(-\frac{\log (q)}{2 k} \Psi^{2}(x)\right),
$$

where

$$
\begin{aligned}
\Psi(x)= & \frac{k}{k^{\prime} \log (q)}\left(\log \left(\frac{\left(k^{\prime}\right)^{2} \log (q)}{k B_{p, 1}^{k^{\prime}} x^{k^{\prime}}}\right)\right. \\
& \left.-\log \left(\log \left(\frac{\left(k^{\prime}\right)^{2} \log (q)}{k B_{p, 1}^{k^{\prime}} x^{k^{\prime}}}\right)\right)\right)-1,
\end{aligned}
$$

provided that $0<x<\vartheta=\min \left(\vartheta_{1}, \vartheta_{2}\right)$, where $\vartheta_{2}=\left(\left(k^{\prime}\right)^{2} \log (q) / k B_{p, 1}^{k^{\prime}} e\right)^{1 / k^{\prime}}$. Finally, from (242) and (251), we deduce that

$$
f(x) \leq A_{p, 1} \exp \left(-\frac{\log (q)}{2 k} \Psi^{2}(x)\right),
$$

whenever $0<x<\vartheta$, which implies the forseen bounds (238) when looking back to estimate (178).

\section{Asymptotic Expansions in the Perturbation Parameter}

7.1. Asymptotic Expansions with Double Gevrey and q-Gevrey Scales: A Related Version of the Ramis-Sibuya Theorem. We first put forward the notion of asymptotic expansion with double Gevrey and $q$-Gevrey scales for formal power series introduced by Lastraet al. [6]. Here, we need a version that involves Banach valued functions which represents a straightforward adaptation of the original setting.

Definition 8. Let $\left(\mathscr{F},\|.\|_{\mathscr{F}}\right)$ be a complex Banach space. We set $k, k^{\prime} \geq 1$ as two integers and $q>1$ as a real number. Let $\mathscr{E}$ be a bounded sector in $\mathbb{C}^{*}$ centered at 0 and $f: \mathscr{E} \longrightarrow \mathscr{F}$ be a holomorphic function. Then, $f$ is said to possess the formal series

$$
\widehat{f}(\epsilon)=\sum_{n \geq 0} a_{n} \epsilon^{n} \in \mathscr{F}[[\epsilon]],
$$

as Gevrey asymptotic expansion of mixed order $\left(1 / k^{\prime} ;(q, 1 / k)\right)$ on $\mathscr{E}$ if for each closed proper subsector $\mathscr{W}$ of $\mathscr{E}$ centered at 0 , one can choose two constants $C, M>0$ with

$$
\left\|f(\epsilon)-\sum_{n=0}^{n} a_{n} \epsilon^{n}\right\|_{\mathscr{F}} \leq C M^{N+1} \Gamma\left(\frac{N+2}{k^{\prime}}\right) q^{(N+1)^{2} / 2 k}|\epsilon|^{N+1},
$$

for all integers $N \geq 0$ and any $\epsilon \in \mathscr{W}$.

In the literature, the Ramis-Sibuya theorem is known as a cohomological criterion which ensures the existence of a 
common Gevrey asymptotic expansion of a given order for families of sectorial holomorphic functions (see [24], p.121 or [25], Lemma XI-2-6). Here, we propose a variant of this result which is adapted to the Gevrey asymptotic expansions of the mixed order disclosed in the above definition.

Theorem 2. Consider a complex Banach space $\left(\mathscr{F},|| .\left.\right|_{\mid \mathscr{F}}\right)$ and set a good covering $\left\{\mathscr{E}_{p}\right\}_{0 \leq p \leq \varsigma_{-1}}$ in $\mathbb{C}^{*}$ (described in Definition 6). Let $\left\{G_{p}\right\}_{0 \leq p \leq \varsigma-1}$ be a set of holomorphic maps $G_{p}$ from $\mathscr{E}_{p}$ into $\mathscr{F}$. We define the cocycle $\Delta_{p}(\epsilon)=$ $G_{p+1}(\epsilon)-G_{p}(\epsilon), 0 \leq p \leq \varsigma-1$, that stands for a holomorphic function from $Z_{p}=\mathscr{E}_{p+1} \cap \mathscr{E}_{p}$ into $\mathscr{F}$, with the convention $\mathscr{E}_{\varsigma}=\mathscr{E}_{0}$ and $G_{\zeta}=G_{0}$.

Assume that the ensuing two requirements hold.

(1) The functions $G_{p}(\epsilon)$ are bounded on $\mathscr{E}_{p}$, for $0 \leq p \leq \varsigma-1$,

(2) The functions $\Delta_{p}(\epsilon)$ suffer the next sequential constraint on $Z_{p}$; there exist two constants $A_{p}, B_{p}>0$ with

$$
\left\|\Delta_{p}(\epsilon)\right\|_{\mathscr{F}} \leq A_{p}\left(B_{p}\right)^{N} \Gamma\left(\frac{N+1}{k^{\prime}}\right) q^{N^{2} / 2 k}|\epsilon|^{N},
$$

provided that $\epsilon \in Z_{p}$, for all integers $N \geq 1$, all $0 \leq p \leq \varsigma-1$. In other words, $\Delta_{p}(\epsilon)$ has the null formal series $\widehat{0}$ as Gevrey asymptotic expansion of mixed order $\left(1 / k^{\prime} ;(q, 1 / k)\right)$ on $Z_{p}$, for $0 \leq p \leq \varsigma-1$.

Then, all the functions $G_{p}(\epsilon), 0 \leq p \leq \varsigma-1$, share a common formal power series $\widehat{G}(\epsilon) \in \mathscr{F}[[\epsilon]]$ as Gevrey asymptotic expansion of mixed order $\left(1 / k^{\prime} ;(q, 1 / k)\right)$ on $\mathscr{E}_{p}$.

Proof. The entire discussion leans on the following central lemma.

Lemma 15. For all $0 \leq p \leq \varsigma-1$, the cocycle $\Delta_{p}(\epsilon)$ splits, which means that bounded holomorphic functions $\Psi_{p}: \mathscr{E}_{p} \longrightarrow \mathscr{F}$ can be singled out with the next feature:

$$
\Delta_{p}(\epsilon)=\Psi_{p+1}(\epsilon)-\Psi_{p}(\epsilon),
$$

for all $\epsilon \in Z_{p}$, where by convention $\Psi_{\zeta}=\Psi_{0}$. Furthermore, $a$ sequence $\left\{\varphi_{m}\right\}_{m \geq 0}$ of elements in $\mathscr{F}$ can be built up such that for each $0 \leq p \leq \varsigma-1$ and any closed proper subsector $\mathscr{W} \subset \mathscr{E}_{p}$ with apex at 0 , one can find $\widehat{K}_{p}, \widehat{M}_{p}>0$ with

$$
\left\|\Psi_{p}(\epsilon)-\sum_{m=0}^{M} \varphi_{m} \epsilon^{m}\right\|_{\mathscr{F}} \leq \widehat{K}_{p}\left(\widehat{M}_{p}\right)^{M+1} \Gamma\left(\frac{M+2}{k^{\prime}}\right) q^{(M+1)^{2} / 2 k}|\epsilon|^{M+1},
$$

for all $\epsilon \in \mathscr{W}$, all integers $M \geq 0$.

Proof. The proof mimics the arguments of Lemma XI-2-6 from [25] with fitting adjustment in the asymptotic expansions of the functions $\Psi_{p}$ constructed by means of the Cauchy-Heine transform.

For all $0 \leq p \leq \varsigma-1$, we choose a segment:

$$
C_{p}=\left\{t e^{\sqrt{-1} \theta_{p}}, t \in[0, r]\right\} \subset \mathscr{E}_{p} \cap \mathscr{E}_{p+1} .
$$

These $\varsigma$ segments divide the open punctured disc $D(0, r) \backslash\{0\}$ into $\varsigma$ open sectors $\widetilde{\mathscr{E}}_{0}, \ldots, \widetilde{\mathscr{E}}_{\varsigma^{-1}}$, where

$$
\widetilde{\mathscr{E}}_{p}=\left\{\epsilon \in \frac{\mathbb{C}^{*}}{\theta_{p-1}}<\arg (\epsilon)<\theta_{p},|\epsilon|<r\right\}, \quad 0 \leq p \leq \varsigma-1,
$$

where by convention $\theta_{-1}=\theta_{\varsigma-1}$. Let

$$
\Psi_{p}(\epsilon)=\frac{-1}{2 \pi \sqrt{-1}} \sum_{h=0}^{\varsigma-1} \int_{C_{h}} \frac{\Delta_{h}(\xi)}{\xi-\epsilon} \mathrm{d} \xi,
$$

for all $\epsilon \in \widetilde{\mathscr{E}}_{p}$, for $0 \leq p \leq \varsigma-1$, be defined as a sum of Cauchy-Heine transforms of the functions $\Delta_{h}(\epsilon)$. By deformation of the paths $C_{p-1}$ and $C_{p}$ without moving their endpoints and letting the other paths $C_{h}, h \neq p-1, p$ untouched (with the convention that $C_{-1}=C_{\zeta-1}$ ), one can continue analytically the function $\Psi_{p}$ onto $\mathscr{E}_{p}$. Therefore, $\Psi_{p}$ defines a holomorphic function on $\mathscr{E}_{p}$, for all $0 \leq p \leq \varsigma-1$.

Now, take $\epsilon \in \mathscr{E}_{p} \cap \mathscr{E}_{p+1}$. In order to compute $\Psi_{p+1}(\epsilon)-\Psi_{p}(\epsilon)$, we write

$$
\begin{gathered}
\Psi_{p}(\epsilon)=\frac{-1}{2 \pi \sqrt{-1}} \int_{\widehat{C}_{p}} \frac{\Delta_{p}(\xi)}{\xi-\epsilon} \mathrm{d} \xi+\frac{-1}{2 \pi \sqrt{-1}} \sum_{h=0, h \neq p}^{\varsigma^{-1}} \int_{C_{h}} \frac{\Delta_{h}(\xi)}{\xi-\epsilon} \mathrm{d} \xi, \\
\Psi_{p+1}(\epsilon)=\frac{-1}{2 \pi \sqrt{-1}} \int_{\check{C}_{p}} \frac{\Delta_{l}(\xi)}{\xi-\epsilon} \mathrm{d} \xi+\frac{-1}{2 \pi \sqrt{-1}} \sum_{h=0, h \neq p}^{\varsigma-1} \int_{C_{h}} \frac{\Delta_{h}(\xi)}{\xi-\epsilon} \mathrm{d} \xi,
\end{gathered}
$$

where the paths $\widehat{C}_{p}$ and $\check{C}_{p}$ are obtained by deforming the same path $C_{p}$ without moving its endpoints in such a way that

(a) $\widehat{C}_{p} \subset \mathscr{E}_{p} \cap \mathscr{E}_{p+1}$ and $\check{C}_{p} \subset \mathscr{E}_{p} \cap \mathscr{E}_{p+1}$

(b) $\Gamma_{p, p+1}:=-\check{C}_{p}+\widehat{C}_{p}$ is a simple closed curve with positive orientation whose interior contains $\epsilon$

Therefore, due to the residue formula, we can write

$$
\Psi_{p+1}(\epsilon)-\Psi_{p}(\epsilon)=\frac{1}{2 \pi \sqrt{-1}} \int_{\Gamma_{p, p+1}} \frac{\Delta_{p}(\xi)}{\xi-\epsilon} \mathrm{d} \xi=\Delta_{p}(\epsilon),
$$

for all $\epsilon \in \mathscr{E}_{p} \cap \mathscr{E}_{p+1}$, for all $0 \leq p \leq \varsigma-1$ (with the convention that $\Psi_{\zeta}=\Psi_{0}$ ).

In a second step, we derive asymptotic properties of $\Psi_{p}$. We fix an $0 \leq p \leq \varsigma-1$ and a proper closed sector $\mathscr{W}$ contained in $\mathscr{E}_{p}$. Let $\widetilde{C}_{p}$ (resp. $\widetilde{C}_{p-1}$ ) be a path obtained by deforming $C_{p}$ (resp. $C_{p-1}$ ) without moving the endpoints in order that $\mathscr{W}$ is contained in the interior of the simple closed curve $\widetilde{C}_{p-1}+\gamma_{p}-\widetilde{C}_{p}$ (which is itself contained in $\mathscr{E}_{p}$ ), where $\gamma_{p}$ is a circular arc joining the two points $r e^{\sqrt{-1} \theta_{p-1}}$ and $r e^{\sqrt{-1} \theta_{p}}$. We get the representation

$$
\begin{aligned}
\Psi_{p}(\epsilon)= & \frac{-1}{2 \pi \sqrt{-1}} \int_{\widetilde{C}_{p}} \frac{\Delta_{p}(\xi)}{\xi-\epsilon} \mathrm{d} \xi+\frac{-1}{2 \pi \sqrt{-1}} \int_{\widetilde{C}_{p-1}} \frac{\Delta_{p-1}(\xi)}{\xi-\epsilon} \mathrm{d} \xi \\
& +\frac{-1}{2 \pi \sqrt{-1}} \sum_{h=0, h \neq p, p-1}^{\nu-1} \int_{C_{h}} \frac{\Delta_{h}(\xi)}{\xi-\epsilon} \mathrm{d} \xi,
\end{aligned}
$$


for all $\epsilon \in \mathscr{W}$. One assumes that the path $\widetilde{C}_{p}$ is given as the union of a segment $L_{p}=\left\{t e^{\sqrt{-1}} w_{p} / t \in\left[0, r_{1}\right]\right\}$, where $r_{1}<r$ and $w_{p}>\theta_{p}$ and a curve $\Gamma_{p}=\left\{\mu_{p}(\tau) / \tau \in[0,1]\right\}$ such that $\mu_{p}(0)=r_{1} e^{\sqrt{-1} w_{p}}, \mu_{p}(1)=r e^{\sqrt{-1} \theta_{p}}$, and $r_{1} \leq\left|\mu_{p}(\tau)\right|<r$ for all $\tau \in[0,1)$. We also assume that there exists a positive number $\sigma<1$ with $|\epsilon| \leq \sigma r_{1}$ for all $\epsilon \in \mathscr{W}$. By construction of the path $\Gamma_{p}$, we get that the function $\epsilon \longmapsto$ $(1 / 2 \pi \sqrt{-1}) \int_{\Gamma_{p}}\left(\Delta_{p}(\xi) / \xi-\epsilon\right) \mathrm{d} \xi$ defines an analytic function on the open disc $D\left(0, r_{1}\right)$.

It remains to give estimates for the integral $\epsilon \longmapsto(1 / 2 \pi \sqrt{-1}) \int_{\Gamma_{p}}\left(\Delta_{p}(\xi) / \xi-\epsilon\right) \mathrm{d} \xi$. Let $M \geq 0$ be an integer. From the usual geometric series expansion, one can write

$$
\frac{1}{2 \pi \sqrt{-1}} \int_{L_{p}} \frac{\Delta_{p}(\xi)}{\xi-\epsilon} \mathrm{d} \xi=\sum_{m=0}^{M} \alpha_{p, m} \epsilon^{m}+\epsilon^{M+1} E_{p, M+1}(\epsilon),
$$

where

$$
\begin{aligned}
\alpha_{p, m} & =\frac{1}{2 \pi \sqrt{-1}} \int_{L_{p}} \frac{\Delta_{l}(\xi)}{\xi^{m+1}} \mathrm{~d} \xi \\
E_{p, M+1}(\epsilon) & =\frac{1}{2 \pi \sqrt{-1}} \int_{L_{p}} \frac{\Delta_{p}(\xi)}{\xi^{M+1}(\xi-\epsilon)} \mathrm{d} \xi
\end{aligned}
$$

for all $\epsilon \in \mathscr{W}$.

Keeping in mind (256) for the special value $N=m+1$ and (266), we get some constants $A_{p}, B_{p}>0$ such that

$$
\begin{aligned}
\left\|\alpha_{p, m}\right\|_{\mathscr{F}} & \leq \frac{1}{2 \pi} \int_{0}^{r_{1}} A_{p}\left(B_{p}\right)^{m+1} \Gamma\left(\frac{m+2}{k^{\prime}}\right) q^{(m+1)^{2} / 2 k} \frac{\tau^{m+1}}{\tau^{m+1}} \mathrm{~d} \tau \\
& \leq \frac{r_{1} A_{p}}{2 \pi}\left(B_{p}\right)^{m+1} \Gamma\left(\frac{m+2}{k^{\prime}}\right) q^{(m+1)^{2} / 2 k},
\end{aligned}
$$

for all $0 \leq m \leq M$. In particular, we deduce the existence of two constants $\widehat{A}_{p}, \widehat{B}_{p}>0$ (depending on $A_{p}, B_{p}, r_{1}, q, k, k^{\prime}$ ) with

$$
\left\|\alpha_{p, m}\right\|_{\mathscr{F}} \leq \widehat{A}_{p}\left(\widehat{B}_{p}\right)^{m} \Gamma\left(\frac{m+1}{k^{\prime}}\right) q^{m^{2} / 2 k},
$$

for all $0 \leq m \leq M$. Indeed, recall from [24], Appendix B, that for any given real number $a>0, \Gamma(x) x^{a} \sim \Gamma(x+a)$ as $x$ tends to $+\infty$. Hence, a constant $K_{k^{\prime}}>0$ (depending on $k^{\prime}$ ) can be sort with

$$
\Gamma\left(\frac{m+2}{k^{\prime}}\right) \leq K_{k^{\prime}}\left(\frac{m+1}{k^{\prime}}\right)^{1 / k^{\prime}} \Gamma\left(\frac{m+1}{k^{\prime}}\right),
$$

for all $m \geq 0$. Consequently, (268) follows from (267) and (269).

Moreover, one can choose a positive number $\eta>0$ (depending on $\mathscr{W}$ ) such that $|\xi-\epsilon| \geq|\xi| \sin (\eta)$ for all $\xi \in L_{p}$ and all $\epsilon \in \mathscr{W}$. Bringing to mind (256) for the peculiar value $N=M+2$ and (266) give rise to two constants $A_{p}, B_{p}>0$ such that

$$
\begin{aligned}
\left\|E_{p, M+1}(\epsilon)\right\|_{\mathscr{F}} & \leq \frac{1}{2 \pi \sin (\eta)} \int_{0}^{r_{1}} A_{p}\left(B_{p}\right)^{M+2} \Gamma\left(\frac{M+3}{k^{\prime}}\right) q^{(M+2)^{2} / 2 k} \frac{\tau^{M+2}}{\tau^{M+2}} \mathrm{~d} \tau \\
& \leq \frac{r_{1} A_{p}}{2 \pi \sin (\eta)}\left(B_{p}\right)^{M+2} \Gamma\left(\frac{M+3}{k^{\prime}}\right) q^{(M+2)^{2} / 2 k} .
\end{aligned}
$$

For that reason, we can find constants $\check{A}_{p}, \check{B}_{p}>0$ (relying on $\left.A_{p}, B_{p}, r_{1}, q, k, k^{\prime}, \eta\right)$ such that

$$
\left\|E_{p, M+1}(\epsilon)\right\|_{\mathscr{F}} \leq \check{A}_{p}\left(\check{B}_{p}\right)^{M+1} \Gamma\left(\frac{M+2}{k^{\prime}}\right) q^{(M+1)^{2} / 2 k},
$$

for all $\epsilon \in \mathscr{W}$. Namely, from (269) we notice that

$$
\Gamma\left(\frac{M+3}{k^{\prime}}\right) \leq K_{k^{\prime}}\left(\frac{M+2}{k^{\prime}}\right)^{1 / k^{\prime}} \Gamma\left(\frac{M+2}{k^{\prime}}\right) .
$$

Using comparable arguments, one can give estimates of the form (265), (266), (268), and (271) for the other integrals

$$
\begin{gathered}
\frac{-1}{2 \pi \sqrt{-1}} \int_{\widetilde{C}_{p-1}} \frac{\Delta_{p-1}^{2}(\xi)}{\xi-\epsilon} \mathrm{d} \xi, \\
\frac{-1}{2 \pi \sqrt{-1}} \int_{C_{h}} \frac{\Delta_{h}^{2}(\xi)}{\xi-\epsilon} \mathrm{d} \xi,
\end{gathered}
$$

for all $h \neq p, p-1$.

As a consequence, for any $0 \leq p \leq \varsigma-1$, there exist coefficients $\varphi_{p, m} \in \mathscr{F}, m \geq 0$, and two constants $\widehat{K}_{p}, \widehat{M}_{p}>0$ such that

$$
\left\|\Psi_{p}(\epsilon)-\sum_{m=0}^{M} \varphi_{p, m} \epsilon^{m}\right\|_{\mathscr{F}} \leq \widehat{K}_{p}\left(\widehat{M}_{p}\right)^{M+1} \Gamma\left(\frac{M+2}{k^{\prime}}\right) q^{(M+1)^{2} / 2 k}|\epsilon|^{M+1},
$$

for all integers $M \geq 0$, all $\epsilon \in \mathscr{W}$.

Besides, identity (263) and sequential assumption (256) imply in particular that the difference $\Psi_{p+1}(\epsilon)-\Psi_{p}(\epsilon)$ has the null formal series $\hat{0}$ as asymptotic expansion (in the Poincaré sense) on $Z_{p}$. Owing to the unicity of the asymptotic expansions on sectors, we deduce that all the formal series $\sum_{m \geq 0} \varphi_{p, m} \epsilon^{m}, 0 \leq p \leq \varsigma-1$, are equal to some formal series denoted $\widehat{G}(\epsilon)=\sum_{m \geq 0} \varphi_{m} \epsilon^{m} \in \mathbb{E}[[\epsilon]]$. Lemma 15 follows.

We introduce the bounded holomorphic functions

$$
a_{p}(\epsilon)=G_{p}(\epsilon)-\Psi_{p}(\epsilon),
$$

for all $0 \leq p \leq \varsigma-1$, all $\epsilon \in \mathscr{E}_{p}$. By definition, for any $p \in\{0, \ldots, \varsigma-1\}$, we observe that

$$
a_{p+1}(\epsilon)-a_{p}(\epsilon)=G_{p+1}(\epsilon)-G_{p}(\epsilon)-\Delta_{p}(\epsilon)=0,
$$

for all $\epsilon \in Z_{p}$. Therefore, each $a_{p}(\epsilon)$ stands for the restriction on $\mathscr{E}_{p}$ of a global holomorphic function called $a(\epsilon)$ on $D(0, r) \backslash\{0\}$. Since $a(\epsilon)$ remains bounded on $D(0, r) \backslash\{0\}$, the origin turns out to be a removable singularity for $a(\epsilon)$ which, as a result, defines a convergent power series on $D(0, r)$.

Finally, one can recast

$$
G_{p}(\epsilon)=a(\epsilon)+\Psi_{p}(\epsilon)
$$


for all $\epsilon \in \mathscr{E}_{p}$, all $0 \leq p \leq \varsigma-1$. Furthermore, $a(\epsilon)$ represents a convergent power series and $\Psi_{p}(\epsilon)$ has the formal series $\widehat{G}(\epsilon)=\sum_{m \geq 0} \varphi_{m} \epsilon^{m}$ as Gevrey asymptotic expansion of mixed order $\left(1 / k^{\prime} ;(q, 1 / k)\right)$ on $\mathscr{E}_{p}$, for all $0 \leq p \leq \varsigma-1$. The conclusion of Theorem 2 follows.

7.2. Parametric Gevrey Asymptotic Expansions of the Mixed Order for the Actual Solutions of the Main Initial Value Problem. Within this section, we explain the second principal result of our work.

Theorem 3. We set $\mathscr{F}$ as the Banach space of complex valuebounded holomorphic functions on the product $(\mathscr{T} \cap D(0, \sigma)) \times H_{\beta^{\prime}}$ equipped with the supremum norm, where the sector $\mathscr{T}$, radius $\sigma$, and width $\beta^{\prime}$ are settled in Theorem 1.

Then, for all $0 \leq p \leq \varsigma-1$, the bounded holomorphic functions $\epsilon \longmapsto u_{p}(t, z, \epsilon)$ from $\mathscr{E}_{p}$ into $\mathscr{F}$ constructed in Theorem 1 share a common formal power series

$$
\widehat{u}(t, z, \epsilon)=\sum_{m \geq 0} h_{m}(t, z) \epsilon^{m} \in \mathscr{F}[[\epsilon]],
$$

as Gevrey asymptotic expansion of mixed order $\left(1 / k^{\prime} ;(q, 1 / k)\right)$. Videlicet, for all $0 \leq p \leq \varsigma-1$, two constants $C_{p}, M_{p}>0$ can be found with

$$
\begin{aligned}
& \sup _{t \in \mathscr{T} \cap D(0, \sigma), z \in H_{\beta^{\prime}}}\left|u_{p}(t, z, \epsilon)-\sum_{m=0}^{n} h_{m}(t, z) \epsilon^{m}\right| \\
& \leq C_{p} M_{p}^{n+1} \Gamma\left(\frac{n+2}{k^{\prime}}\right) q^{(n+1)^{2} / 2 k}|\epsilon|^{n+1}
\end{aligned}
$$

for all integers $n \geq 1$, provided that $\epsilon \in \mathscr{E}_{p}$.

Proof. We focus on the set of functions $u_{p}(t, z, \epsilon)$, $0 \leq p \leq \varsigma-1$, constructed in Theorem 1 . For all $0 \leq p \leq \varsigma-1$, we set

$$
G_{p}(\epsilon):=(t, z) \longmapsto u_{p}(t, z, \epsilon) .
$$

Each $G_{p}$ defines a bounded holomorphic map from $\mathscr{E}_{p}$ into the Banach space $\mathscr{F}$ described in the statement of Theorem 3. Furthermore, bound (178) implies that the cocycle $\Delta_{p}(\epsilon)=G_{p+1}(\epsilon)-G_{p}(\epsilon)$ fulfills the sequential bound (256) on $Z_{p}=\mathscr{E}_{p+1} \cap \mathscr{E}_{p}$ for any $0 \leq p \leq \varsigma-1$. Then, Theorem 2 can be applied in order to get a formal power series $\widehat{G}(\epsilon) \in \mathscr{F}[[\epsilon]]$ which stands for the Gevrey asymptotic expansion of mixed order $\left(1 / k^{\prime} ;(q, 1 / k)\right)$ of each $G_{p}(\epsilon)$ on $\mathscr{E}_{p}$, for all $0 \leq p \leq \varsigma-1$.

\section{Data Availability}

There are no underlying data related to the submitted manuscript.

\section{Conflicts of Interest}

The author declares that there are no conflicts of interest regarding the publication of this article.

\section{References}

[1] J.-P. Ramis, J. Sauloy, and C. Zhang, "Local analytic classification of q-difference equations," Asterisque- Societe Mathematique de France, vol. 355, p. 151, 2013.

[2] R. Gérard and H. Tahara, Singular Nonlinear Partial Differential Equations: Aspects of Mathematics, Friedrich Vieweg and Sohn, Braunschweig, Germany, 1996.

[3] A. Lastra and S. Malek, "On singularly perturbed linear initial value problems with mixed irregular and Fuchsian time singularities," 2019, https://arxiv.org/abs/1901.05210.

[4] W. Balser, "From divergent power series to analytic functions: theory and application of multisummable power series," in Lecture Notes in Mathematics, vol. 1582, p. 108, SpringerVerlag, Berlin, Germany, 1994.

[5] S. Malek, "On parametric Gevrey asymptotics for a q-analog of some linear initial value problem," Funkcialaj Ekvacioj, vol. 60, no. 1, pp. 21-63, 2017.

[6] A. Lastra, S. Malek, and J. Sanz, "On q- asymptotics for linear q- difference-differential equations with Fuchsian and irregular singularities," Journal of Differential Equations, vol. 252, no. 10, pp. 5185-5216, 2012.

[7] H. Yamazawa, Gevrey and q- Gevrey Asymptotics for Some Linear q-Difference-Differential Equations, Talk at RIMS in Kyoto, RIMS, Kyoto, Japan, 2018.

[8] H. Tahara, "q-analogues of Laplace and Borel transforms by means of q-exponentials," Annales de l'institut Fourier (Grenoble), vol. 67, no. 5, pp. 1865-1903, 2017.

[9] H. Tahara and H. Yamazawa, "q-analogue of summability of formal solutions of some linear q-difference-differential equations," Opuscula Mathematica, vol. 35, no. 5, pp. 713738, 2015.

[10] H. Yamazawa, "Holomorphic and singular solutions of q-difference-differential equations of Briot-Bouquet type," Funkcialaj Ekvacioj, vol. 59, no. 2, pp. 185-197, 2016.

[11] D. Pravica, N. Randriampiry, and M. Spurr, "Applications of an advanced differential equation in the study of wavelets," Applied and Computational Harmonic Analysis, vol. 27, no. 1, pp. 2-11, 2009.

[12] D. Pravica, N. Randriampiry, and M. Spurr, "q-advanced models for tsunami and rogue waves," Abstract and Applied Analysis, vol. 2012, Article ID 414060, 26 pages, 2012.

[13] D. Pravica, N. Randriampiry, and M. Spurr, "Solutions of a class of multiplicatively advanced differential equations," Comptes Rendus Mathematique, vol. 356, no. 7, pp. 776-817, 2018.

[14] A. Lastra and S. Malek, "On parametric Gevrey asymptotics for some nonlinear initial value Cauchy problems," Journal of Differential Equations, vol. 259, no. 10, pp. 5220-5270, 2015.

[15] L. Di Vizio and C. Zhang, "On q-summation and confluence," Annales de l'institut Fourier, vol. 59, no. 1, pp. 347-392, 2009.

[16] T. Dreyfus, A. Lastra, and S. Malek, "Multiple-scale analysis for some linear partial q-difference and differential equations with holomorphic coefficients," Advances in Difference Equations, vol. 2019, no. 1, 2019.

[17] A. Lastra and S. Malek, "On q- Gevrey asymptotics for singularly perturbed q- difference-differential problems with an irregular singularity," Abstract and Applied Analysis, vol. 2012, Article ID 860716, 35 pages, 2012.

[18] A. Lastra and S. Malek, "On parametric multilevel q-Gevrey asymptotics for some linear q-difference-differential equations," Advances in Difference Equations, vol. 2015, no. 1, p. 344, 2015.

[19] H. Tahara and H. Yamazawa, "Multisummability of formal solutions to the Cauchy problem for some linear partial 
differential equations," Journal of Differential Equations, vol. 255, no. 10, pp. 3592-3637, 2013.

[20] A. Lastra and S. Malek, "On parametric Gevrey asymptotics for initial value problems with infinite order irregular singularity and linear fractional transforms," Advances in Difference Equations, vol. 2018, no. 1, p. 386, 2018.

[21] O. Costin and S. Tanveer, "Short time existence and Borel summability in the Navier-Stokes equation in $\mathbb{R}^{3}$." Communications in Partial Differential Equations, vol. 34, no. 7-9, pp. 785-817, 2009.

[22] S. Malek, "On Gevrey asymptotics for some nonlinear integro-differential equations," Journal of Dynamical and Control Systems, vol. 16, no. 3, pp. 377-406, 2010.

[23] A. Hoorfar and M. Hassani, "Inequalities on the Lambert W function and hyperpower function," Journal of Inequalities in Pure and Applied Mathematics (JIPAM), vol. 9, no. 2, p. 51, 2008.

[24] W. Balser, Formal Power Sries and Linear Systems of Meromorphic Ordinary Differential Equations, Universitext, Springer-Verlag, New York, USA, 2000.

[25] P. Hsieh and Y. Sibuya, Basic Theory of Ordinary Differential Equations, Universitext, Springer-Verlag, New York, USA, 1999. 


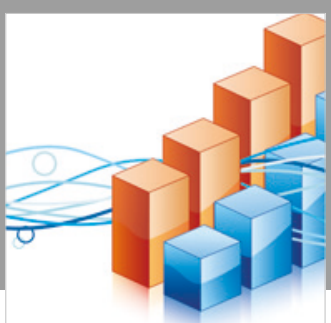

Advances in

Operations Research

\section{-n-m}
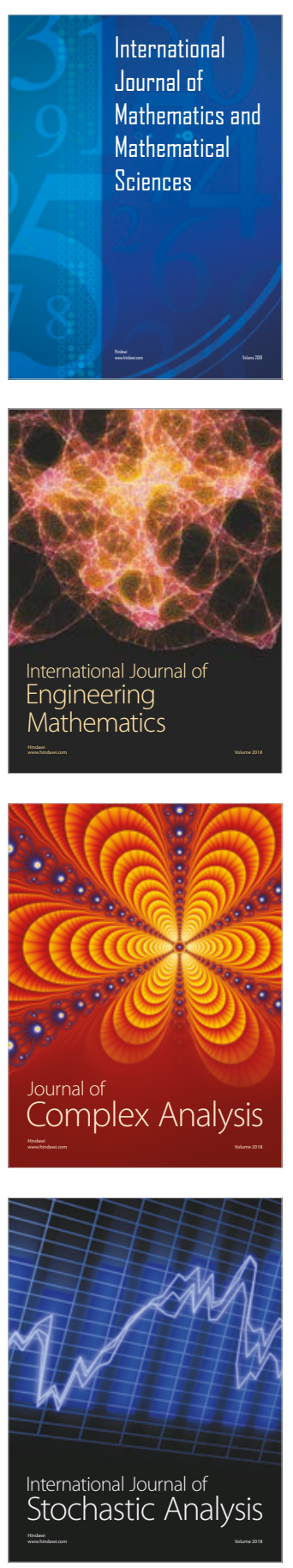
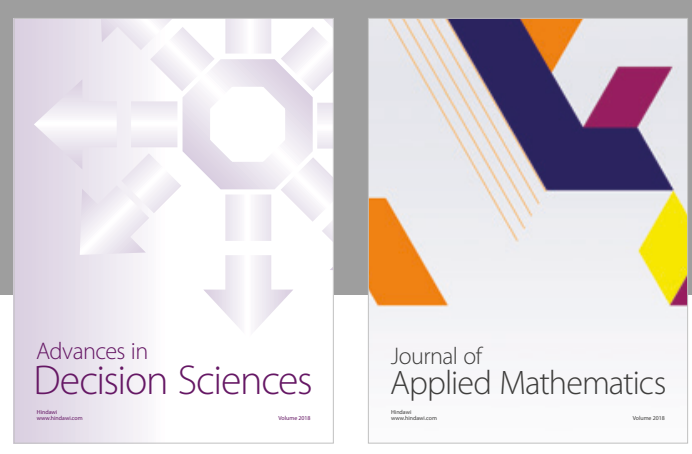

Journal of

Applied Mathematics
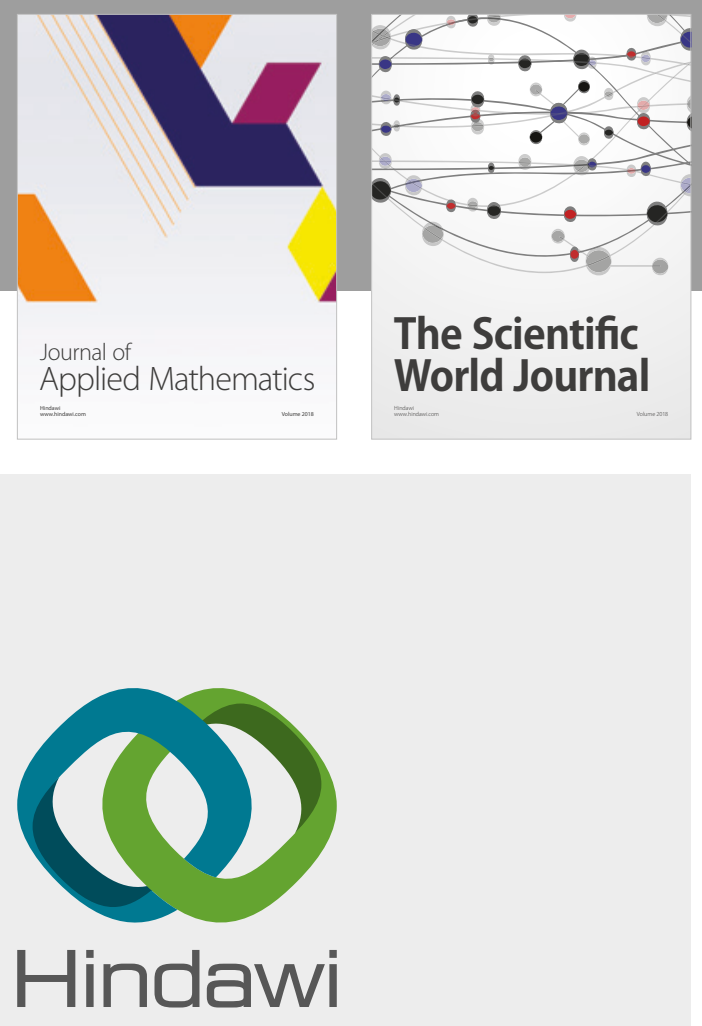

Submit your manuscripts at

www.hindawi.com

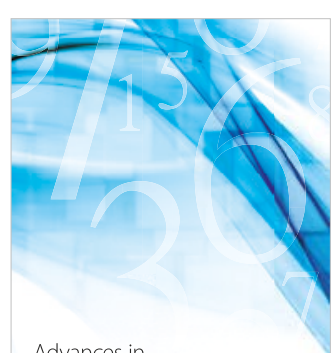

Advances in
Numerical Analysis
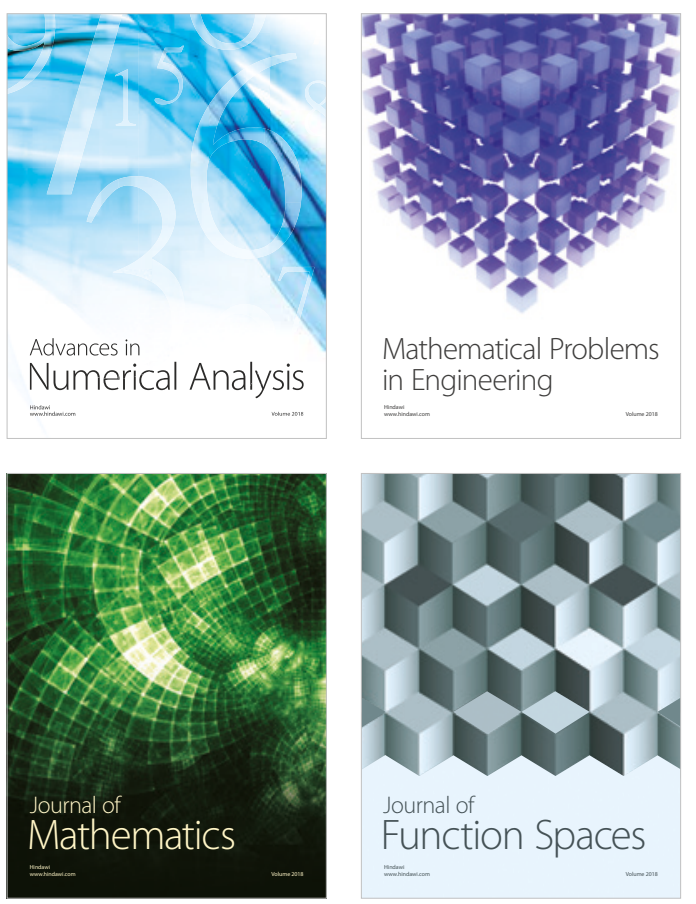

Mathematical Problems in Engineering

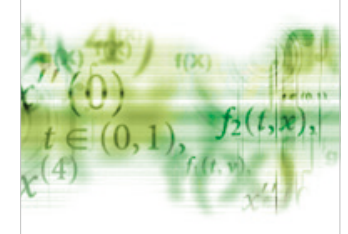

International Journal of

Differential Equations

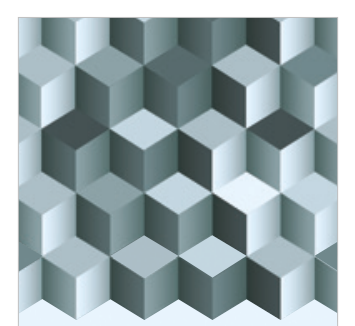

Journal of

Function Spaces

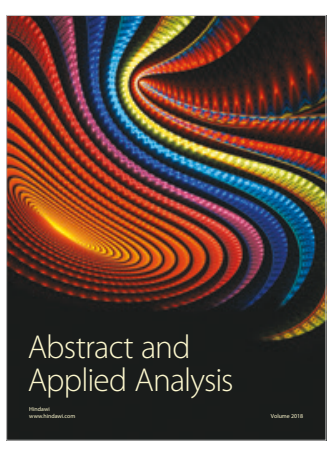

The Scientific

World Journal

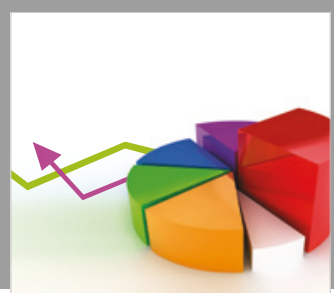

Journal of

Probability and Statistics
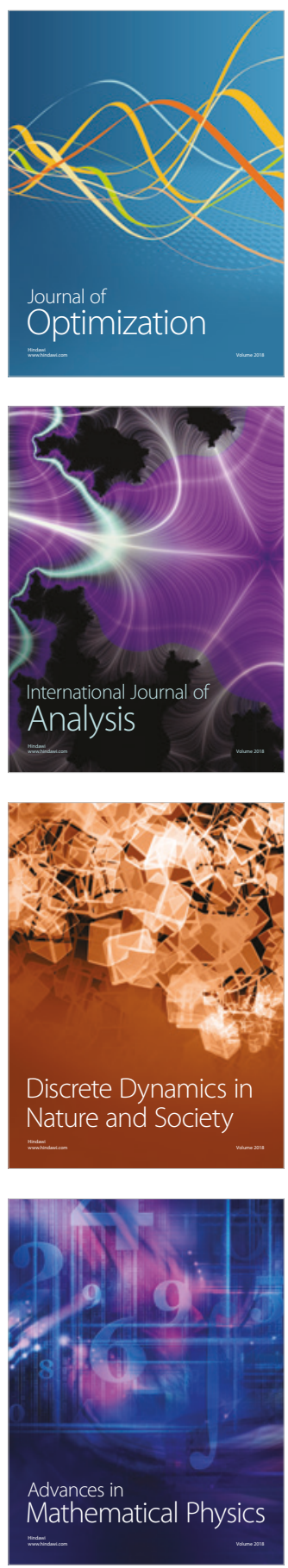